\title{
Drag Prediction for the DLR-F6 Wing/Body and DPW Wing Using CFL3D and OVERFLOW on an Overset Mesh
}

\author{
Anthony J. Sclafani; John C. Vassberg† Neal A. Harrison; Mark A. DeHaan ${ }^{\S}$ \\ The Boeing Company, Phantom Works, Huntington Beach, CA, 92647, USA \\ Christopher L. Rumsey, S. Melissa Rivers", Joseph H. Morrison** \\ NASA Langley Research Center, Hampton, VA, 23681, USA
}

\begin{abstract}
A series of overset grids was generated in response to the $3^{\text {rd }}$ AIAA CFD Drag Prediction Workshop (DPW-III) which preceded the $25^{\text {th }}$ Applied Aerodynamics Conference in June 2006. DPW-III focused on accurate drag prediction for wing/body and wing-alone configurations. The grid series built for each configuration consists of a coarse, medium, fine, and extra-fine mesh. The medium mesh is first constructed using the current state of best practices for overset grid generation. The medium mesh is then coarsened and enhanced by applying a factor of 1.5 to each $(\mathrm{I}, \mathrm{J}, \mathrm{K})$ dimension. The resulting set of parametrically equivalent grids increase in size by a factor of roughly 3.5 from one level to the next denser level. CFD simulations were performed on the overset grids using two different RANS flow solvers: CFL3D and OVERFLOW. The results were post-processed using Richardson extrapolation to approximate grid converged values of lift, drag, pitching moment, and angle-of-attack at the design condition. This technique appears to work well if the solution does not contain large regions of separated flow (similar to that seen in the DLR-F6 results) and appropriate grid densities are selected. The extra-fine grid data helped to establish asymptotic grid convergence for both the OVERFLOW FX2B wing/body results and the OVERFLOW DPW-W1/W2 wing-alone results. More CFL3D data is needed to establish grid convergence trends. The medium grid was utilized beyond the grid convergence study by running each configuration at several anglesof-attack so drag polars and lift/pitching moment curves could be evaluated. The alpha sweep results are used to compare data across configurations as well as across flow solvers. With the exception of the wing/body drag polar, the two codes compare well qualitatively showing consistent incremental trends and similar wing pressure comparisons.
\end{abstract}

\footnotetext{
*Aerodynamics Engineer 4, AIAA Member

$\dagger$ Aerodynamics Engineer 6, Technical Fellow, AIAA Associate Fellow

$\ddagger$ Aerodynamics Engineer 2, AIAA Member

$\S$ Aerodynamics Engineer 5

ISenior Research Scientist, AIAA Associate Fellow

"Research Scientist, AIAA Member

** Research Scientist, AIAA Senior Member
} 


\section{Nomenclature}

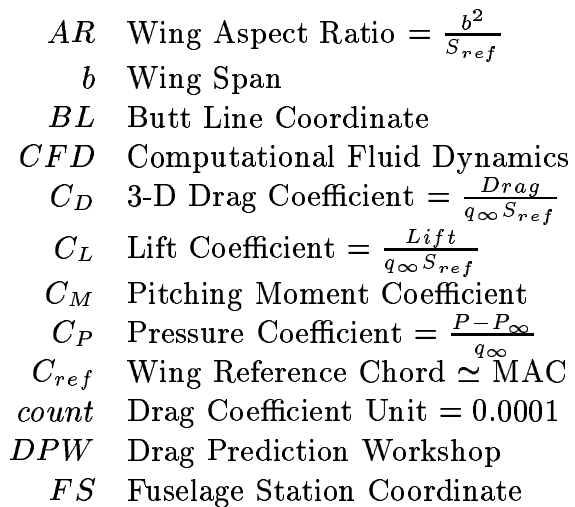

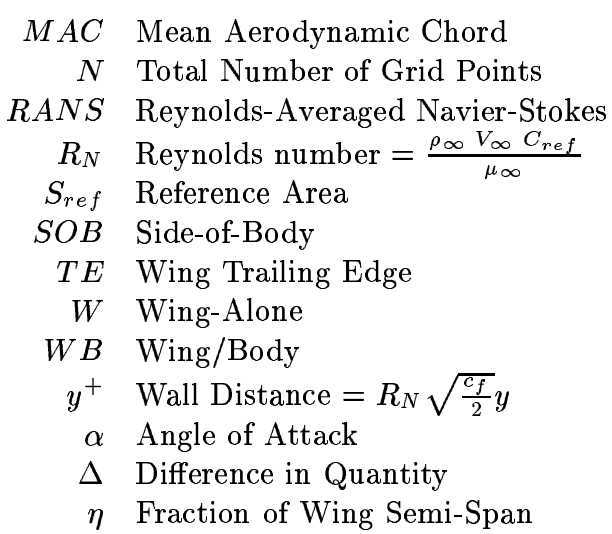

\section{Introduction}

The Drag Prediction Workshop (DPW) provides a forum where both users and developers of Computational Fluid Dynamics (CFD) programs designed to solve the Reynolds-averaged Navier-Stokes (RANS) equations can discuss issues related to overall CFD methods, including topics such as turbulence modeling and grid generation. The workshop focuses the discussion by attempting to gauge how well CFD can predict aerodynamic forces and moments for a given geometry. Drag is the primary metric used to evaluate results because of the importance accurate drag prediction has within an aircraft design environment.

The first workshop (DPW-I) took place in June of 2001. The geometry chosen for DPW-I was the DLR-F4 wing/body (WB) because it represents a typical transport configuration designed to cruise at transonic speeds and test data is publicly available. The level of participation for DPW-I was to a point where a meaningful statistical analysis could be performed on its set of CFD data. The results of the analysis were somewhat discouraging, showing a large spread in fixed- $C_{L}$ drag. ${ }^{1,2}$ Even though the data showed a lot of room for improvement, DPW-I was considered successful because it brought together CFD developers and practitioners and focused their efforts on a common problem. Ultimately, the findings from DPW-I reminded the CFD and applied aerodynamics communities that CFD is not yet a fully matured discipline. In addition, this workshop created a sustained interest in industry-relevant drag prediction which helped steer the DPW organizing committee to a direction of increased geometry complexity for the next workshop. For more information on DPW-I, see References 3 and 4.

The second workshop (DPW-II) was held in June of 2003. For this workshop, the DLR-F6 was chosen as the test case geometry for the same reasons as the F4. The F6 has nearly the same WB geometry as the F4 and experimental data is available to the public. Furthermore, the F6 was tested in a wing/body/nacelle/pylon configuration. One objective of DPW-II was nacelle/pylon incremental drag prediction. At the conclusion of the second workshop, it was clear that separated flow regions at the wing/body and wing/pylon junctures made it difficult to draw meaningful conclusions with respect to grid convergence and drag prediction. As a result, the organizing committee decided to take a step back in geometry complexity for the next workshop. For more information on DPW-II, see References 5 and 6.

The third workshop (DPW-III) was held in June of 2006. The F6 WB from DPW-II was reused as the baseline configuration for DPW-III creating a bridge between the two workshops. The primary goal of DPWIII was to revisit the WB grid convergence study with and without separated flow at the wing/body juncture. In order to meet this goal, a fairing was designed to eliminate the separation. Details of the fairing design can be found in Reference 7. The workshop committee also hoped to reduce or eliminate the wing trailing-edge separation observed in wind tunnel flow visualization images by increasing the Reynolds number for the test case. The changes in both geometry and flow condition made DPW-III a blind test of CFD methods because no test data would be available prior to the workshop. In addition to the F6 WB configuration, wing-alone geometries were created so workshop participants could analyze a simple configuration with no separation issues. The wing-alone test case also provided an opportunity to perform an extended grid convergence study where solutions from coarse, medium, fine, and extra-fine meshes could be evaluated.

This paper summarizes DPW-III results by comparing data from two different flow solvers (CFL3D and 
OVERFLOW) run on the same overset mesh. Given the higher $R_{N}$ flow condition and new geometry, minimal CFD-to-tunnel data comparisons will be made. For a comprehensive OVERFLOW-to-tunnel comparison of F6 WB data at 3 million $R_{N}$, see Reference 8 . The focus of this paper will be on the results of the grid convergence study and how well the two flow solvers compare. The WB fairing and wing-alone geometries will be discussed in Section II followed by some notes on grid generation in Section III. A complete review of the results is given in Section IV.

\section{Geometry Descriptions}

\section{II.A. DLR-F6 Wing/Body and FX2B Fairing}

The baseline WB configuration for DPW-III was the DLR-F6, which is the same geometry used as the test case in DPW-II. The F6 wind tunnel model represents an aircraft designed to cruise at transonic speeds with a wing leading-edge sweep of $27.1^{\circ}$ and a quarter-chord sweep of $25^{\circ}$. The dihedral of the wing is $4.787^{\circ}$. More reference quantities for this model are provided below.

$\begin{array}{rl}S_{r e f} / 2 & 72,700.0 \mathrm{~mm}^{2} \\ C_{r e f} & 141.2 \mathrm{~mm} \\ b / 2 & 585.647 \mathrm{~mm} \\ A R & 9.5(\simeq 9.436)\end{array}$

$\begin{array}{cl}X_{\text {ref }} & 157.9 \mathrm{~mm} \\ Y_{\text {ref }} & 0.0 \mathrm{~mm} \\ Z_{\text {ref }} & -33.92 \mathrm{~mm}\end{array}$

Note that an aspect ratio of 9.5 is used herein instead of the computed value. The purpose of this is to remain consistent with the open literature on this geometry. A planform view of the F6 WB configuration is shown in Figure 1. For more detailed information on this geometry, see References 5 and 9.

The DLR-F6 model has an acute angle between the fuselage and wing upper-surface at the trailing-edge. At roughly $60^{\circ}$, this relatively low angle contributes to flow separation at the WB juncture. A view from behind the model, as shown in Figure 2, best illustrates the problem area. To help alleviate this issue, a new WB fairing was developed by Vassberg ${ }^{7}$ with the goal of completely removing the separation bubble present at the design conditions. For consideration of a possible follow-on wind-tunnel test, the only design constraint for this fairing was that it does not cut into the baseline DLR-F6 geometry. Further, no attempt was made to optimize drag reduction. The fairing was designed in two parts. The first part represents a typical WB fairing seen on most low-wing transport aircraft. Designated FX1, this fairing is everywhere outside or tangent to the baseline fuselage and increases the fuselage-to-wing angle by about $20^{\circ}$. Figure 3 shows the rear view of FX1. To ensure fully attached WB juncture flow, a second bump-like fairing was developed. This second part to the overall fairing was designated FX2 and includes FX1 as the starting point. The FX2 fairing is shown from behind the model in Figure 4 and from above in Figure 5. The final fairing geometry was slightly modified during the CATIA surface generation process, hence the name FX2B.

\section{II.B. DPW Wings W1 and W2}

Two wing-alone geometries (DPW-W1/W2) were developed for DPW-III. Both have in common a simple trapezoidal planform shape with no leading-edge or trailing-edge breaks. Their leading-edge sweep is $17.2^{\circ}$ and they have a quarter-chord sweep of $15.0^{\circ}$. The following summarizes their reference quantities.

$\begin{array}{rl}S_{r e f} / 2 & 145,161.0 \mathrm{~mm}^{2} \\ C_{r e f} & 197.556 \mathrm{~mm} \\ b / 2 & 762.0 \mathrm{~mm} \\ A R & 8.0\end{array}$

$\begin{array}{cl} & W 1 \\ X_{\text {ref }} & 6847.145 \mathrm{~mm} \\ Y_{\text {ref }} & 0.0 \mathrm{~mm} \\ Z_{\text {ref }} & 2226.615 \mathrm{~mm}\end{array}$

$\begin{array}{cl} & W 2 \\ X_{\text {ref }} & 154.245 \mathrm{~mm} \\ Y_{\text {ref }} & 0.0 \mathrm{~mm} \\ Z_{\text {ref }} & 0.0 \mathrm{~mm}\end{array}$

The airfoil selected for DPW-W1 is representative of a supercritical section found on most modern transport aircraft. At the design condition of Mach 0.76 and $C_{L}=0.50$, the upper surface pressure distribution has a nearly flat, roof-top shape with a shock located at about $57 \% x / c$. The second wing, DPW-W2, was created by optimizing the first.

A Tranair single-point optimization at 0.76 Mach and 5 million Reynolds number was performed on the DPW-W1 wing to create a test for drag increment prediction. Since the goal was to preserve the planform and thickness of the original wing, only camber and twist variables were allowed. The variables were laid out at 7 
spanwise cuts, with 5 camber and 1 twist variable on each cut, for a total of 42 geometry degrees of freedom. The twist variable rotates the airfoil about its trailing edge in a plane orthogonal to the y-axis. In addition, the angle of attack was allowed to vary in order to maintain the same lift coefficient as the original wing.

No attempt was made to tune the locations of the geometry variables, so the camber locations were at every 0.2 in $x / c$, starting at 0.1 , and ending at $0.9 x / c$. These camber "bumps" are given by quintic B-splines which interpolate the amplitude of the central knot and vanish at neighboring end-point knot locations. The span cuts were at $0.0,0.2,0.4,0.6,0.75,0.90$, and 1.0 in fractions of semi-span. This allows the entire wing to change shape, including both the wing root and the wing tip. To prevent a singular problem definition, the twist variable at the wing root was fixed at 0 . All variables express a delta change in geometry location from the initial wing.

The optimization used the variables described above to minimize the drag as computed by the farfield formulation of induced, profile and wave drags. This was subject to the fixed lift constraint and a set of transpiration modeling constraints which are necessary to keep the optimization step size within the domain of applicability of the model problem. The optimizer used was the sequential quadratic programming algorithm, NPSOL. The largest camber change was approximately $1.4 \mathrm{~mm}$ in full scale units, with the twist change being between $\pm 0.5^{\circ}$.

Additional information regarding the design of these wings is provided by Zickhur and Tinoco ${ }^{10}$ in the workshop presentations on geometry review.

\section{Overset Grid Generation}

The overset grids for the DLR-F6 wing/body and DPW-W1/W2 wing were built by the Boeing authors. As with previous workshops, the medium grid was first generated using tools and techniques that are in-line with the current state of best practices for overset grid generation. ${ }^{11,12}$ The medium grid, which is considered typical for drag-quality design studies, was then modified by removing points for the coarser grid and adding points for the finer grid. Each grid index increased by a factor of 1.5 from one grid size to the next. In all, four grid families were constructed with each family consisting of a coarse, medium, fine, and extra-fine grid. Two grid families belong to the wing/body study and two belong to the wing-alone study. An attempt was made to construct the grids in a way that maintains surface breaks through at least three multigrid levels.

\section{III.A. DLR-F6 Wing/Body Grid}

The overset grid generation process typically begins with the extraction of points from trimmed surfaces followed by surface grid generation. Since the DPW-II F6 WB geometry was carried over to DPW-III, these first two steps were already completed. The baseline F6 medium surface grid from DPW-II was the starting point for the WB grid generation process. It consisted of 12 zones - 9 surface abutting grids and 3 box grids. Figure 6 shows the WB surface grid layout with the wing modeled by three c-mesh grids, two trailing-edge cap grids, and a wingtip cap grid. The body also has cap grids at the nose and tail. The volume grids were regenerated to ensure adequate grid point number and spacing in the boundary layer for the higher Reynolds number flow specified for DPW-III. References 13, 14, and 15 represent some of the tools used to build-up the volume grids. Some basic grid information for the baseline F6 WB grid family is provided in Table 1.

\begin{tabular}{|c|c|c|c|c|c|}
\hline Grid & Points & $1^{\text {st }}$ Cell Size & $y^{+}$ & Const Cells & Growth Rate \\
\hline \hline Coarse & $2,387,918$ & $.00055 \mathrm{~mm}$ & .90 & 2 & 1.29 \\
\hline Medium & $7,985,236$ & $.00038 \mathrm{~mm}$ & .62 & 3 & 1.19 \\
\hline Fine & $26,892,352$ & $.00025 \mathrm{~mm}$ & .41 & 4 & 1.12 \\
\hline Extra-Fine & $82,584,096$ & $.00019 \mathrm{~mm}$ & .31 & 6 & 1.09 \\
\hline
\end{tabular}

Table 1: Grid Information for the DLR-F6 Wing/Body Baseline Configuration.

Following the gridding guidelines provided by the DPW-III committee, the minimum $y^{+}$spacing for the coarse grid was set below 1.0 and the total grid size grew by a factor of roughly 3 from one grid level to the next. The number of constantly-spaced cells at the surface was two for the coarse grid and increased with grid size. Views of the volume grid at the symmetry plane are provided in Figures 7, 8, and 9. In Figure 7 the global box grid, which extends beyond the frame of the image to the far-field, is shown in blue. The mid-field box grid for the body is shown in red, and the body grid is black. Figures 8 and 9 show details of the nose and 
tail patches used to avoid grid singularities in the body grid. Figure 10 shows the surface abutting volume grids of the wing and body as well as the mid-field box grids. Finally, Figures 11 and 12 provide details of the wingtip and wing trailing-edge cap grids.

The FX2B medium grid was constructed by modifying some of the baseline F6 grids. The body, wing/body collar, and wing trailing-edge cap grids were updated to accurately model the FX2B fairing. More surface points were needed to capture the fairing's highly nonlinear bump shape which is characterized by a small radius in the crown-to-keel direction (see Figure 4). This increase in surface grid points can be seen in the total grid point count summarized in Table 2.

\begin{tabular}{|c|c|c|c|c|c|}
\hline Grid & Points & $1^{\text {st }}$ Cell Size & $y^{+}$ & Const Cells & Growth Rate \\
\hline \hline Coarse & $2,395,170$ & $.00055 \mathrm{~mm}$ & .90 & 2 & 1.29 \\
\hline Medium & $8,020,348$ & $.00038 \mathrm{~mm}$ & .62 & 3 & 1.19 \\
\hline Fine & $26,969,192$ & $.00025 \mathrm{~mm}$ & .41 & 4 & 1.12 \\
\hline Extra-Fine & $82,805,148$ & $.00019 \mathrm{~mm}$ & .31 & 6 & 1.09 \\
\hline
\end{tabular}

Table 2: Grid Information for the DLR-F6 Wing/Body FX2B Configuration.

The same process was used to build-up both baseline and FX2B volume grids, so the grid parameters summarized in Tables 1 and 2 are the same except for number of points.

\section{III.B. DPW-W1/W2 Wing-Alone Grid}

The definition of the wing-alone geometry was provided in an IGES file, so the complete grid generation process as described in Reference 11 was employed. The surface grid layout is shown in Figure 13. For this simple wing-alone configuration, the surface is represented by three overset grids: a c-mesh for the wing (blue), a wrap-around trailing-edge cap grid (green), and another wrap-around grid for the wingtip (red). These three surface abutting grids are combined with two box grids to make-up the overall grid system. Parameters for the complete grid family of $\mathrm{W} 1$ and $\mathrm{W} 2$ are provided in Table 3.

\begin{tabular}{|c|c|c|c|c|c|}
\hline Grid & Points & $1^{\text {st }}$ Cell Size & $y^{+}$ & Const Cells & Growth Rate \\
\hline \hline Coarse & $1,442,285$ & $.00055 \mathrm{~mm}$ & .90 & 2 & 1.29 \\
\hline Medium & $4,856,149$ & $.00038 \mathrm{~mm}$ & .62 & 3 & 1.19 \\
\hline Fine & $16,265,909$ & $.00025 \mathrm{~mm}$ & .41 & 4 & 1.12 \\
\hline Extra-Fine & $55,014,321$ & $.00019 \mathrm{~mm}$ & .31 & 6 & 1.09 \\
\hline
\end{tabular}

Table 3: Grid Information for the DPW-W1/W2 Wing-Alone Configuration.

The volume grid layout is similar to the wing/body and can be seen in Figures 14 and 15. The box grid extending to the far field is partially shown in Figure 14 (red) and not shown at all in Figure 15.

\section{Results}

DPW-III participants were required to provide results on one of two test cases - the wing/body (Case 1) or the wing-alone (Case 2). At the time of the workshop, overset structured grid data were generated using OVERFLOW for both test cases and CFL3D for Case 1. In an attempt to expand the overset database, additional CFL3D runs were made on Case 2 grids. All CFL3D data presented in this paper were provided by the NASA Langley Research Center (LaRC). The majority of OVERFLOW data was provided by Boeing Phantom Works in Huntington Beach, CA. LaRC attempted to run OVERFLOW for Case 1 using Menter's SST turbulence model but code related problems hindered full completion of this effort. The freestream flow conditions used for both test cases are given in Table 4.

\begin{tabular}{|c|c|c|c|}
\hline Test Case & $R_{N}$ (based on $C_{\text {ref }}$ ) & Mach & Temperature \\
\hline \hline wing/body & $5 \times 10^{6}$ & 0.75 & $580^{\circ} \mathrm{R}$ \\
\hline wing-alone & $5 \times 10^{6}$ & 0.76 & $580^{\circ} \mathrm{R}$ \\
\hline
\end{tabular}

Table 4: Flow Conditions. 
Workshop committee guidelines called for a fully turbulent boundary layer (no specified transition) and a free-air simulation, so wind tunnel walls or the model support system were not included. The discussion of results will focus on the single point grid convergence study for both cases. Where enough CFD data are available, CFL3D-to-OVERFLOW comparisons are made.

\section{IV.A. Flow Solver and Computing Platform}

Some general information on the flow solvers used to generate overset grid results will be given, and the computing platform used to run each code is briefly discussed.

\section{IV.A.1. CFL3D}

CFL3D ${ }^{16,17}$ was developed at the NASA Langley Research Center as a cell-based RANS code for structured grids. It is a general purpose 2D/3D solver capable of simulating steady or unsteady flows. The boundary layer can be modeled as laminar or turbulent with a choice of several different turbulence models. The DPW-III test cases were run in both thin-layer and full Navier-Stokes modes with the upwind Roe scheme and Menter's $\mathrm{SST}^{18}$ turbulence model.

CFL3D version 6 was run in parallel using 64-bit precision on Columbia which is a 10,240 CPU SGI Altix supercluster at NAS. The wing/body fine grid (27M points) in full Navier-Stokes mode required 150 hours of wall-clock time to complete 2000 cycles on 10 processors. Anywhere from 2000 to 5000 cycles were needed to reach full convergence, so some cases took as long as 375 hours (almost 16 days) to finish. This relatively slow turn-around is partially due to the code's inability to perform mesh sequencing on overset grids and the fact that grids cannot be split for improved parallel performance. CFL3D performs significantly better on multiple block grid topologies using 1-1 blocking.

\section{IV.A.2. OVERFLOW}

OVERFLOW ${ }^{19}$ was developed by NASA with numerous contributions from academia and industry. It is a node-based RANS code specifically designed for structured overset grids. Like CFL3D, it is a general purpose 2D/3D solver capable of simulating steady or unsteady flows. Included in the options for turbulence modelling are the one-equation Spalart-Allmaras (SA) ${ }^{20}$ and the two-equation Menter SST. The Boeing Phantom Works team chose to run OVERFLOW in a full Navier-Stokes mode with the Roe upwind scheme and SA turbulence model to be consistent with their DPW-II data. ${ }^{8}$ A limited amount of results will be presented from thinlayer/SA and full Navier-Stokes/SST runs. The full Navier-Stokes/SST runs were made by the NASA LaRC authors.

Message Passing Interface (MPI) version 2.0z of OVERFLOW was run for this study using 64-bit precision and grid sequencing to accelerate convergence. The parallel processing was performed on a Linux PC cluster which consists of 906 Opteron dual CPU nodes with 4 GB of memory each. The wing/body fine grid (27M points) in full Navier-Stokes mode required 9 hours of wall-clock time to complete 1000 cycles on 22 processors. Full convergence was achieved after 4000 cycles, so roughly 36 hours was needed for each wing/body fine grid case.

\section{IV.B. DLR-F6 Wing/Body Results}

The wing/body results are presented in four parts. The first part focuses on force and moment results from the grid convergence study. This discussion is followed by a look at wing pressures, which is intended to help explain some of the drag data trends observed in the grid study. Side-of-body and trailing-edge streamline images will also be presented in this second part. Drag polars and lift/pitching moment curves are discussed in the third part of the results section, and the fourth part addresses an OVERFLOW side-study performed on the F6 medium grid.

\section{IV.B.1. DLR-F6 Grid Convergence Study}

The primary objective of the grid convergence study is to approximate grid-converged values for forces and moments. In other words, this study will estimate the absolute level of drag if an infinite number of grid points are applied. Richardson extrapolation is used to evaluate the CFD data by plotting them as a function of $N^{-2 / 3}$, where $N$ is the total number of points for each mesh. The $2 / 3$ power is based on the order of 
accuracy (second-order) of the numerical method used to compute the results. This application of Richardson extrapolation only works if the CFD dataset is from a parametrically-equivalent grid family where the coarse mesh is a subset of the next denser mesh. Reference 21 provides details on the importance of uniformly refined grids for meaningful convergence studies. If this criteria is met along with some other basic requirements such as converging all solutions to the same level and running at constant $C_{L}$ for drag studies, the quality of the results can be measured by how linear they are when plotted against $N^{-2 / 3}$. Asymptotic grid convergence is achieved if a straight line can be drawn through the data.

For the grid convergence study, the wing/body test case was run fully turbulent at a constant $C_{L}$ of 0.5 . The CFL3D and OVERFLOW total drag results from the full Navier-Stokes runs are shown in Figure 16. In this plot, baseline F6 data are colored red and FX2B data are blue. The OVERFLOW-SA data include results from an extra-fine grid consisting of roughly $83 \mathrm{M}$ points. Since the coarse grid drag results consistently break-away from the linear trend line projected from the medium and fine grid data, an extra-fine grid is needed to help clarify whether asymptotic grid convergence is achieved. Since this extremely dense grid was built well after the workshop, CFL3D results could not be produced in time for this paper. Linear extrapolation was used to estimate the level of total drag obtained when the number of grid points goes to infinity. The arrows shown in Figure 16 point to the extrapolated values which are summarized in Table 5.

\begin{tabular}{|c|c|c|c|c|}
\hline Source & Extrapolated Grids & $F 6($ cnts $)$ & $F X 2 B($ cnts $)$ & $\Delta($ cnts $)$ \\
\hline \hline CFL3D-SST & medium/fine & 274.7 & 265.6 & -9.1 \\
\hline OVERFLOW-SA & medium/fine & 276.0 & 259.6 & -16.4 \\
\hline OVERFLOW-SA & fine/extra-fine & 278.0 & 259.8 & -18.2 \\
\hline
\end{tabular}

Table 5: Wing/Body Total Drag Extrapolated to the Continuum, $\Delta=(F X 2 B-F 6)$.

When compared to the OVERFLOW-SA results, CFL3D-SST gives less total drag for the F6 and more for the FX2B, making the grid-converged increment between the two configurations significantly less (-9.1 cnts vs $-16.4 \mathrm{cnts})$. The OVERFLOW-SA results show a nearly linear trend for the FX2B medium, fine, and extra-fine grid data, which is highlighted in Table 5 by the small difference in drag between the medium/fine grid-converged value $(259.6 \mathrm{cnts})$ and the fine/extra-fine grid-converged value $(259.8 \mathrm{cnts})$. This indicates asymptotic grid convergence is essentially achieved. On the other hand, the F6 OVERFLOW-SA results do not follow a linear trend as shown in Table 5 by the 2 count difference between medium/fine and fine/extrafine grid-converged values. Running OVERFLOW in full Navier-Stokes mode with the SA turbulence model produces results that are not in the asymptotic range of convergence for the baseline F6 configuration. This conclusion is not surprising since the F6 geometry is characterized by a large side-of-body (SOB) separation bubble. Unfortunately, the FX2B drag increment from the medium grid data is 8 counts less than the gridconverged drag increment. This large error is thought to be caused by the SOB separation present in the baseline solution. Figure 16 includes OVERFLOW-SST data for the coarse and medium F6 grids. Compared to the SA results, the SST model gives about 3.5 counts more total drag for both grids.

The full Navier-Stokes pressure and skin friction drag components are plotted in Figures 17 and 18, respectively. It is important to look at the drag components for the grid convergence study to rule-out the possibility of cancelling errors. The level of pressure drag from the various solutions, relative to each other, is similar to the total drag. In other words, the CFL3D-SST F6 fine grid pressure drag is a little lower than OVERFLOW-SA and the same can be said for the total drag. The general trends in the pressure drag data are also consistent with the total drag. The skin friction drag data, shown in Figure 18, is considerably less sensitive to grid refinement than pressure drag (note the tighter scale used for the skin friction plot). The variation in OVERFLOW-SA skin friction going from coarse to extra-fine is less than 0.5 counts for the F6 results and less than 2 counts for FX2B. Both codes predict higher skin friction for the FX2B configuration with a 2 count increment (FX2B-F6) for the medium grid. Finally, the F6 OVERFLOW results show the SA model computes about 1.5 counts more skin friction drag than the SST model on the medium grid.

Pitching moment results from the full Navier-Stokes solutions are summarized in Figure 19. Both CFL3D and OVERFLOW compute a more nose-down moment for FX2B at a $C_{L}$ of 0.5 . The F6 results from OVERFLOWSA show a more linear trend than the FX2B results which is opposite of the total drag data shown in Figure 16. Angle-of-attack data is presented in Figure 20. This plot shows both codes agree on the direction of the alpha change to hold $C_{L}$ going from F6 to FX2B. As with drag and pitching moment, it is difficult to comment on whether or not the CFL3D results have reached asymptotic grid convergence using the coarse/medium/fine data. When the extra-fine grid data point is added to the OVERFLOW-SA results, it is easier to see that asymptotic grid convergence is achieved for angle-of-attack. 
The NASA LaRC authors conducted a side study by running CFL3D in both thin-layer and full Navier-Stokes modes. The expression "full Navier-Stokes" means the viscous flux terms are computed in all three directions including cross terms and "thin-layer" means only the terms in the viscous thin-layer (i.e., boundary layer) are computed. For comparison purposes, thin-layer OVERFLOW-SA data were generated for the medium grid only. The results are summarized in Figure 21. F6 data is plotted in Figure 21(a) and FX2B data is in Figure 21(b). The two solvers agree: a thin-layer simulation yields less drag for both configurations. Table 6 lists medium grid drag increments for the thin-layer effect.

\begin{tabular}{|c|c|c|}
\hline Source & $F 6 \Delta C_{D}$ & $F X 2 B \Delta C_{D}$ \\
\hline \hline CFL3D-SST & $3.1 \mathrm{cnts}$ & $1.0 \mathrm{cnts}$ \\
\hline OVERFLOW-SA & $7.8 \mathrm{cnts}$ & $1.1 \mathrm{cnts}$ \\
\hline
\end{tabular}

Table 6: Thin-Layer Effect on Medium Grid Drag Results: $\Delta=($ full - thin $)$.

The magnitude of the F6 drag increment is significantly larger than FX2B, once again highlighting the fundamental difference in the flowfield - the F6 SOB separation. Figure 22 compares surface streamlines on the wing upper surface at the SOB. The separation bubble is smaller in the thin-layer solution which increases the inboard wing loading allowing the angle-of-attack to drop for constant $C_{L}$. The alpha reduction coupled with the pressure drag reduction from a smaller separation bubble results in lower total drag. Although not shown, the CFL3D surface streamline comparison looks similar to the OVERFLOW images in Figure 22. The thin-layer CFL3D-SST results for the coarse, medium, and fine grids are shown in Figure 23. The arrows point to the medium/fine grid-converged values for total drag which give an increment of -5.1 counts compared to the full Navier-Stokes grid-converged drag increment of -9.1 counts (see Table 5).

\section{IV.B.2. DLR-F6 Wing Pressures and Streamlines}

Figures 24, 25, and 26 contain F6 wing pressure comparisons at semispan locations of $0.150,0.332$, and 0.637, respectively. Each figure shows the coarse/medium/fine/extra-fine grid OVERFLOW pressures on the left and the coarse/medium/fine grid CFL3D pressures on the right. All pressures are from the grid study runs which were made at a $C_{L}$ of 0.5 . The 0.150 comparison in Figure 24 cuts through the separation bubble present on the F6 wing. This can be seen in the upper-surface pressures as recovery to free-stream is not achieved. The general character of the $C_{p}$ distribution is the same between codes with a minimum level of roughly $-0.9 C_{p}$ occuring at about the same $\mathrm{x} / \mathrm{c}$ location. Figure 25 compares pressures where the shock is strong and well defined. It is a good illustration of how grid refinement affects the computed shock location and strength. The OVERFLOW data show a significant difference in shock definition going from the coarse grid to the medium whereas the medium/fine/extra-fine grid pressure data are tightly grouped showing minor variation in shock location. This observation helps explain why the coarse grid drag data tend to be outside the asymptotic range of grid convergence. Although, it is unclear why the coarse grid drag is consistently higher than the denser grids; a weaker shock will produce less wave drag. In order to fully understand the trend of lower drag with increasing grid points, a more detailed drag breakdown should be made. The CFL3D pressure data shown on the right of Figure 25 give a similar story. The coarse grid shock definition is considerably different than the medium and fine grid results. The two codes differ in that the OVERFLOW shock tends to "stand-up" with grid refinement but not move very much in $\mathrm{x} / \mathrm{c}$. The CFL3D shock also "stands-up" with grid refinement but, unlike OVERFLOW, it moves forward as well. The forward movement brings the CFL3D shock location more in-line with OVERFLOW. Both codes put the fine grid shock at about $0.24 \mathrm{x} / \mathrm{c}$ at this wing station. The pressure comparisons shown in Figure 26 tell a similar story. The coarse grid $C_{p}$ distributions are significantly different compared to the denser grid results for both codes, and there is general agreement across solvers for the medium and fine grid pressures.

OVERFLOW surface streamlines from the medium grid solutions at a $C_{L}$ of 0.5 are compared in Figures 27 and 28. Figure 27 compares the wing/body juncture flow at the upper-surface wing trailing-edge. The DLR-F6 separation bubble seen in Figure 27(a) is completely eliminated by the FX2B fairing as shown in Figure 27(b). The size of the separation bubble is quantified in Figure 22 for the medium grid full NavierStokes solution. To see if the bubble size changes with grid refinement, the same two dimensions called-out in Figure 22 are tabulated below. The Butt Line (BL) coordinate is measured along the span of the wing and the Fuselage Station (FS) coordinate is measured along the fuselage longitudinal axis. 


\begin{tabular}{|c|c|c|}
\hline Grid & $B L(\mathrm{~mm})$ & $F S(\mathrm{~mm})$ \\
\hline \hline coarse & 95.5 & 170.6 \\
\hline medium & 102.7 & 153.2 \\
\hline fine & 107.6 & 143.9 \\
\hline extra-fine & 109.6 & 139.5 \\
\hline
\end{tabular}

Table 7: DLR-F6 OVERFLOW-SA Full Navier-Stokes Separation Bubble Size.

The dimensions in Table 7 show the separation bubble gets larger with increasing grid density. Computed bubble size was compared against flow visualization from ONERA for DPW-II. ${ }^{8}$ The spanwise extent (BL) of the separation bubble observed in the wind tunnel was scaled off a flow-viz image and found to be roughly $91 \mathrm{~mm}$. Every DLR-F6 medium grid OVERFLOW solution generated to date (including both DPW-II and DPW-III) computes a SOB separation bubble size larger than that observed in the ONERA test. Furthermore, running in full Navier-Stokes mode and refining the grid tends to make the bubble even larger.

Wing planform views comparing upper-surface streamlines are given in Figure 28. These are medium grid OVERFLOW-SA solutions at a $C_{L}$ of 0.5 . The streamline traces show spanwise flow at the trailing-edge but the boundary layer appears to be attached. For the 5 million Reynolds number OVERFLOW and CFL3D solutions, no trailing-edge separation was present on either the F6 or FX2B wings for the medium and fine grids.

\section{IV.B.3. DLR-F6 Drag Polars and Lift/Pitching Moment Curves}

All data presented in this sub-section are from full Navier-Stokes solutions where the medium grid was run at several alphas to establish force and moment trends.

The baseline F6 drag polar is shown in Figure 29. This plot compares OVERFLOW results using two different turbulence models and CFL3D/OVERFLOW results using the same turbulence model. The OVERFLOWSST polar is a rotation of the OVERFLOW-SA polar with a couple of counts more drag at low $C_{L}$ 's and rougly 7 counts more at high $C_{L}$ 's. The CFL3D-SST polar is not as smooth as OVERFLOW. The two sets of SST results agree well through the mid $C_{L}$ 's but there's a considerable difference at the low and high-end of the polar.

Before discussing the next set of drag polars, a couple of comments must be made regarding the FX2B data submitted to DPW-III.

- A full set of OVERFLOW-SST data could not be obtained for the FX2B configuration due to code-related problems, so alpha sweep data will not be presented for this code/configuration combination.

- The CFL3D FX2B alpha sweep data was found to be inconsistent with the single-point grid study data. The results presented in this section are different (and considered more correct) than what was submitted to the workshop. More information on this subject is provided in the Appendix.

FX2B drag polars are compared in Figure 30. As with the F6 drag polar comparison, the FX2B OVERFLOWSA results look smoother (less wavy) than CFL3D-SST.

Figure 31 provides a drag polar comparison between configurations with OVERFLOW-SA results plotted in Figure 31(a) and CFL3D-SST results plotted in Figure 31(b). The OVERFLOW comparison shows a drag improvement for the FX2B configuration throughout the $C_{L}$ range analyzed. The CFL3D results also show a drag improvement associated with the FX2B fairing but only through the mid- $C_{L}$ range.

A lift curve comparison for the baseline F6 configuration is shown in Figure 32. The OVERFLOW-SST curve is shifted to the right relative to OVERFLOW-SA. The OVERFLOW-SST simulation requires about $0.2^{\circ}$ more alpha than OVERFLOW-SA to hold $C_{L}$. The CFL3D-SST lift curve is nearly identical to OVERFLOW-SST at alphas below $0^{\circ}$. Above this alpha, the CFL3D-SST lift curve slope increases resulting in a nonlinear trend. The FX2B lift curve comparison is given in Figure 33. As with the F6 data, the OVERFLOW lift curve has a more consistent slope than CFL3D at higher alphas. Finally, Figure 34 compares lift curves between configurations for both OVERFLOW and CFL3D. The two codes are in general agreement. They show very little difference between the F6 and FX2B at low alphas. As the angle-of-attack increases, FX2B produces more lift as indicated by an increase in lift curve slope relative to F6.

F6 pitching moment curves are presented in Figure 35. All three sets of data look similar with a pitch break occuring near the design $C_{L}$ of 0.5 . The FX2B pitching moment comparison is given in Figure 36 followed by 
the configuration comparison for OVERFLOW and CFL3D in Figure 37. OVERFLOW shows the same $C_{M}$ for both configurations at a $C_{L}$ of about 0.14 while the CFL3D data meet at a higher $C_{L}$ of about 0.32 .

\section{IV.B.4. DLR-F6 Trailing-Edge Grid Study}

An OVERFLOW side-study (motivated by a prior study by Mavriplis ${ }^{22}$ ) was performed on the baseline DLRF6 geometry. The purpose of the study was to investigate the effect of enhancing the wing trailing-edge (TE) cap grid (see Figures 6 and 12) in the spanwise direction only. In particular, this was done to investigate the grid effect on TE flow behavior. For DPW-II, many unstructured-grid flow solvers predicted TE separation, but most structured-grid flow solvers (including CFL3D and OVERFLOW) did not. The goal was to make the surface grid cell aspect ratio as close to one as possible across the trailing-edge base. This is illustrated in Figure 38(b) where the enhanced inboard TE cap grid (yellow) is compared to the original full-wing TE cap grid (blue). The image in Figure 38(a) shows where these two TE cap grids meet close to the SOB. In order to meet the goal of a TE base cell AR of one, the number of spanwise points on the inboard TE cap grid increased by a factor of 4.5 and the full-wing TE cap grid spanwise point count increased by a factor of 22. The wing c-mesh grids were not modified.

The TE cap grid enhancement was made on the F6 medium grid and run at the flow condition given in Table 4. The run was started from scratch at the same angle-of-attack as the original grid, then restarted to target the design $C_{L}$ of 0.5 . The results show a minimal effect. The total drag for the dense TE cap grid solution increased by 0.4 counts at a $C_{L}$ of 0.5 . For the same angle-of-attack, $C_{L}$ increased by 0.004 . There was no significant change to the wing/body juncture separation bubble, and no wing TE separation was observed in either the original grid or the enhanced TE cap grid solutions. This result contrasts that of Mavriplis ${ }^{22}$ where a similar type of aspect ratio difference in two unstructured grids produced a significant decrease in lift for the same angle-of-attack.

\section{IV.C. DPW-W1/W2 Wing-Alone Results}

The wing-alone results are presented in three parts. The force and moment results from the grid convergence study are summarized in the first part. The second part is a brief look at wing pressures related to the grid study. Drag polars and lift/pitching moment curves are discussed in the third part as well as a description of upper-surface separation development for both W1 and W2.

\section{IV.C.1. $\quad$ DPW-W1/W2 Grid Convergence Study}

Two sets of data are presented for the wing-alone grid convergence study: constant angle-of-attack and constant $C_{L}$. The results from the constant angle-of-attack runs made for the workshop were informative but the need for constant $C_{L}$ data was apparent. After the workshop, the coarse/medium/fine/extra-fine W1 and W2 grids were run in OVERFLOW at a constant $C_{L}$ of 0.5. New CFL3D results were also generated after the workshop to enhance the overset grid database. For clarity, the constant alpha and constant $C_{L}$ data plots will be presented side-by-side when appropriate. All data presented here are from full Navier-Stokes simulations.

Total drag is plotted against grid size in Figure 39. Note that the same scale is used on both the constant alpha plot in Figure 39(a) and the constant $C_{L}$ plot in Figure 39(b). When analyzed at the same angle-ofattack, the OVERFLOW-SA data show the optimized W2 wing has more drag than W1. The W2 wing does have a significant drag improvement at $0.5 C_{L}$ as indicated in Figure 39(b). This is a good example of why drag studies are typically performed at constant $C_{L}$ conditions. The data presented in Figure 39 suggest the extra-fine grid results help establish asymptotic grid convergence for this simple wing-alone geometry. The coarse grid data tends to fall further away from the asymptote, particularly for the constant angle-of-attack drag trends. This behavior is consistent with the results of the wing/body grid convergence study.

Unfortunately, adequate time and resources were not available to obtain a full set of CFL3D data for the wing-alone test case. The W1 coarse/medium/fine grids were analyzed at constant alpha and only the $\mathrm{W} 1$ coarse/medium grids were analyzed at constant $C_{L}$. No CFL3D W2 cases were run. The CFL3D W1 medium/fine grid data are extrapolated to the continuum along with the OVERFLOW data at constant alpha. The extrapolated total drag data is summarized in Table 8. 


\begin{tabular}{|c|c|c|c|}
\hline Source & Extrapolated Grids & $W 1($ cnts $)$ & W2 (cnts) \\
\hline \hline CFL3D-SST & medium/fine & 198.9 & n/a \\
\hline OVERFLOW-SA & medium/fine & 201.7 & 203.6 \\
\hline OVERFLOW-SA & fine/extra-fine & 202.1 & 204.3 \\
\hline
\end{tabular}

Table 8: Wing-Alone Total Drag at Constant Alpha Extrapolated to the Continuum.

Based on these results, CFL3D-SST predicts roughly 3 counts less drag for W1 than OVERFLOW-SA at $0.5^{\circ}$ angle-of-attack. The results in Table 8 also show how the medium/fine/extra-fine grid data follow a nearly linear trend with grid refinement with less than a 1 count drag difference at the continuum between the medium/fine and fine/extra-fine extrapolated data. The same extrapolation is made on the constant $C_{L}$ OVERFLOW-SA results plotted in Figure 39(b).

\begin{tabular}{|c|c|c|c|}
\hline Source & Extrapolated Grids & W1 (cnts) & W2 (cnts) \\
\hline \hline OVERFLOW-SA & medium/fine & 213.8 & 202.5 \\
\hline OVERFLOW-SA & fine/extra-fine & 213.7 & 203.2 \\
\hline
\end{tabular}

Table 9: Wing-Alone Total Drag at Constant $C_{L}$ Extrapolated to the Continuum.

Table 9 does not include CFL3D results because the fine and extra-fine grids were not analyzed at constant $C_{L}$. Once again, asymptotic grid convergence is demonstrated in the OVERFLOW-SA results with only a 0.1 count difference in the W1 constant $C_{L}$ extrapolated data and a 0.7 count difference in the W2 data. Using the fine/extra-fine grid extrapolated total drag levels, the wing optimization produces a 10.5 count drag improvement at a $C_{L}$ of 0.5 .

The grid converged pressure drag data are presented in Figure 40 with constant alpha results in Figure 40(a) and constant $C_{L}$ in Figure 40(b). In general, the pressure drag trends with grid refinement are very similar to the total drag results. This leads one to believe the other drag component, skin friction, should be relatively insensitive to grid refinement. Figure 41 shows just that. The OVERFLOW-SA skin friction drag results are nearly unchanged with grid convergence for both constant alpha and constant $C_{L}$ runs. The CFL3D-SST W1 data at constant alpha do show more variation, but the skin friction increases by less than 1 count going from the medium grid to the fine grid.

Pitching moment data is presented in Figure 42. The constant alpha OVERFLOW-SA data indicate W2 has a slight nose-up pitching moment shift relative to W1. The constant $C_{L}$ data actually cross-over at about the medium grid size. Considering the scale used for the pitching moment plots $\left(\Delta C_{M}=.001\right)$, the medium/fine/extra-fine grid data appear to be close to the asymptotic range of grid convergence.

Lift coefficient from the constant angle-of-attack runs is plotted against grid size in Figure 43 . The OVERFLOW-SA results indicate W2 produces about 0.02 more $C_{L}$ at an alpha of $0.5^{\circ}$, and the medium grid W1 data shows CFL3D-SST predicts about 0.008 less $C_{L}$ than OVERFLOW-SA. Once again, the medium/fine/extra-fine grid $C_{L}$ data for both $\mathrm{W} 1$ and $\mathrm{W} 2$ are considered close to asymptotically approaching the continuum.

Grid convergence results for angle-of-attack from the constant $C_{L}$ runs are given in Figure 44 . This plot shows CFL3D and OVERFLOW agree reasonably well on alpha for the W1 coarse and medium grids. The OVERFLOW-SA results show a nearly constant alpha increment for all grid sizes analyzed with W2 requiring roughly $0.2^{\circ}$ less alpha to hold $C_{L}$ constant at 0.5 . As with all other wing-alone OVERFLOW results, the variation in angle-of-attack with grid refinement has a linear character.

\section{IV.C.2. $\quad D P W-W 1 / W 2$ Wing Pressures}

Pressure comparisons from the $\mathrm{W} 1$ wing-alone test case are provided in Figure 45 . The pressures were extracted from the $0.5 C_{L}$ solutions at a mid-wing semispan station of 0.551 . The OVERFLOW-SA data, shown on the left, are consistent with the wing/body $C_{p}$ comparison in that the coarse-grid shock is considerably weaker than the next denser mesh. The CFL3D-SST data, shown on the right, has the same trend for shock strength but it's not nearly as dramatic as the OVERFLOW results. Since this observation is made on both test cases, it can be said with some confidence that the coarse grid does not have adequate resolution to accurately model typical transonic pressure distributions. If the wing shock strength and location is not well predicted, computing absolute drag to an error level of a few percent becomes even more challenging. 


\section{IV.C.3. DPW-W1/W2 Drag Polars, Lift/Pitching Moment Curves, and Streamlines}

The medium grid drag polars are shown in Figure 46. The W1 data (green lines/symbols) compare well between codes for the two conditions run in CFL3D. The W2 OVERFLOW-SA polar (purple line/symbols) has a different shape than the W1 polar with the two crossing-over at $C_{L}$ 's below and above the design point. The W2 drag improvement is roughly 11 counts at the design $C_{L}$ of 0.5 , but this optimized wing performs worse than W1 at the low and high end of the polar. This is not surprising given a single-point optimization was used to generate the W2 wing (see Section II.B).

Lift curves for the medium grid alpha sweep runs are provided in Figure 47. The two CFL3D-SST data points, at $C_{L}$ 's of 0.47 and 0.50 , are very close to the OVERFLOW-SA curve. For $C_{L}$ 's up to about 0.6, the $\mathrm{W} 2$ wing provides the same lift as $\mathrm{W} 1$ with about a $0.2^{\circ}$ reduction in angle-of-attack. At roughly $0.66 C_{L}$, the W2 lift curve crosses-over the W1 curve.

Figure 48 compares the wing-alone pitching moment curves. The CFL3D-SST data show a more noseup moment compared to the OVERFLOW-SA curve at the same $C_{L}$. CFL3D predicts W1 gives roughly 0.002 more nose-up moment than OVERFLOW at the design point. The W1/W2 OVERFLOW comparison indicates a difference in how the flow separates across the span with angle-of-attack.

Figures 49, 50, and 51 compare W1/W2 upper-surface streamlines at alphas of $0.5^{\circ}, 1.5^{\circ}$, and $2.5^{\circ}$, respectively. These streamline images were generated using medium grid OVERFLOW-SA solutions. The streamlines at $0.5^{\circ}$ alpha show attached flow for both wings. It's actually more interesting to note the difference in upper-surface pressure contours in Figure 49. At this angle-of-attack, the W2 shock is clearly weaker. The next comparison is made at $1.5^{\circ}$ because this is the approximate angle-of-attack where flow separation begins at the foot of the shock. Figure 51 shows that, at $2.5^{\circ}$, the upper-surface flow is completely separated downstream of the shock on both wings.

\section{Conclusions}

The DLR-F6 wing/body with and without the FX2B fairing and the DPW-W1/W2 wing-alone configurations from the third Drag Prediction Workshop were analyzed using the two widely-used CFD codes CFL3D and OVERFLOW. A parametrically equivalent set of overset grids were generated by first building a grid based on guidelines for cruise drag prediction studies. This grid, referred to as the medium-sized grid, was modified by reducing and increasing the total number of grid points by a factor of 3.5 to create the coarse and fine grids. The fine grid was then enhanced again by the same factor to produce the extra-fine grid. The CFD results from these uniformly refined grid families were post-processed using Richardson extrapolation which, in theory, can be used to establish grid resolution requirements for accurate drag prediction. This grid convergence study showed that the low resolution coarse grid data is insufficient for accurate drag prediction. This is due, in part, to differences in computed shock strength and location, where the coarse grid solution stands apart from the rest for both flow solvers and configurations analyzed.

With the use of a fourth extra-fine mesh, it appears that the medium/fine/extra-fine results on the FX2B configuration fall within the asymptotic range of grid convergence. However, this is not the case with the baseline DLR-F6 geometry. Furthermore, the medium/fine/extra-fine results of both DPW-W1 and DPW-W2 wings appear to be within the asymptotic range. Based on these findings, it seems that moderately-sized pockets of flow separation can be a root cause for poor grid convergence. This conslusion is somewhat in contrast to that of Reference 23, which assessed results from a much larger community with wider variation in grid types, grid quality, and turbulence models. The conclusion herein is based on OVERFLOW results on overset meshes.

To illustrate the impact of the F6 side-of-body separation on grid convergence, the computed drag from the fine and extra-fine grids were extrapolated to the continuum to approximate grid-converged drag. Using the results of this extrapolation, the FX2B drag increment (FX2B-F6) from an infinitely-refined grid was 8 counts larger than the increment computed using the medium grid results. Making the same type of comparison for the wing-alone case, the drag increment (W2-W1) from an infinitely-refined grid was 0.7 counts lower than the medium grid increment. This exercise shows that the error in incremental drag associated with a medium-sized grid analysis drops by an order of magnitude when separated flow is not present.

The wing/body and wing-alone medium grids were analyzed at multiple angles-of-attack to establish drag polars and lift/pitching moment curves. Two turbulence models, the one-equation Spalart-Allmaras and the two-equation Menter SST, were used for some of the DLR-F6 OVERFLOW runs. They yielded similar results with less than 5 counts difference in fixed- $C_{L}$ drag coefficient and a nearly identical drag polar shape. 
Comparing the code-to-code results qualitatively, CFL3D exhibited a wavy character in its drag polar, whereas OVERFLOW was smooth. Other than this discrepancy, the alpha-sweep results from the two codes compared well. The following list summarizes areas where CFL3D and OVERFLOW produced similar wing/body results.

- Trends in incremental data (e.g., FX2B had considerably less drag than F6 through the mid- $C_{L}$ range)

- The size of the DLR-F6 side-of-body separation bubble and its variation with grid refinement and viscous effects

- No DLR-F6 wing trailing-edge separation at 5 million Reynolds number

- DLR-F6 wing pressures - namely shock strengh and location on medium and fine grids

The last three items in this list are based on runs made at the design point $C_{L}$ of 0.5 . The wing-alone results show the two codes compute nearly the same drag for W1 at $C_{L}$ 's near the design point, but more CFL3D data is needed to comment on incremental trend comparisons for this test case.

\section{Appendix}

During the process of writing this paper, a discrepancy was found in the CFL3D FX2B results originally submitted to the workshop: the medium grid data computed for the grid convergence study was noted to be different than the alpha sweep data. The alpha sweep runs were made as a series of restarts using the lower alpha solutions as a starting point for the next higher alpha. Since the grid convergence study required only one data point, those runs were all made from "scratch" (freestream levels). As a trouble-shooting exercise, a few of the alpha sweep runs were subsequently made from scratch and slightly different answers (as much as 3 drag counts) were obtained. The authors are unable to explain this hysteresis-type of dependence on initial conditions. All solutions were considered fully converged with residuals dropping several orders of magnitude. Furthermore, the force and moment convergence histories "leveled out" (did not oscillate). This hysteresis behavior did not occur for the F6 case.

Note that although the grids are the same, CFL3D and OVERFLOW did not use the same overset interpolants. Because CFL3D is a cell-centered solver, it could not use the interpolants provided for the node-based OVERFLOW solver; they had to be regenerated. The current method for doing this is an ad-hoc process. ${ }^{17}$ For the FX2B medium grid, this process yielded nearly five times as many orphans than for the F6 grid (which contained 250 orphan points). There were only five orphans in the OVERFLOW grids for both F6 and FX2B. It is possible that issues related to the interpolants/orphans contributed to the discrepancy in FX2B results.

\section{Acknowledgment}

The authors acknowledge The Boeing Company and The National Aeronautics and Space Administration for their support in the Drag Prediction Workshop series.

\section{References}

${ }^{1}$ M. J. Hemsch. Statistical analysis of CFD solutions from the Drag Prediction Workshop. AIAA paper 2002-0842, Reno, NV, January 2002.

${ }^{2}$ M. Hemsch. Statistical analysis of CFD solutions from the drag prediction workshops. In CFD-based Aircraft Drag Prediction and Reduction, Hampton, VA, November 2003. von Karman Institue Lecture Series.

${ }^{3}$ D. W. Levy, J. C. Vassberg, R. A. Wahls, T. Zickuhr, S. Agrawal, S. Pirzadeh, and M. J. Hemsch. Summary of data from the first AIAA CFD Drag Prediction Workshop. AIAA paper 2002-0841, Reno, NV, January 2002.

${ }^{4} 1^{\text {st }}$ AIAA CFD Drag Prediction Workshop. http://aaac.larc.nasa.gov/tsab/cfdlarc/aiaa-dpw/Workshop1/workshop1.html, June 2001.

${ }^{5}$ K. R. Laflin, J. C. Vassberg, R. A. Wahls, J. H. Morrison, O. Brodersen, M. Radowitz, E. N. Tinoco, and J. Godard. Summary of data from the second AIAA CFD drag prediction workshop. AIAA Paper 2004-0555, $42^{\text {nd }}$ AIAA Aerospace Sciences Meeting and Exhibit, Reno, NV, January 2004.

${ }^{6} 2^{\text {nd }}$ AIAA CFD Drag Prediction Workshop. http://aaac.larc.nasa.gov/tsab/cfdlarc/aiaa-dpw/, dpw@cessna.textron.com, June 2003.

${ }^{7}$ J. C. Vassberg, A. J. Sclafani, and M. A. DeHaan. A wing-body fairing design for the DLR-F6 model: a DPW-III case study. AIAA Paper 2005-4730, AIAA 23 ${ }^{\text {rd }}$ Applied Aerodynamics Conference, Toronto, Canada, June 2005.

${ }^{8}$ A. J. Sclafani, M. A. DeHaan, and J. C. Vassberg. OVERFLOW drag predictions for the DLR-F6 transport configuration: A DPW-II case study. AIAA Paper 2004-0393, 42 ${ }^{\text {nd }}$ AIAA Aerospace Sciences Meeting and Exhibit, Reno, NV, January 2004. 
${ }^{9}$ O. Brodersen and A. Sturmer. Drag prediction of engine-airframe interference effects using unstructured Navier-Stokes calculations. AIAA Paper 2001-2414, 19 ${ }^{\text {th }}$ AIAA Applied Aerodynamics Conference, Anaheim, CA, June 2001.

${ }^{10} 3^{\text {rd }}$ AIAA CFD Drag Prediction Workshop. http://aaac.larc.nasa.gov/tsab/cfdlarc/aiaa-dpw/, dpw@cessna.textron.com, June 2006.

${ }^{11}$ J. C. Vassberg, M. A. DeHaan, and A. J. Sclafani. Grid generation requirements for accurate drag predictions based on OVERFLOW calculations. AIAA Paper 2003-4124, $16^{\text {th }}$ AIAA Computational Fluid Dynamics Conference, Orlando, FL, June 2003.

${ }^{12}$ W. M. Chan, R. J. Gomez-III, S. E. Rogers, and P. G. Buning. Best practices in overset grid generation. AIAA Paper 20023191, 32nd AIAA Fluids Dynamics Conference, St. Louis, MO, June 2002.

${ }^{13}$ W. M. Chan, S. E. Rogers, S. M. Nash, P. G. Buning, and R. L. Meakin. User's manual for Chimera Grid Tools, version 1.9. http://people.nas.nasa.gov/ wchan/cgt/doc/man.html, NASA Ames Research Center, September 2004.

${ }^{14}$ W. M. Chan. The OVERGRID interface for computational simulations on overset grids. AIAA Paper 2002-3188, 32 ${ }^{\text {nd }}$ AIAA Fluids Dynamics Conference, St. Louis, MO, June 2002.

${ }^{15}$ N. E. Shus, W. E. Dietz, S. M. Nash, M. D. Baker, and S. E. Rogers. PEGASUS user's manual version 5.1c. Technical report, MICRO CRAFT, July 2000. 5.0 .

${ }^{16}$ S. L. Krist, R. T. Biedron, and C. L. Rumsey. CFL3D User's Manual. NASA-TM 1998-208444, NASA, June 1998. Version

${ }^{17}$ CFL3D Version 6.4. http://cfl3d.larc.nasa.gov/Cfl3dv6/cfl3dv6.html, 2006.

${ }^{18}$ F. R. Menter. Two-equation eddy-viscosity turbulence models for engineering applications. AIAA Journal, Vol. 32, No. 8, 1994.

${ }^{19}$ P. G. Buning, D. C. Jespersen, T. H. Pulliam, W. M. Chan, J. P. Slotnick, S. E. Krist, and K. J. Renze. OVERFLOW user's manual, version 1.81. NASA Report, NASA Langley Research Center, Hampton, VA, 1999.

${ }^{20}$ P. R. Spalart and S. R. Allmaras. A one-equation turbulence model for aerodynamic flows. La Recherche Aerospatiale, No. 1, 1994.

${ }^{21}$ M. D. Salas. Digital flight: The last cfd aeronautical grand challenge. Journal of Scientific Computing, Vol. 28, No. 213, September 2006.

${ }^{22}$ D. J. Mavriplis. Grid resolution study of a drag prediction workshop configuration using the NSU3D unstructured mesh solver. AIAA Paper 2005-4729, AIAA 23 rd Applied Aerodynamics Conference, Toronto, Canada, June 2005.

${ }^{23}$ J. C. Vassberg, E. N. Tinoco, M. Mani, O. P. Brodersen, B. Eisfeld, R. A. Wahls, J. H. Morrison, T. Zickuhr, K. R. Laflin, and D. J. Mavriplis. Summary of the third AIAA cfd drag prediction workshop. AIAA Paper 2007-0260, 45 ${ }^{\text {th }}$ AIAA Aerospace Sciences Meeting and Exhibit, Reno, NV, January 2007. 


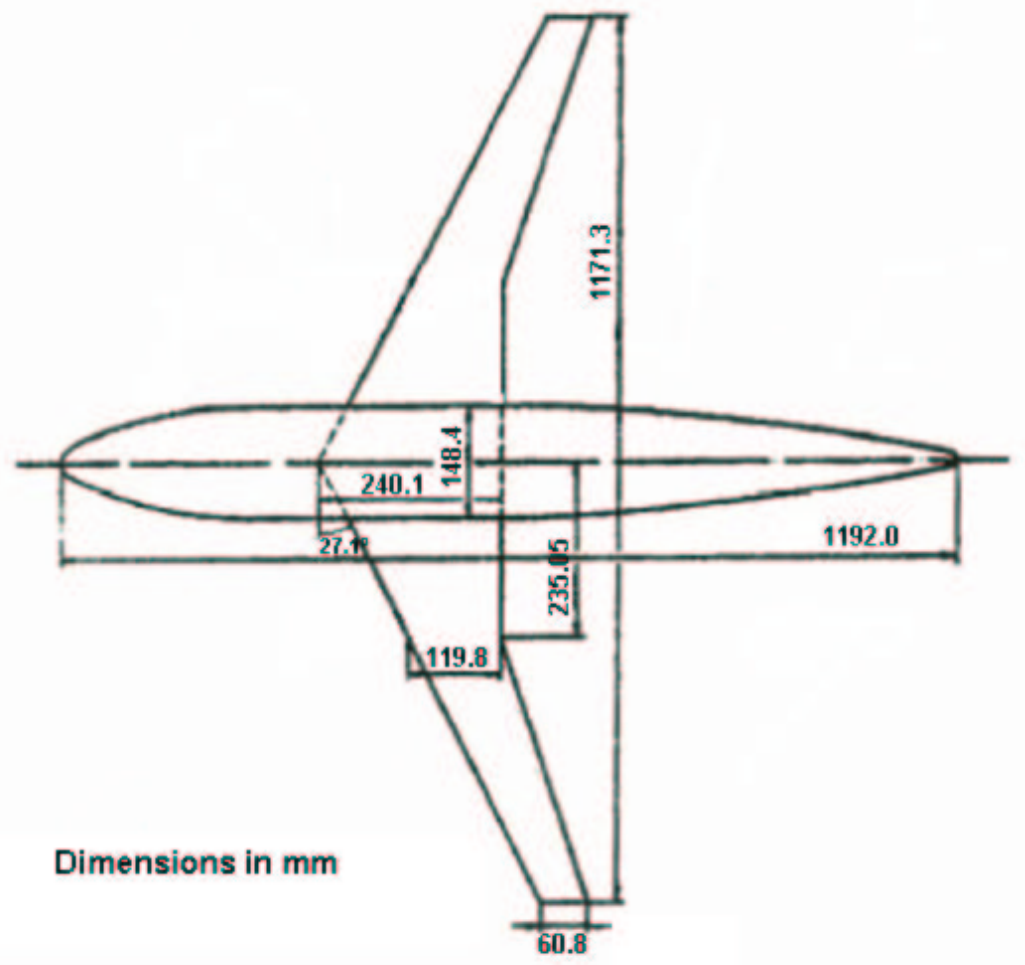

Figure 1. DLR-F6 Wing/Body Planform.

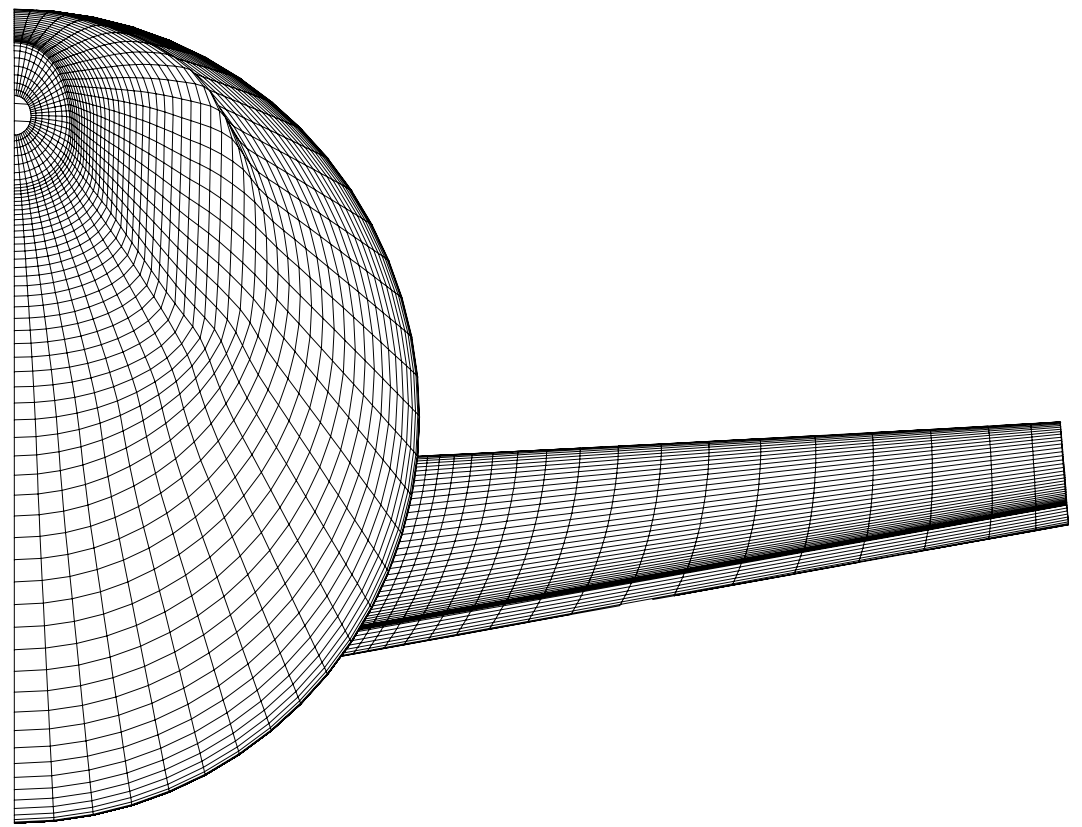

Figure 2. Rear View of Baseline DLR-F6 Fuselage and Wing Root. 


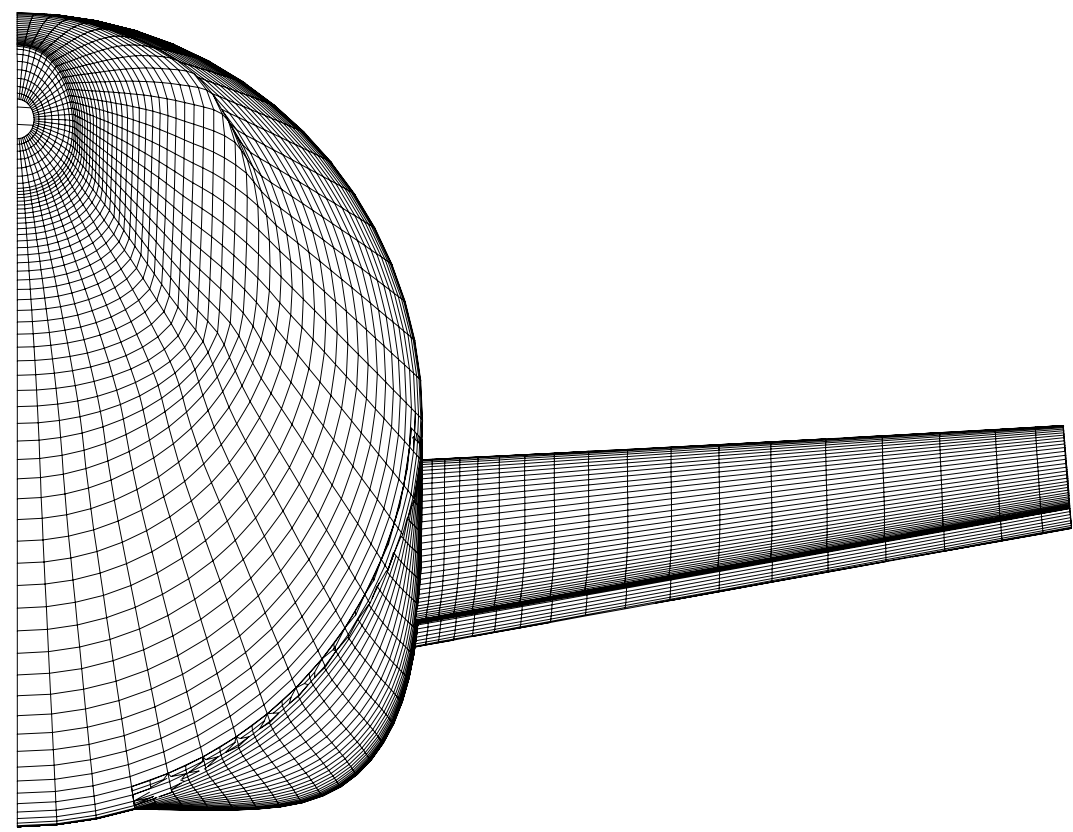

Figure 3. Rear View of Baseline DLR-F6 Fuselage with FX1 WB Fairing.

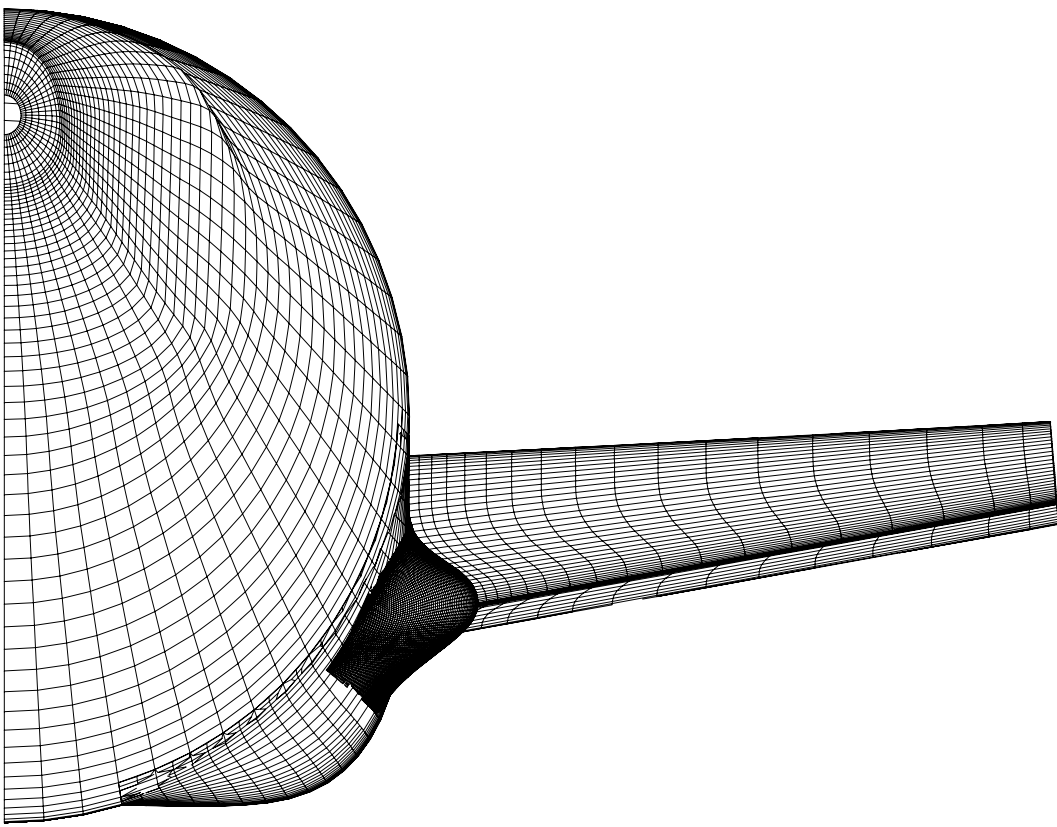

Figure 4. Rear View of Baseline DLR-F6 Fuselage with FX2 WB Fairing. 


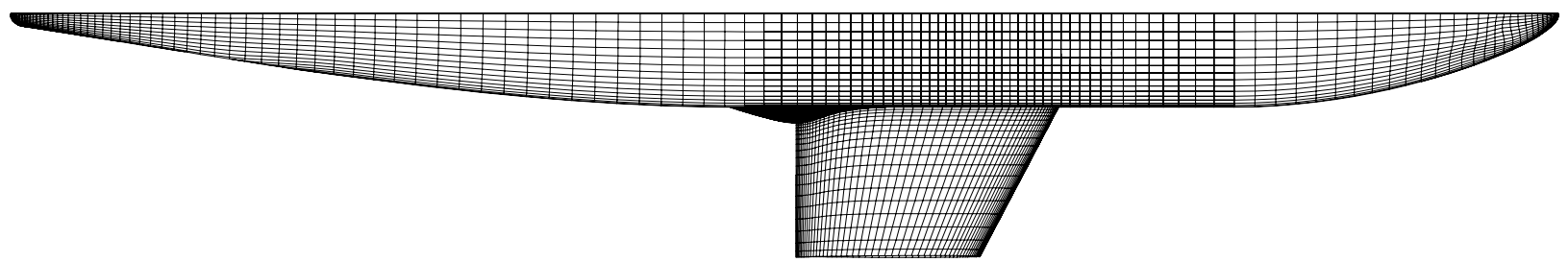

Figure 5. Top View of Baseline DLR-F6 Fuselage with FX2 WB Fairing.

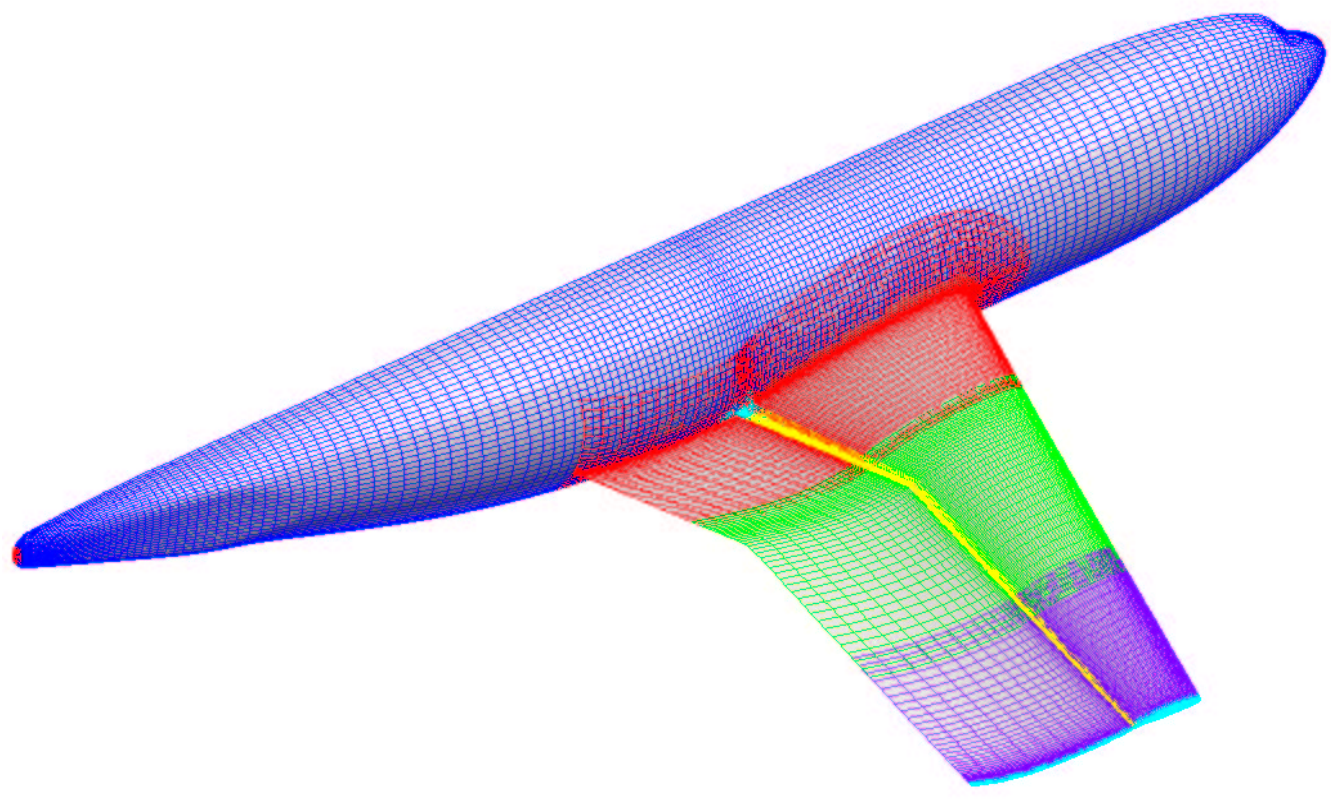

Figure 6. DLR-F6 WB Overset Surface Grids. 


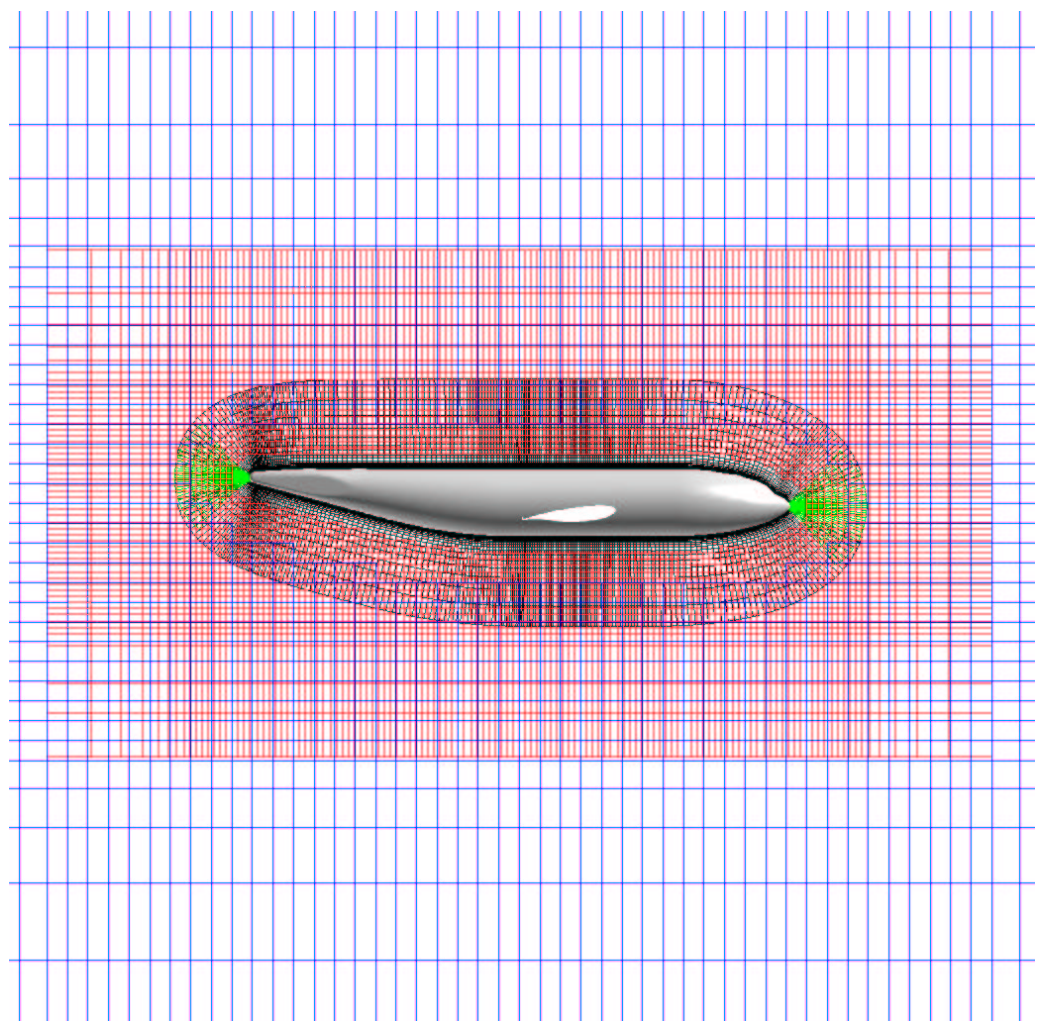

Figure 7. DLR-F6 WB Volume Grid at Symmetry Plane.

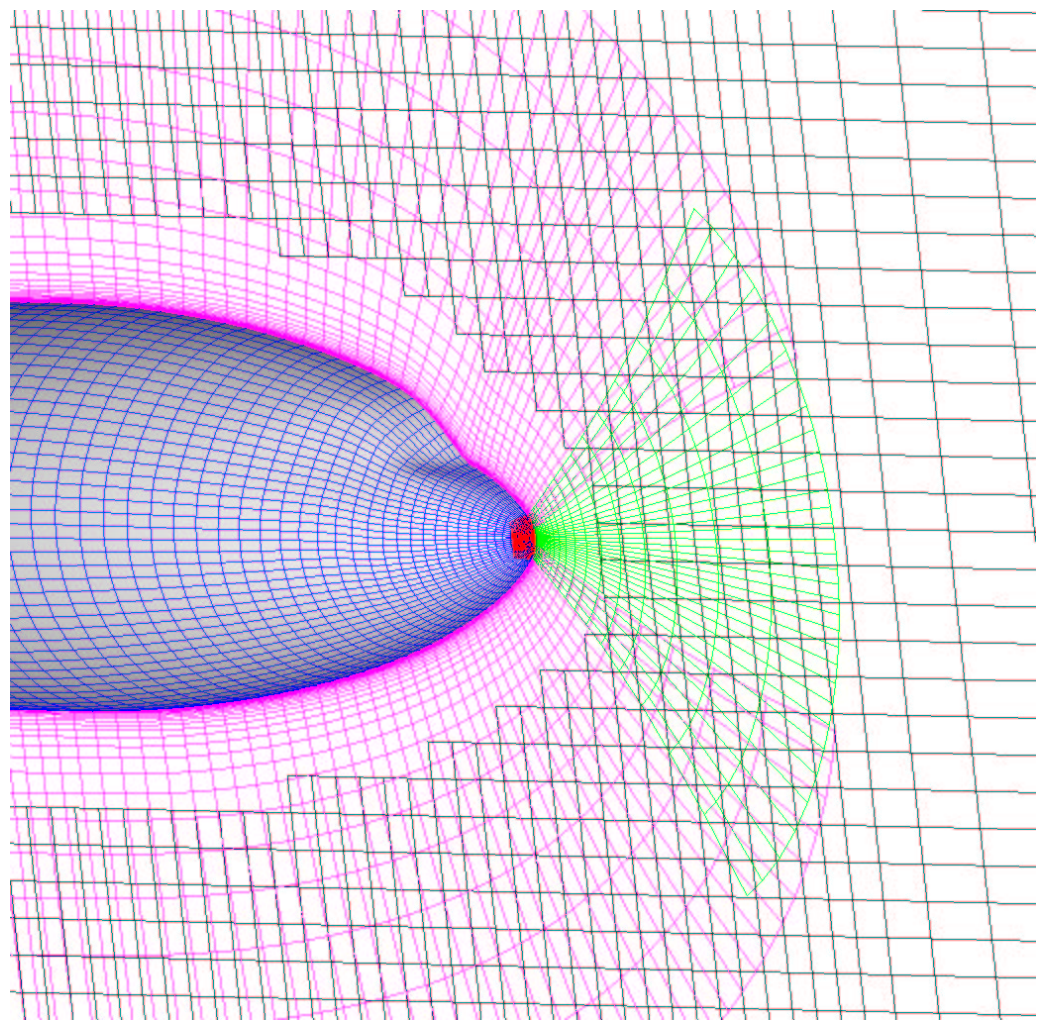

Figure 8. DLR-F6 WB Volume Grid at Symmetry Plane - Nose Region. 


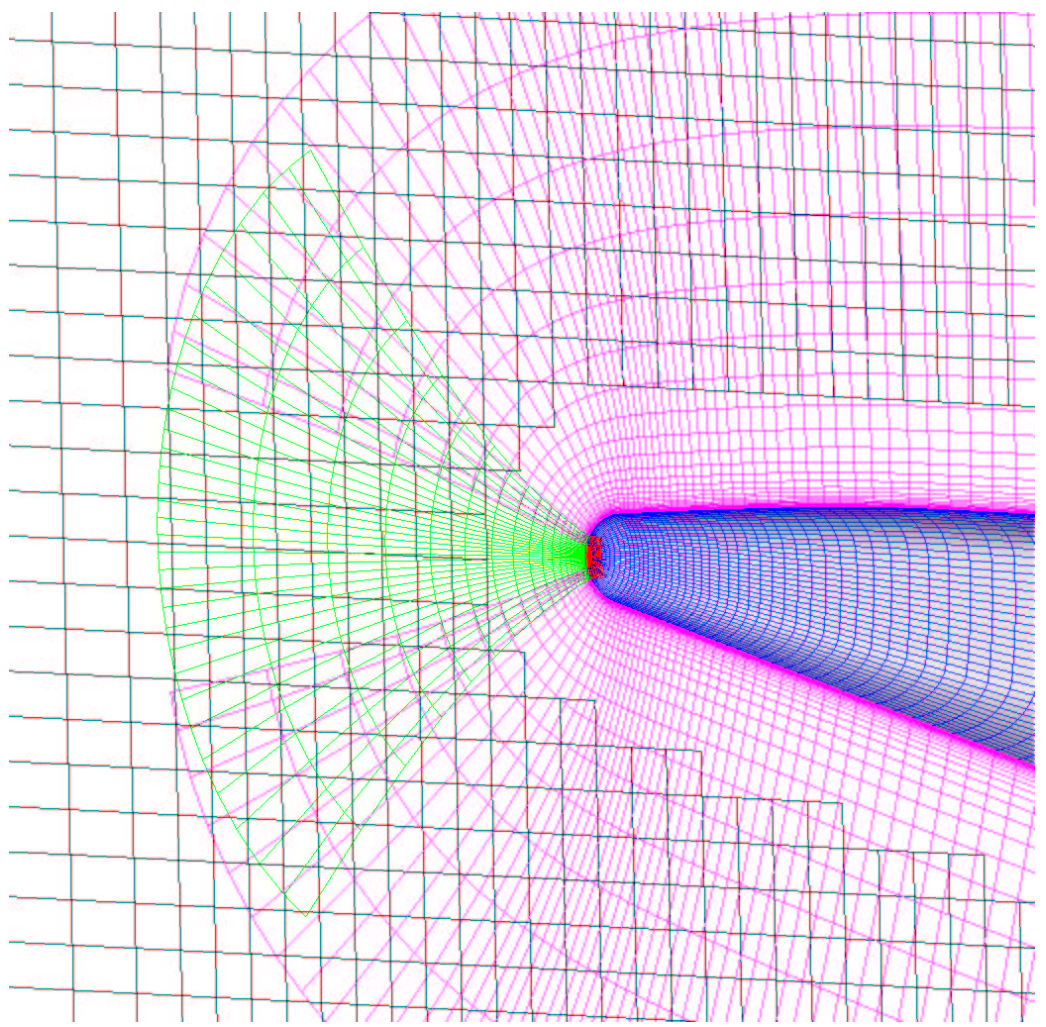

Figure 9. DLR-F6 WB Volume Grid at Symmetry Plane - Tail Region.

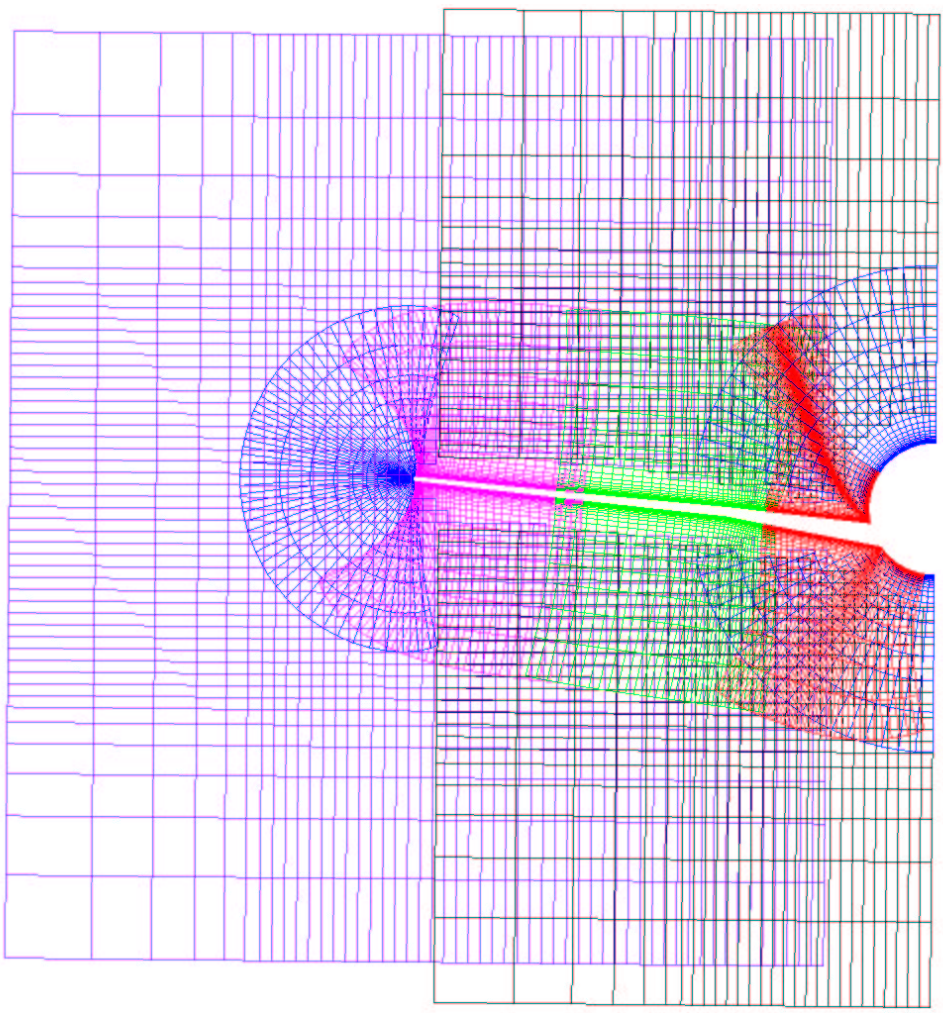

Figure 10. DLR-F6 WB Volume Grid at Wing Mid-Chord. 


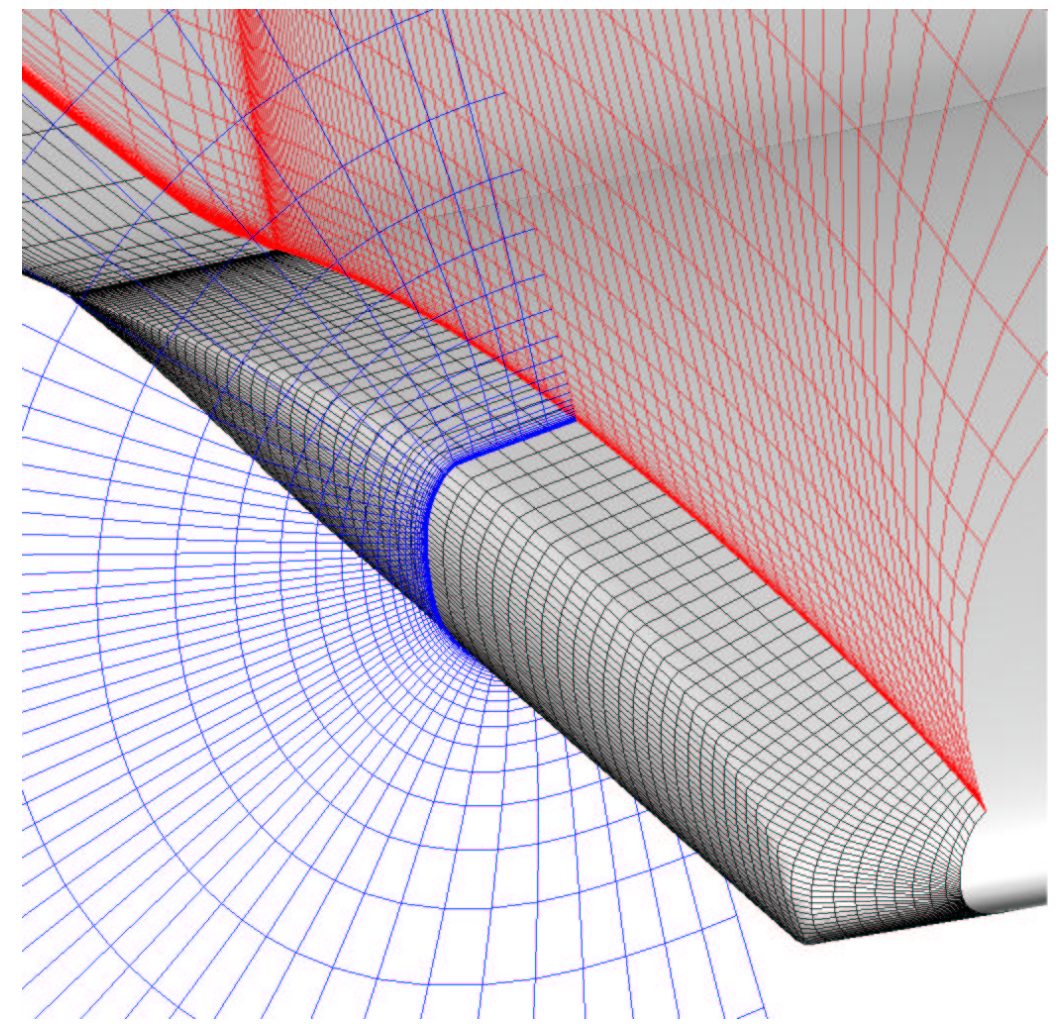

Figure 11. DLR-F6 WB Volume Grid at Wing Tip.

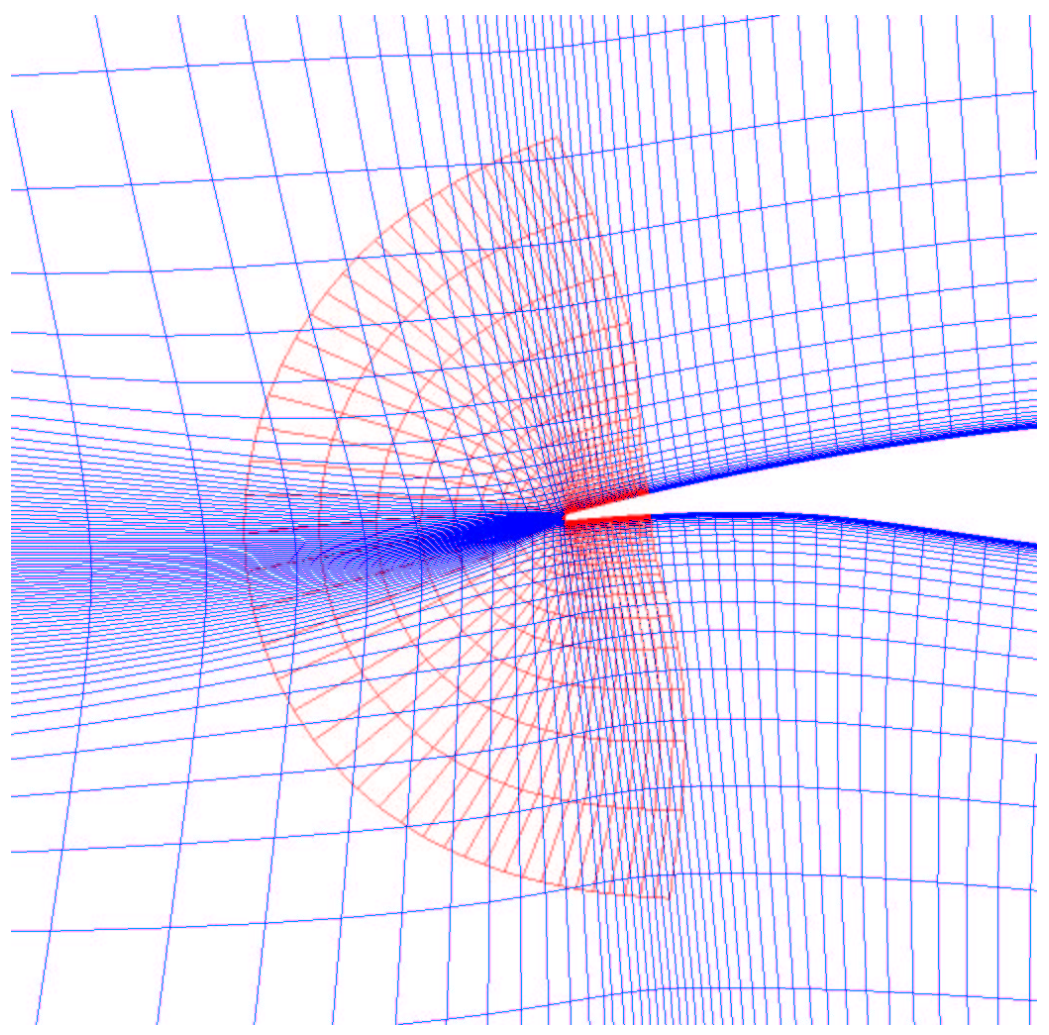

Figure 12. DLR-F6 WB Volume Grid at Wing Trailing-Edge. 


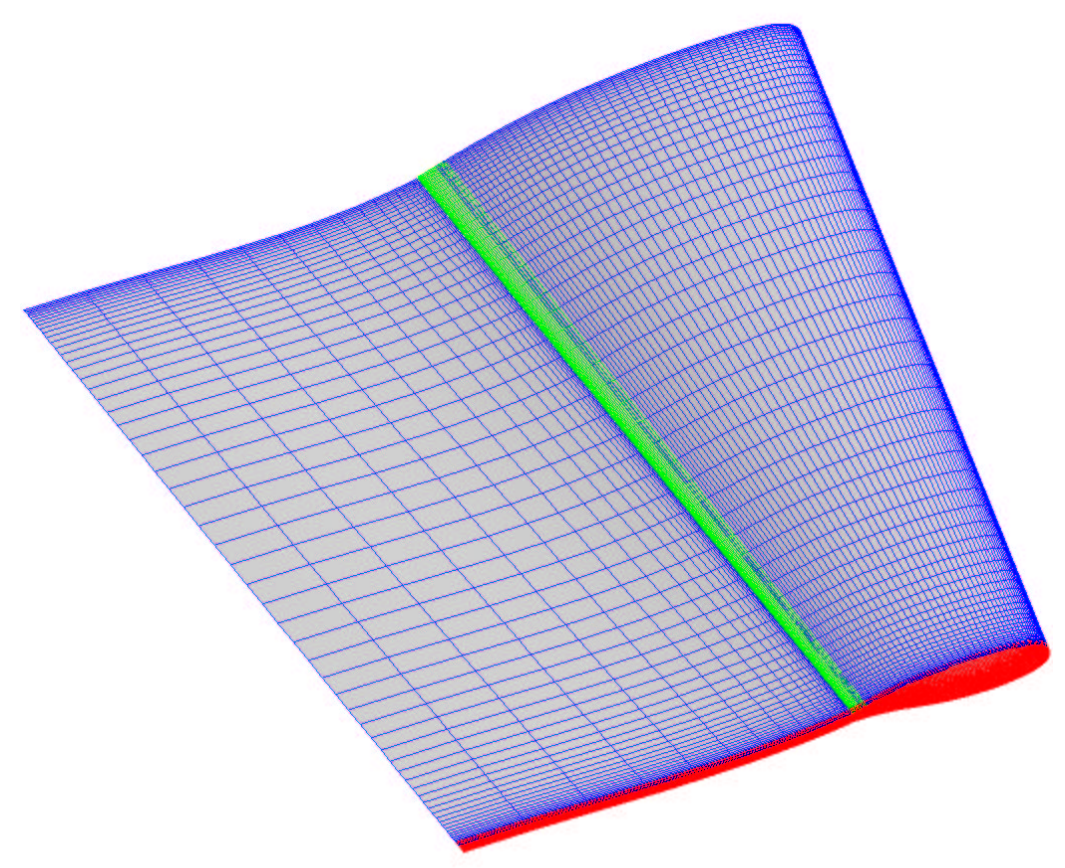

Figure 13. DPW-W1/W2 Surface Grid Layout.

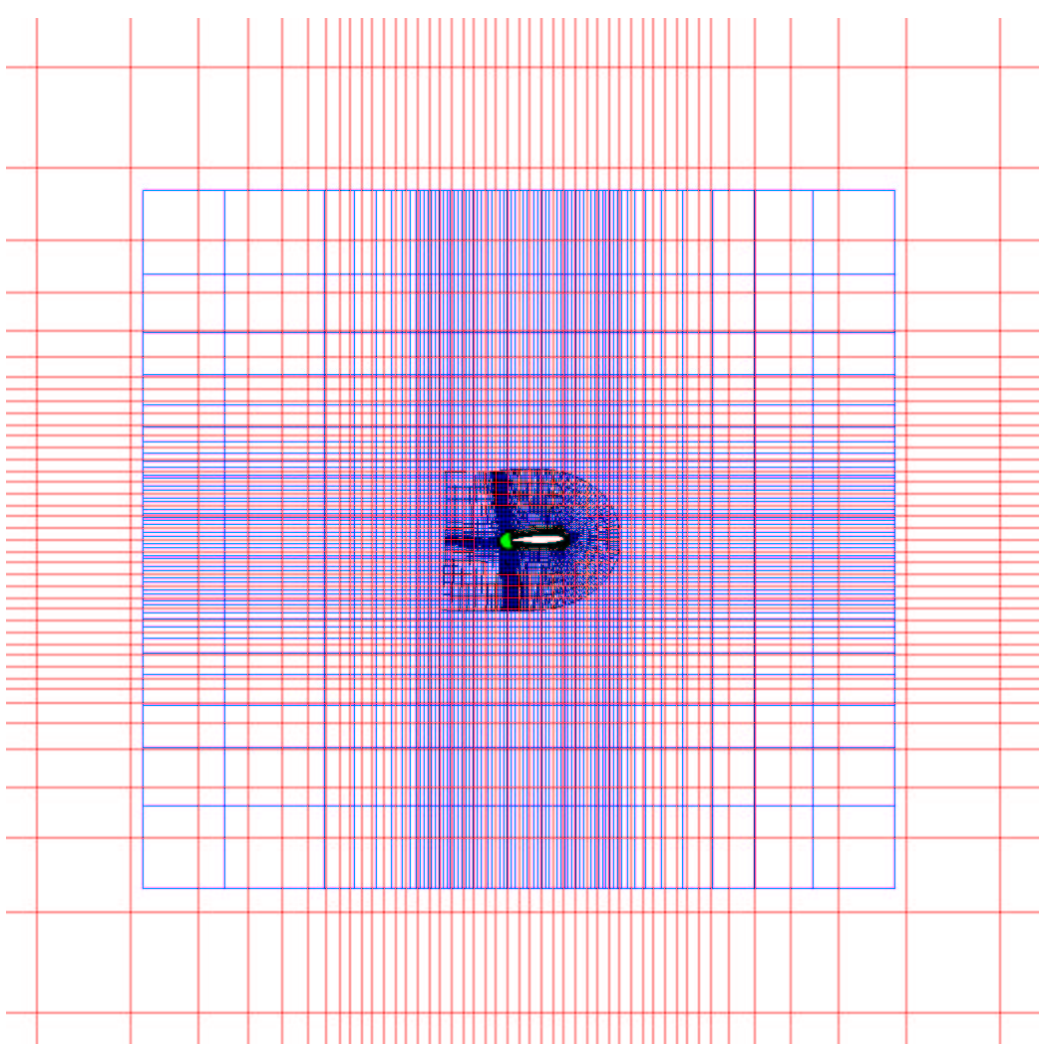

Figure 14. DPW-W1/W2 Volume Grid at Symmetry Plane. 


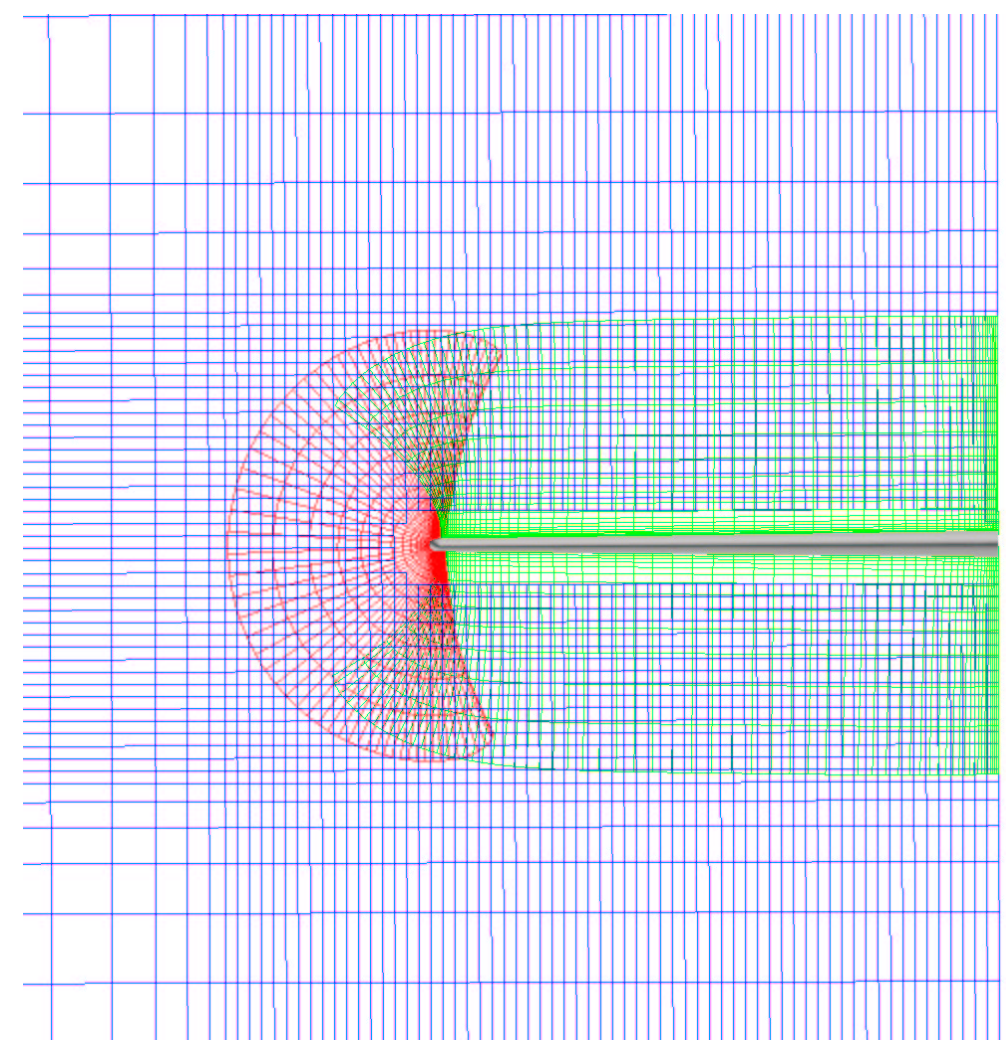

Figure 15. DPW-W1/W2 Volume Grid at Wing Mid-Chord.

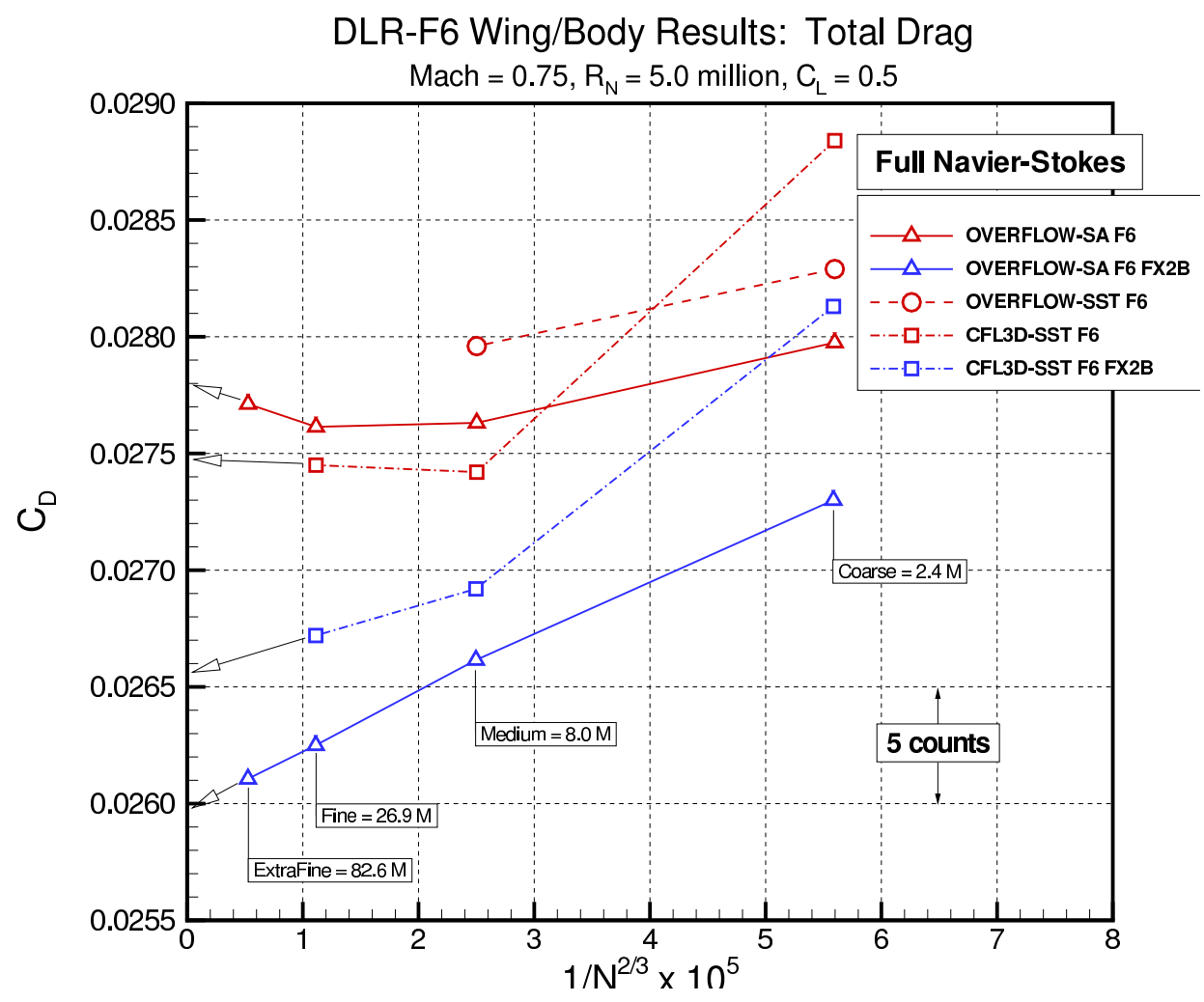

Figure 16. DLR-F6 Grid Convergence Study: Full Navier-Stokes Total Drag. 


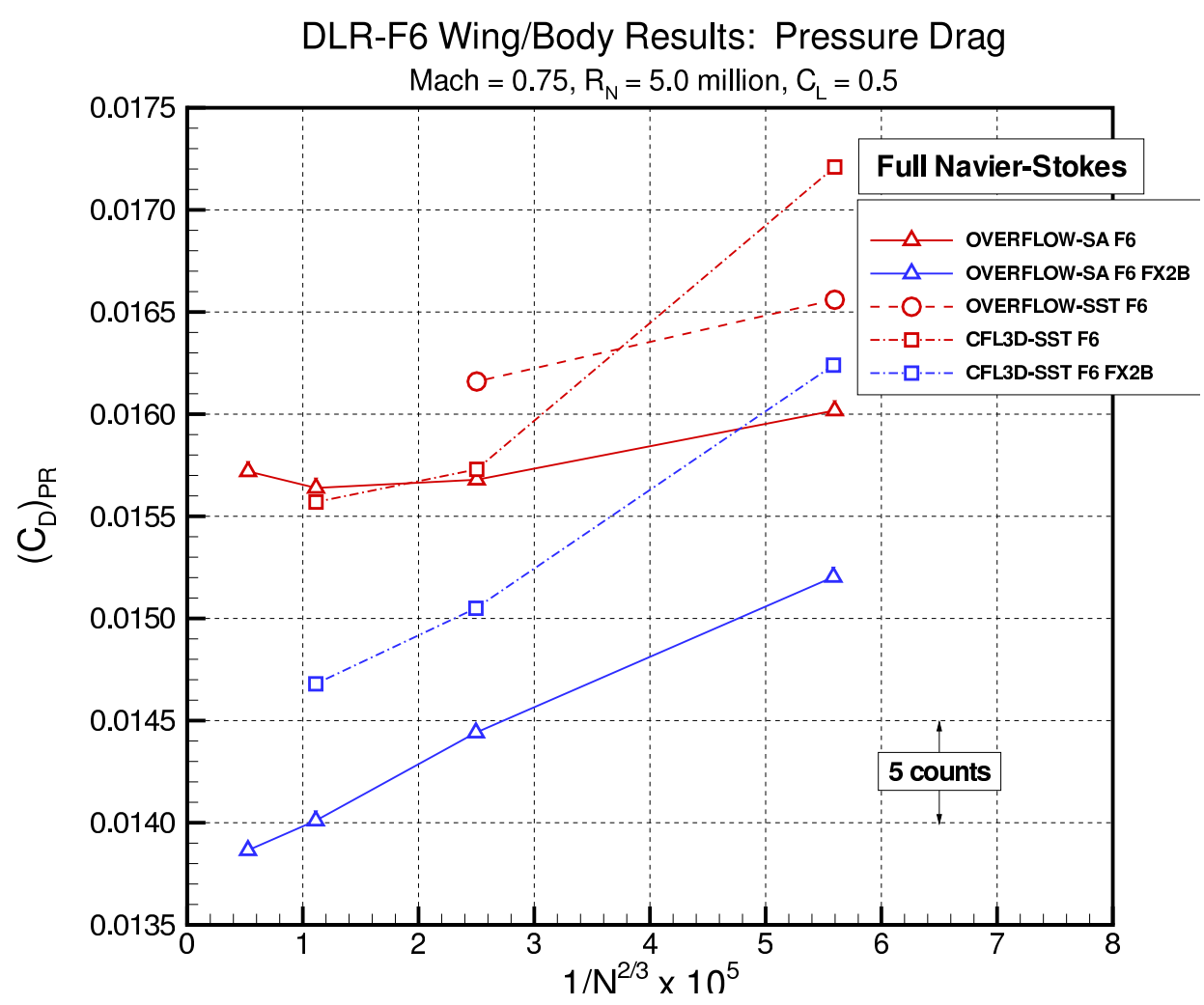

Figure 17. DLR-F6 Grid Convergence Study: Full Navier-Stokes Pressure Drag.

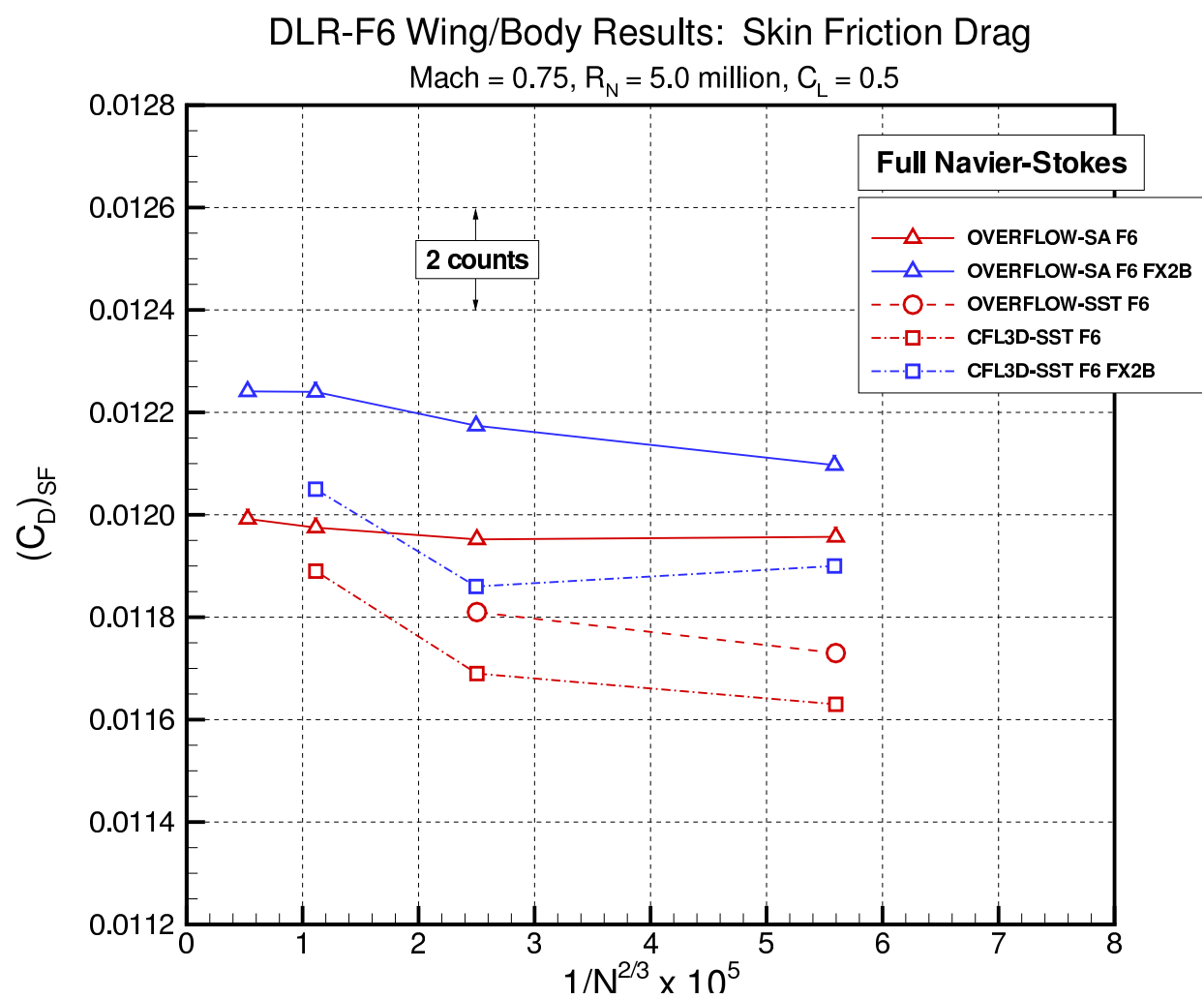

Figure 18. DLR-F6 Grid Convergence Study: Full Navier-Stokes Skin Friction Drag. 


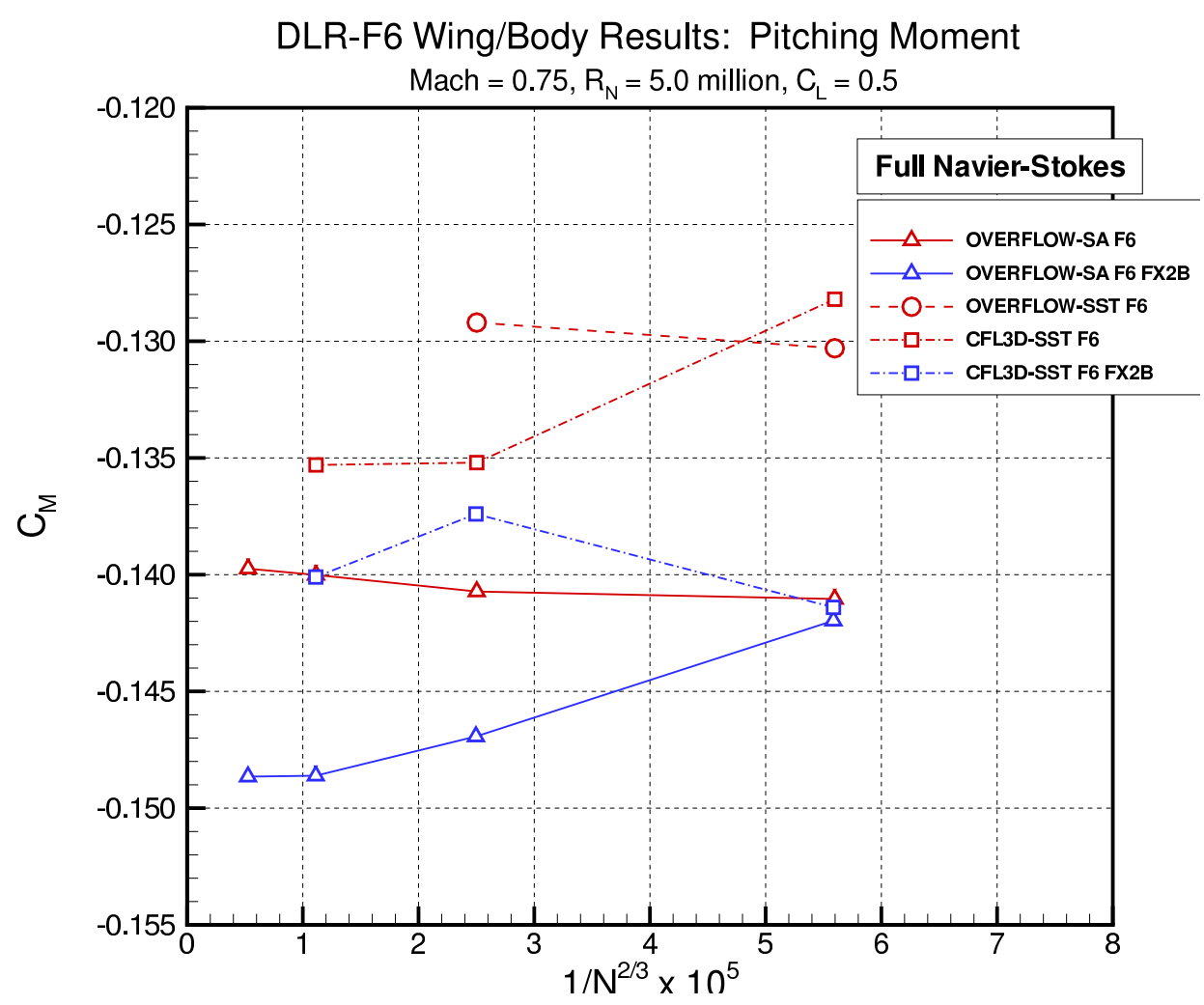

Figure 19. DLR-F6 Grid Convergence Study: Full Navier-Stokes Pitching Moment.

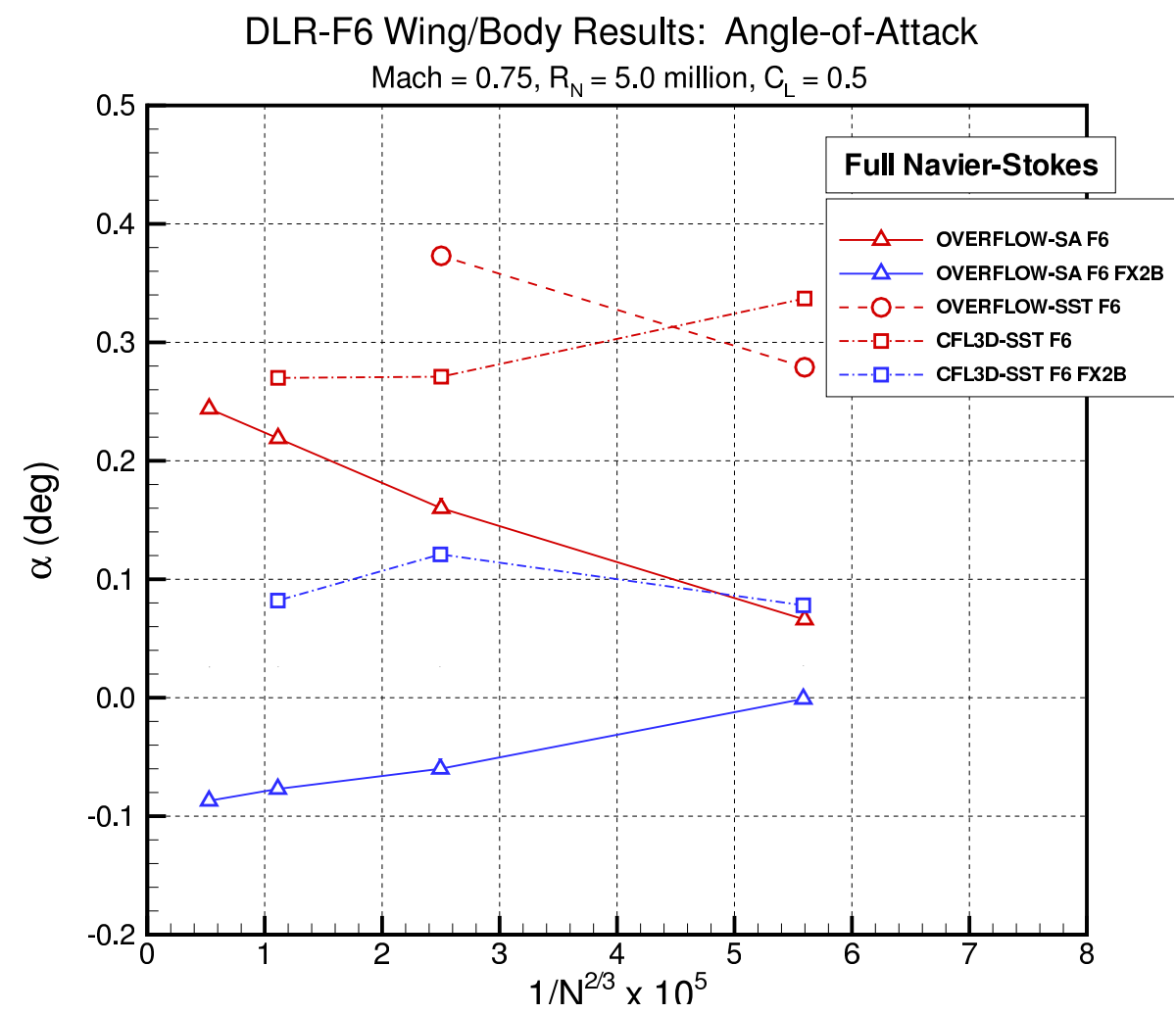

Figure 20. DLR-F6 Grid Convergence Study: Full Navier-Stokes Angle-of-Attack. 


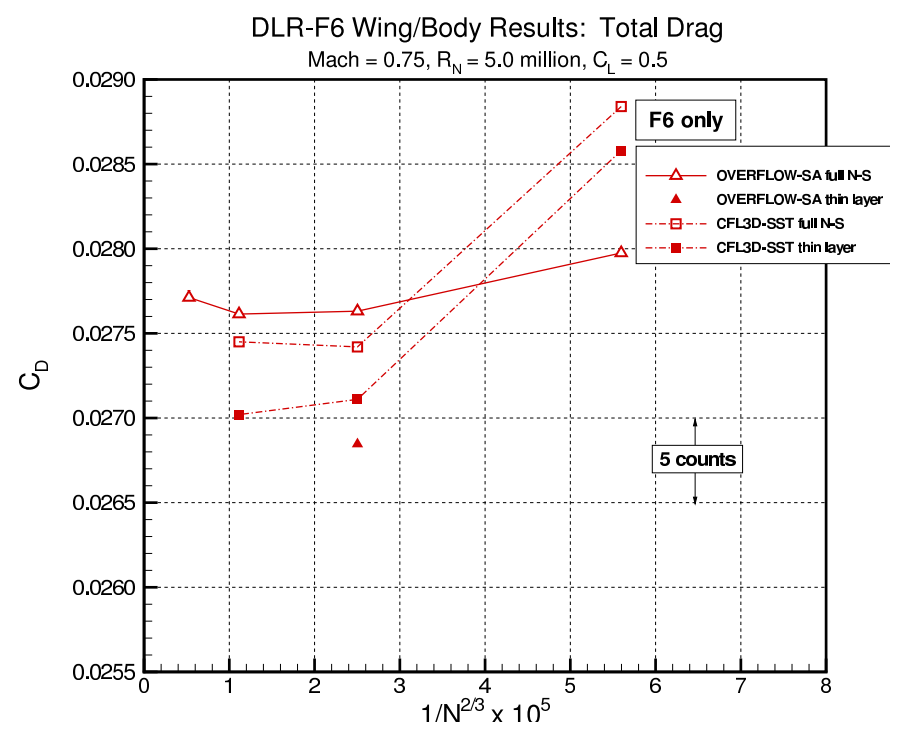

(a) F6 Results.

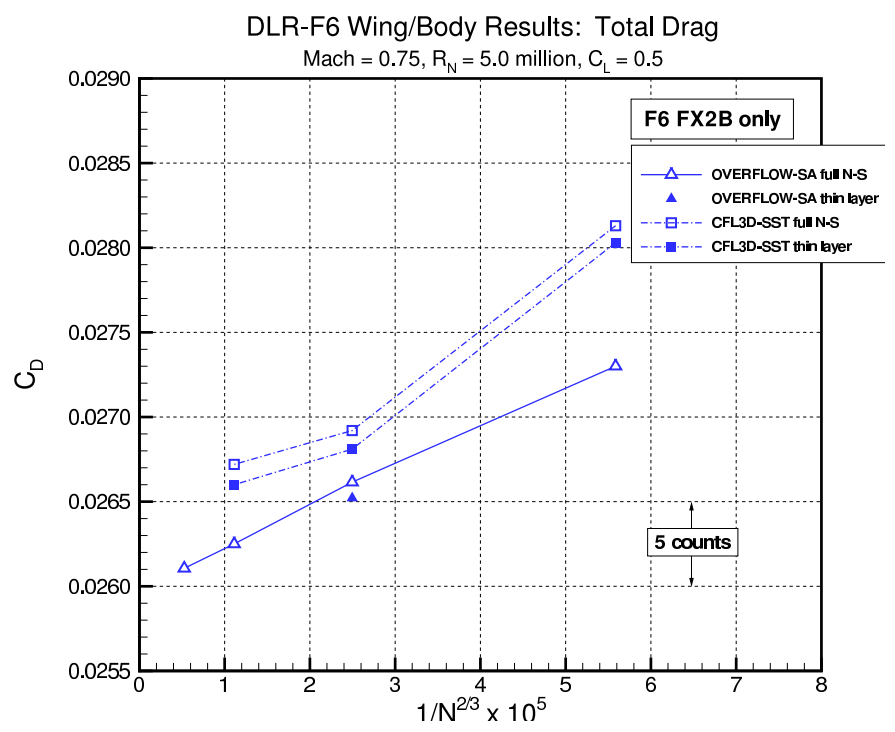

(b) FX2B Results.

Figure 21. DLR-F6 Grid Convergence Study: Thin-Layer Effect.
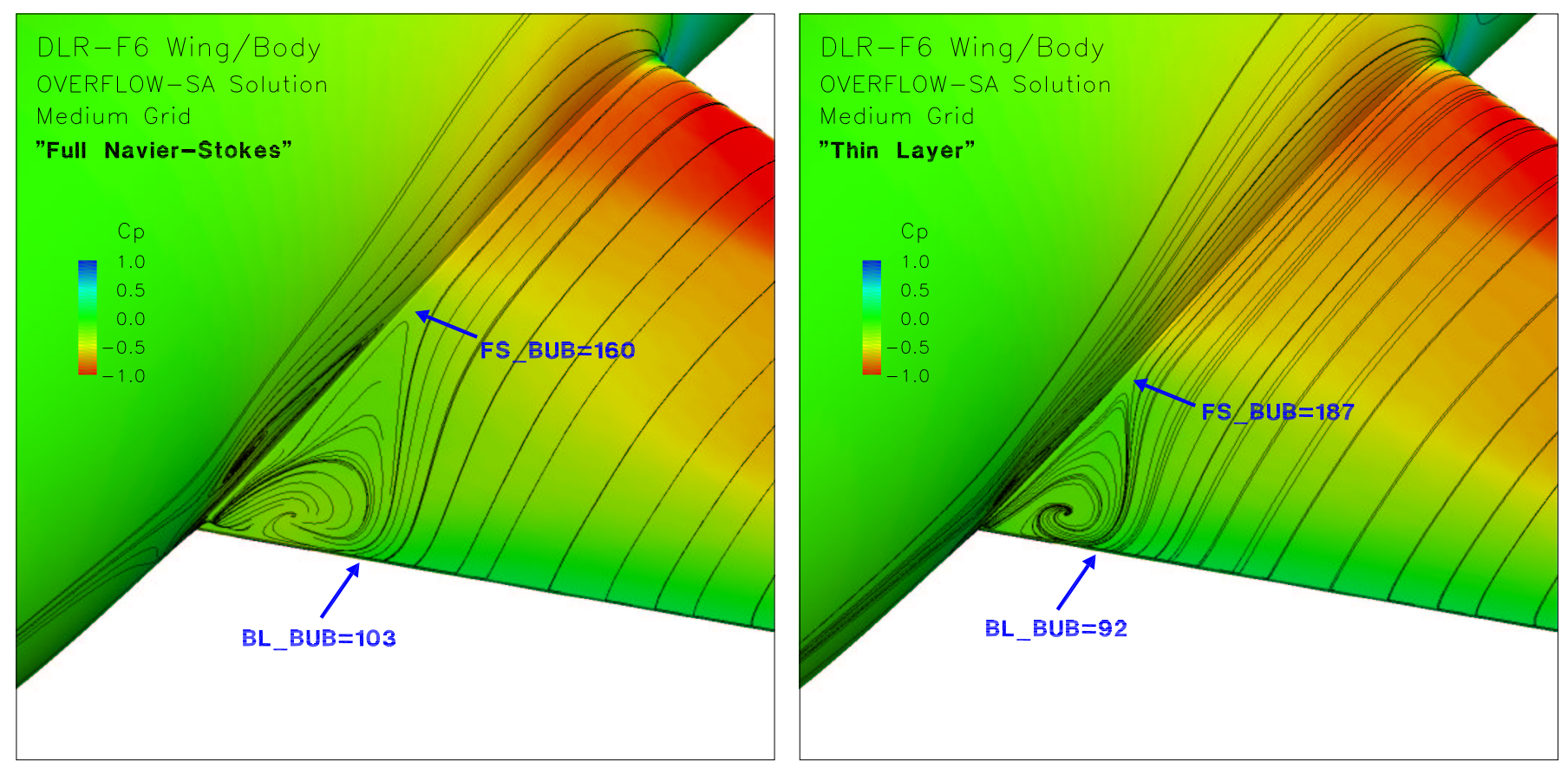

Figure 22. DLR-F6 OVERFLOW-SA Surface Streamline Comparison. 


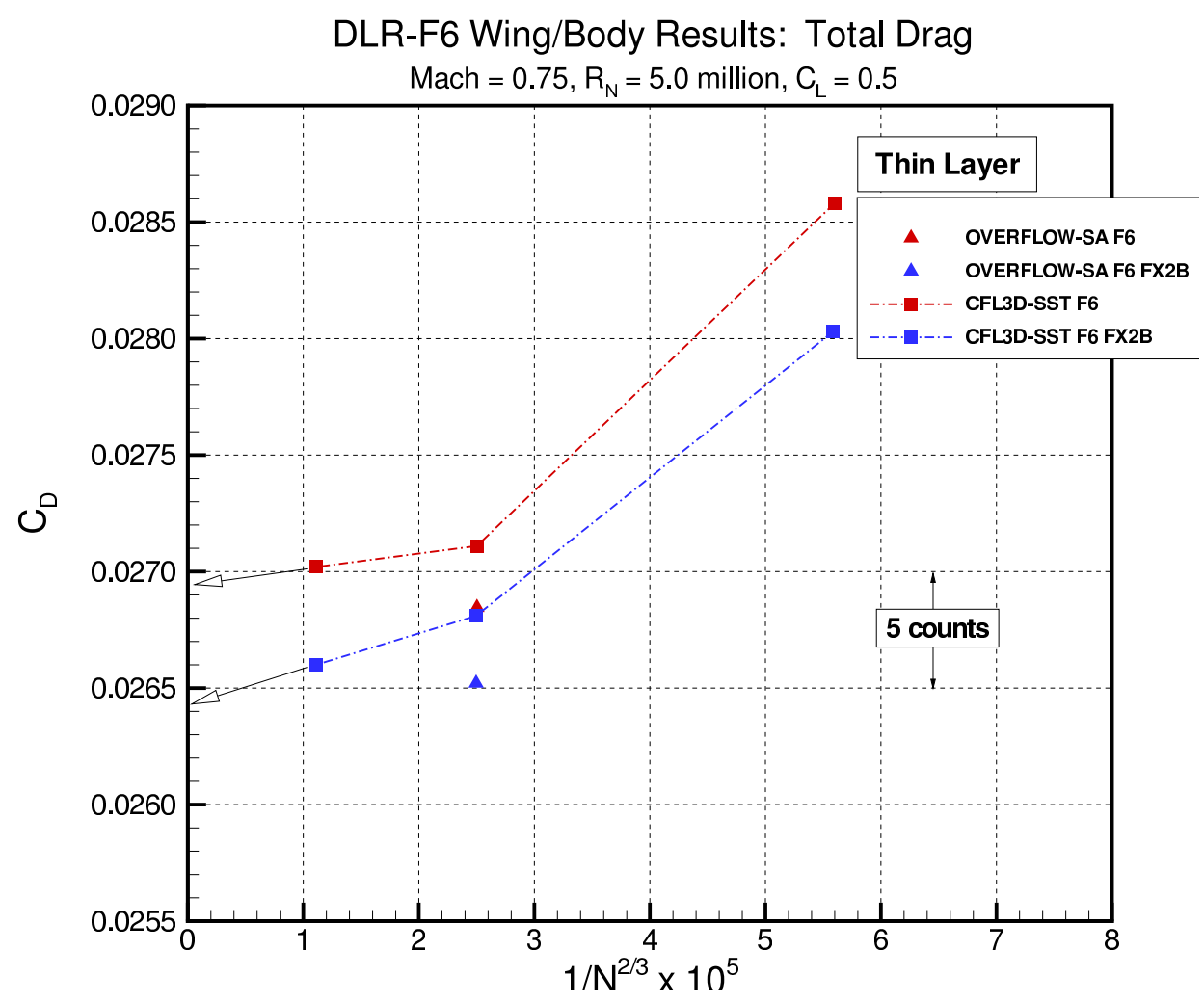

Figure 23. DLR-F6 Grid Convergence Study: Thin-Layer Total Drag.
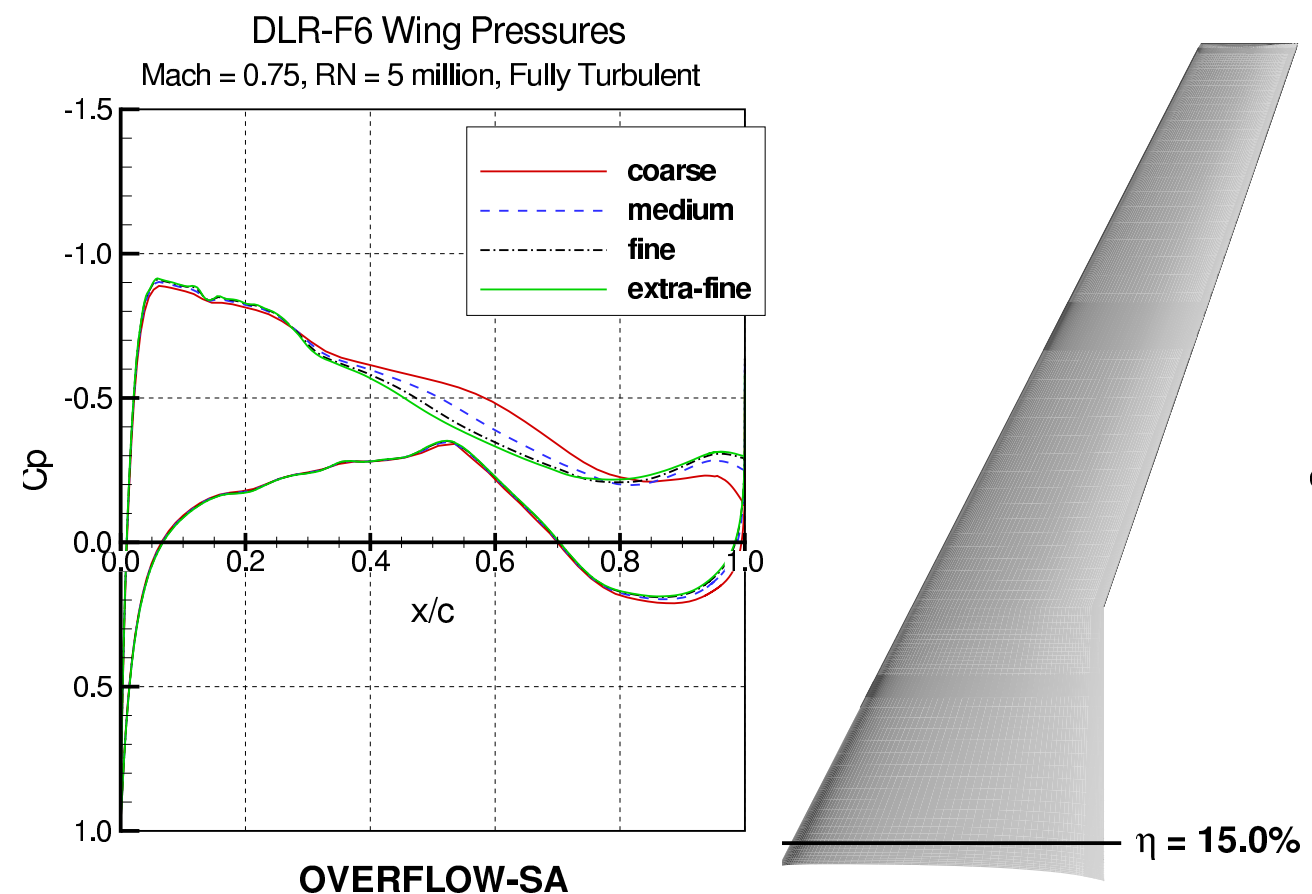

DLR-F6 Wing Pressures Mach $=0.75, \mathrm{RN}=5$ million, Fully Turbulent

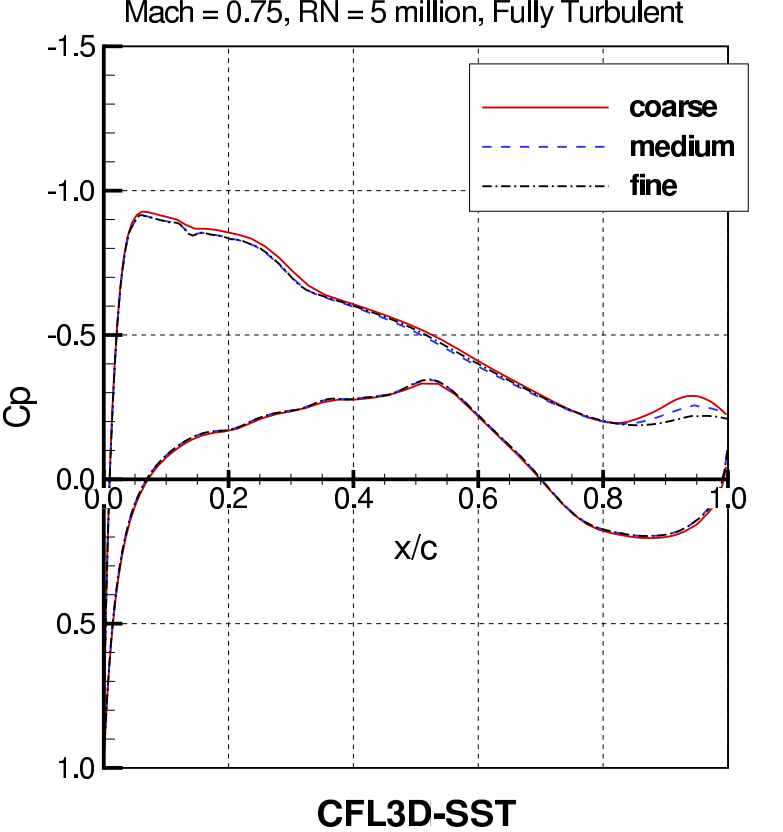

Figure 24. DLR-F6 Wing Pressure Comparison at .150 Semispan. 

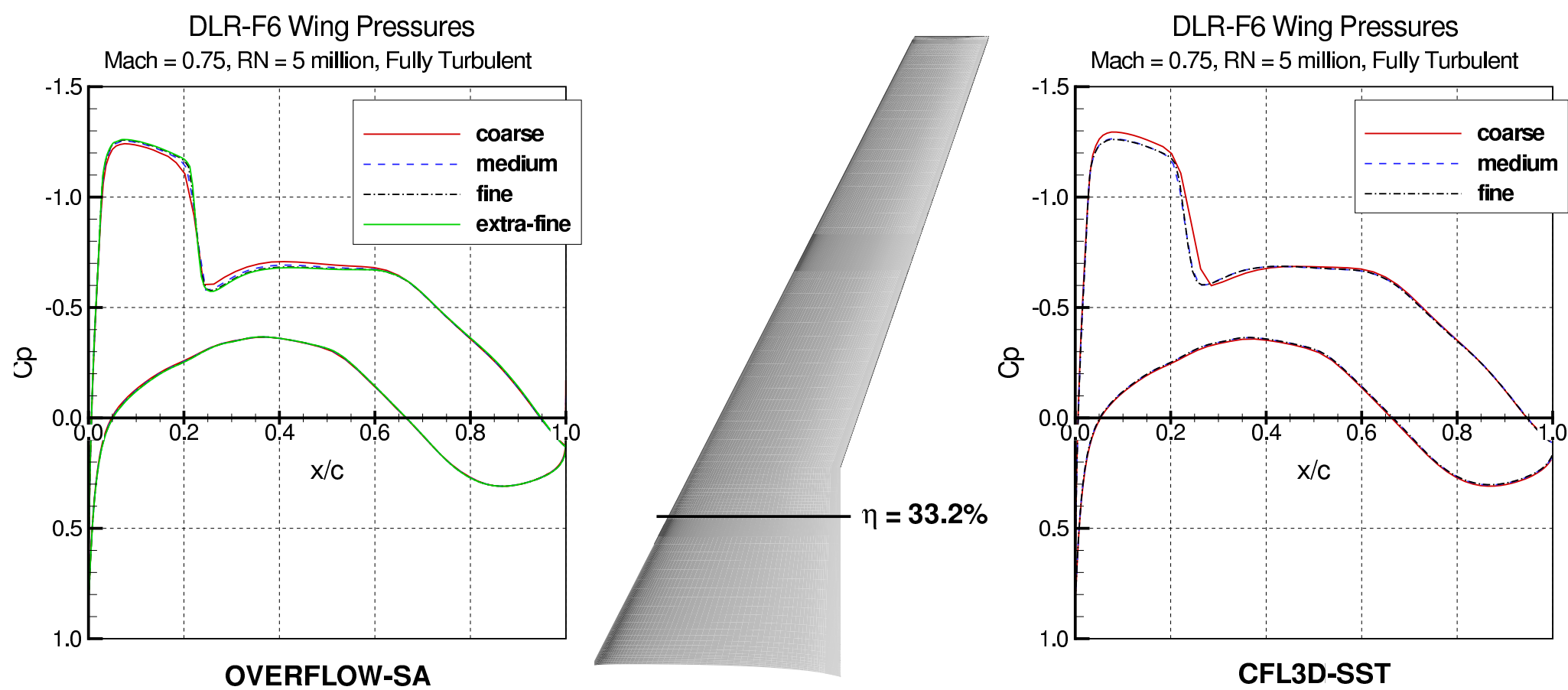

Figure 25. DLR-F6 Wing Pressure Comparison at .332 Semispan.
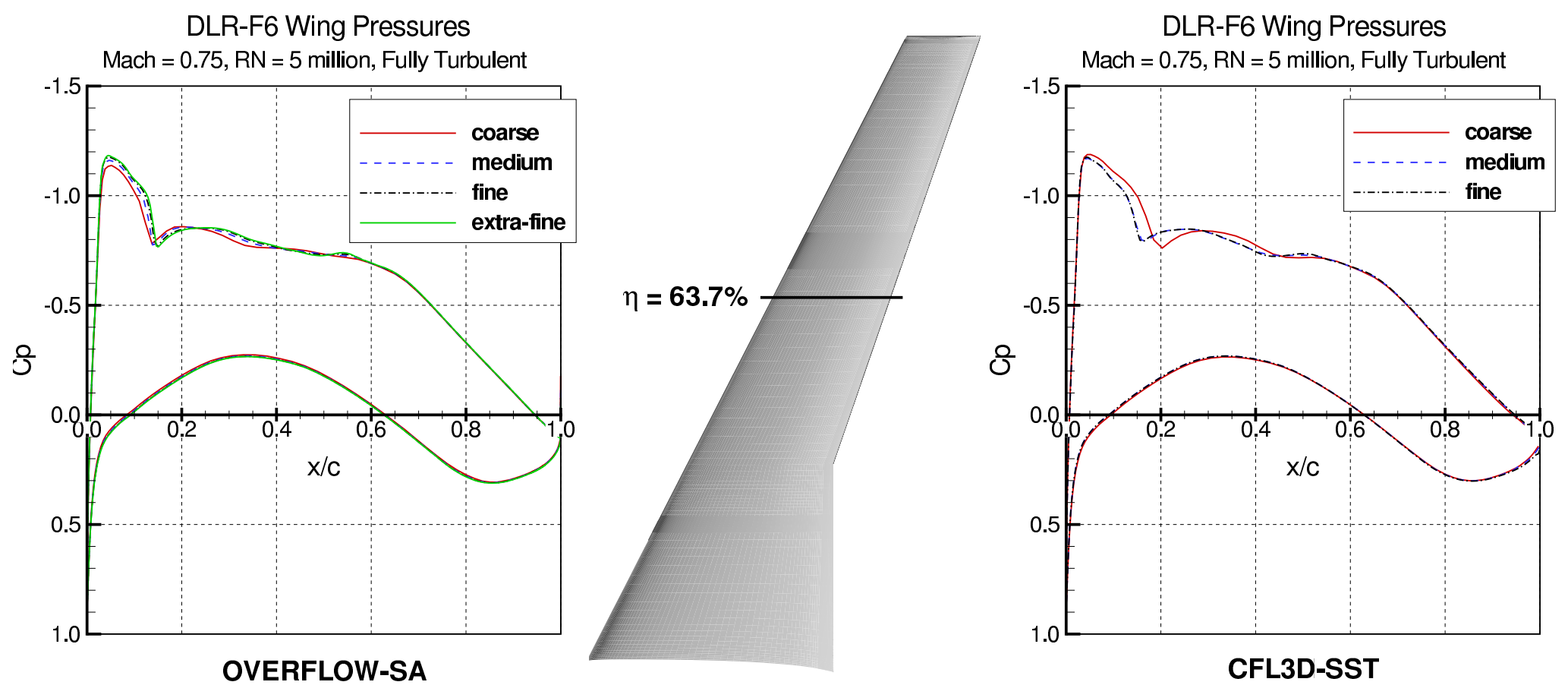

Figure 26. DLR-F6 Wing Pressure Comparison at .637 Semispan. 


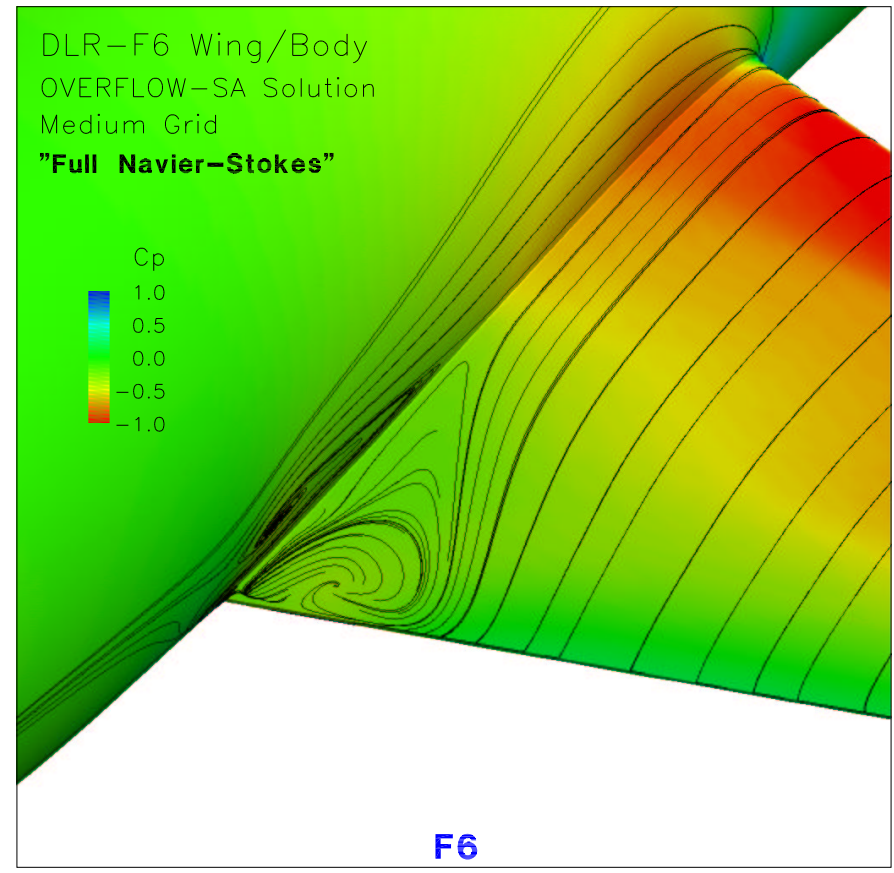

(a) F6 Results.

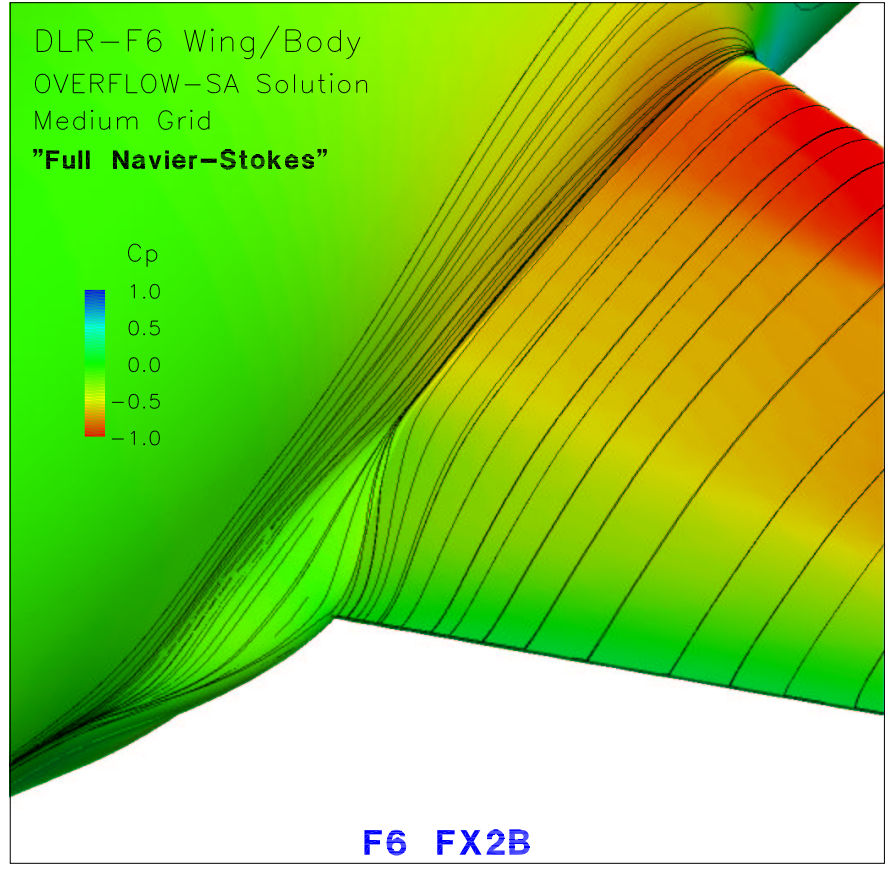

(b) FX2B Results.

Figure 27. DLR-F6 OVERFLOW-SA Surface Streamline Comparison: Wing/Body Isometric View.

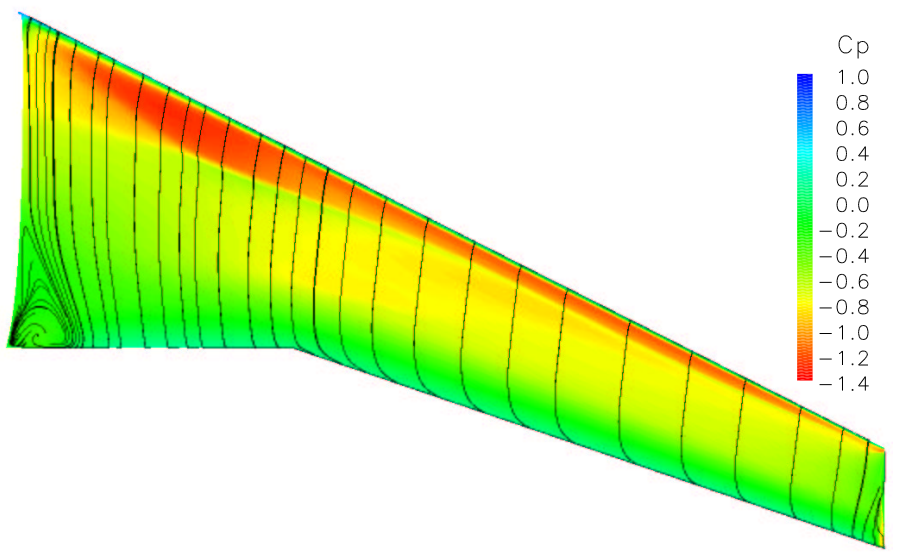

(a) F6 Results.

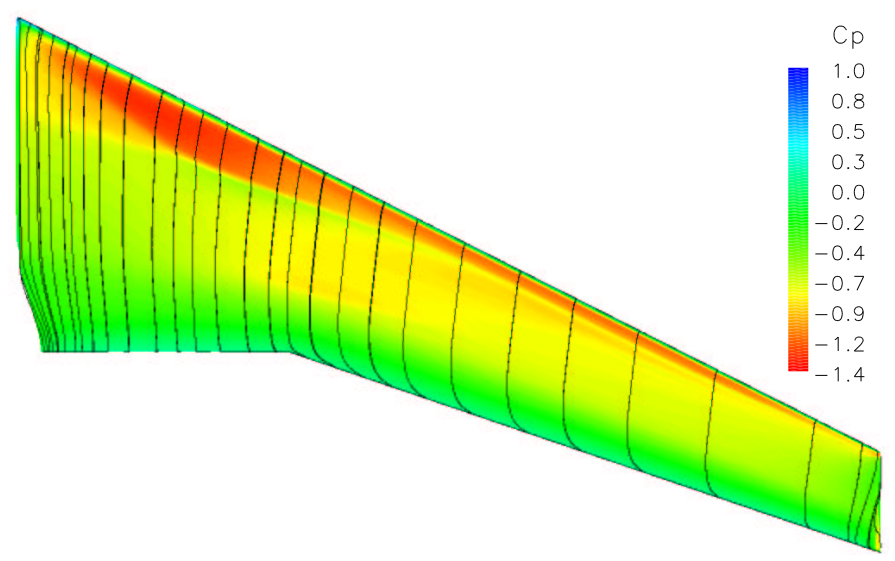

(b) FX2B Results. 
DLR-F6 Wing/Body Results: F6 Drag Polar

Mach $=0.75, R_{N}=5.0$ million, Fully Turbulent, Medium Grid

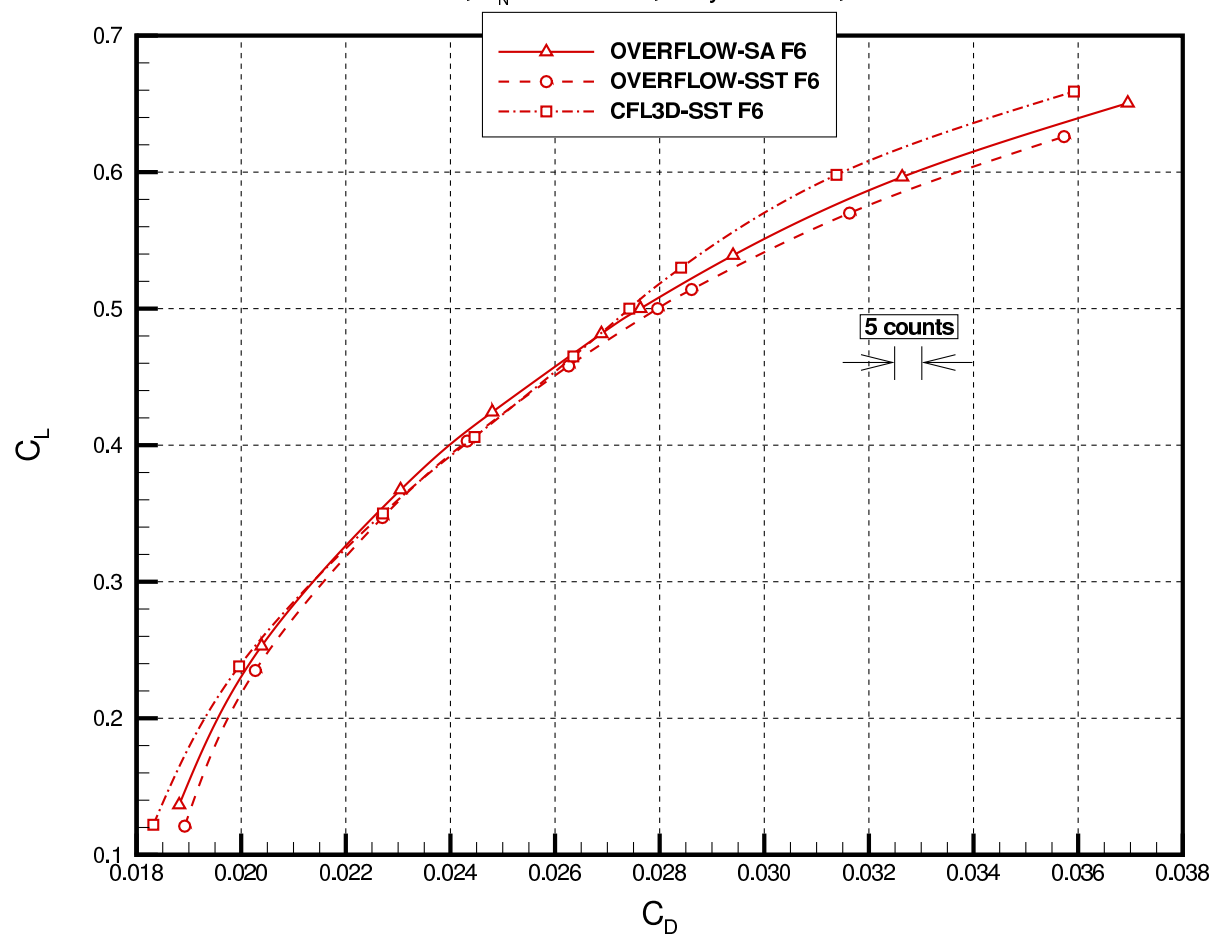

Figure 29. DLR-F6 Drag Polar: Baseline F6.

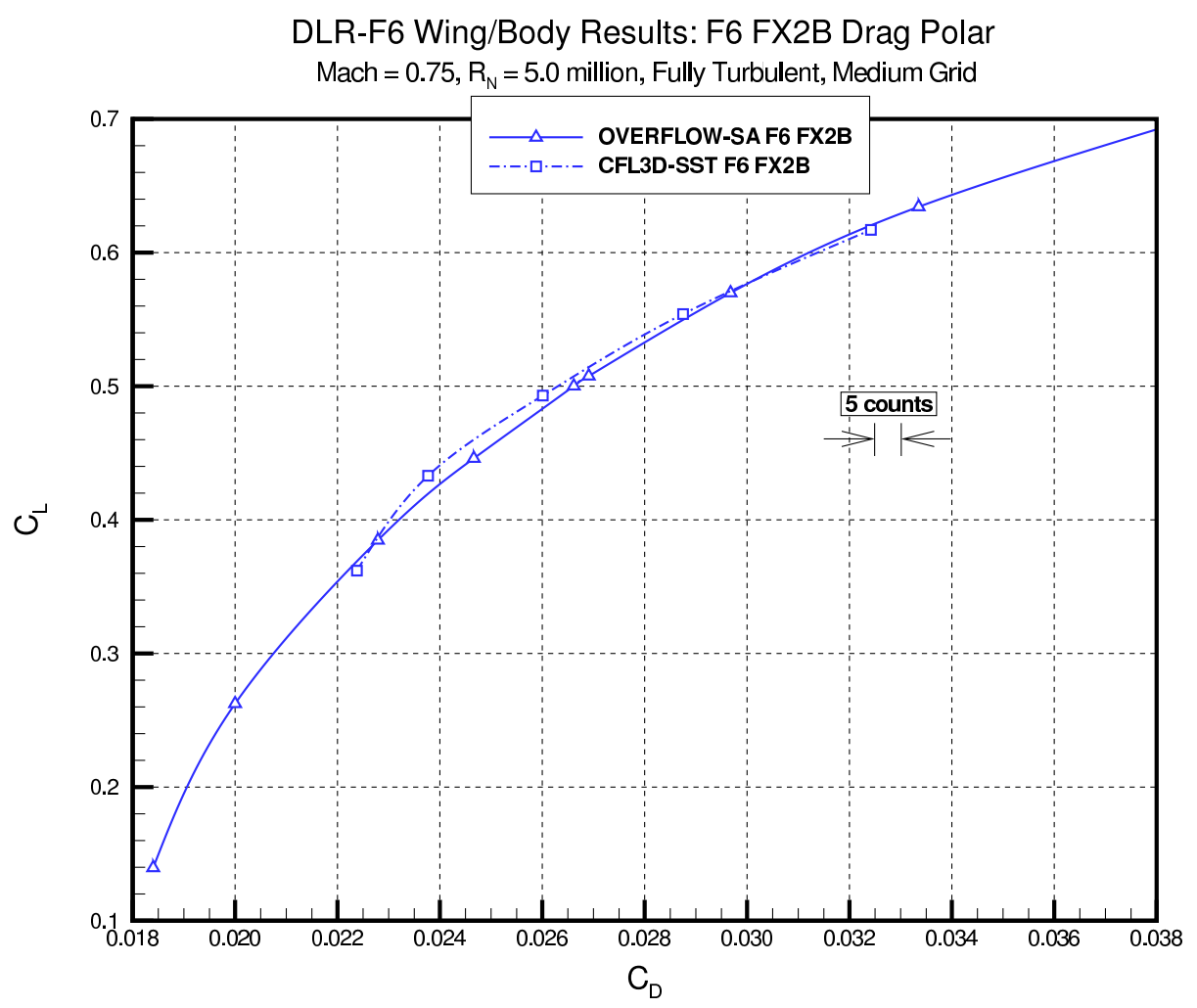

Figure 30. DLR-F6 Drag Polar: F6 FX2B. 


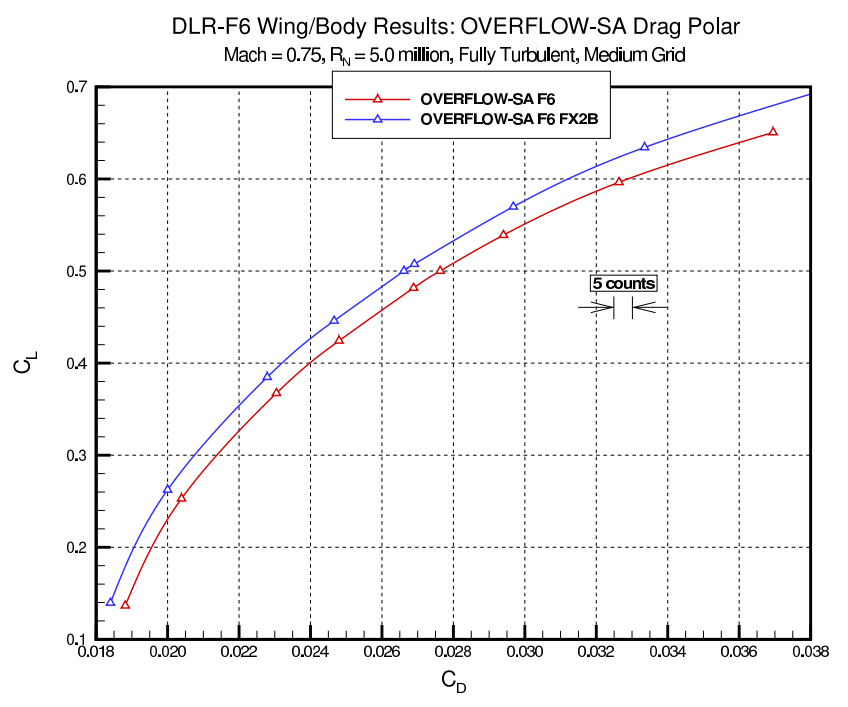

(a) OVERFLOW Results.

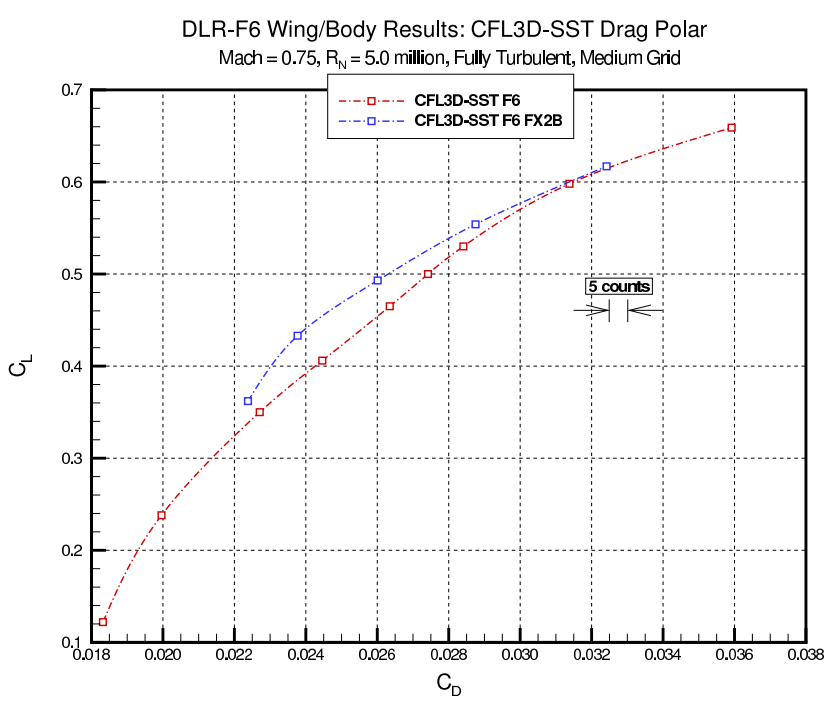

(b) CFL3D Results.

Figure 31. DLR-F6 Drag Polars.

DLR-F6 Wing/Body Results: F6 $C_{L}$ vs $\alpha$

Mach $=0.75, R_{N}=5.0$ million, Fully Turbulent, Medium Grid

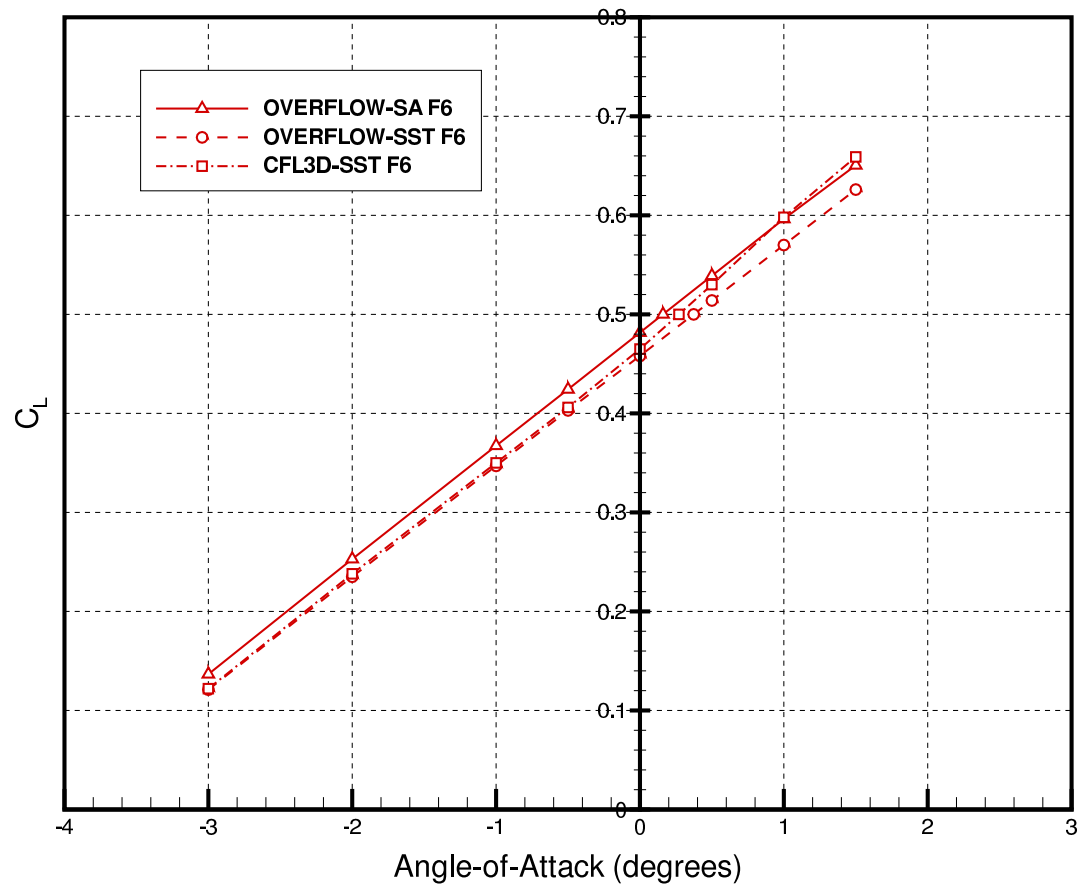

Figure 32. DLR-F6 Lift Curve: Baseline F6. 


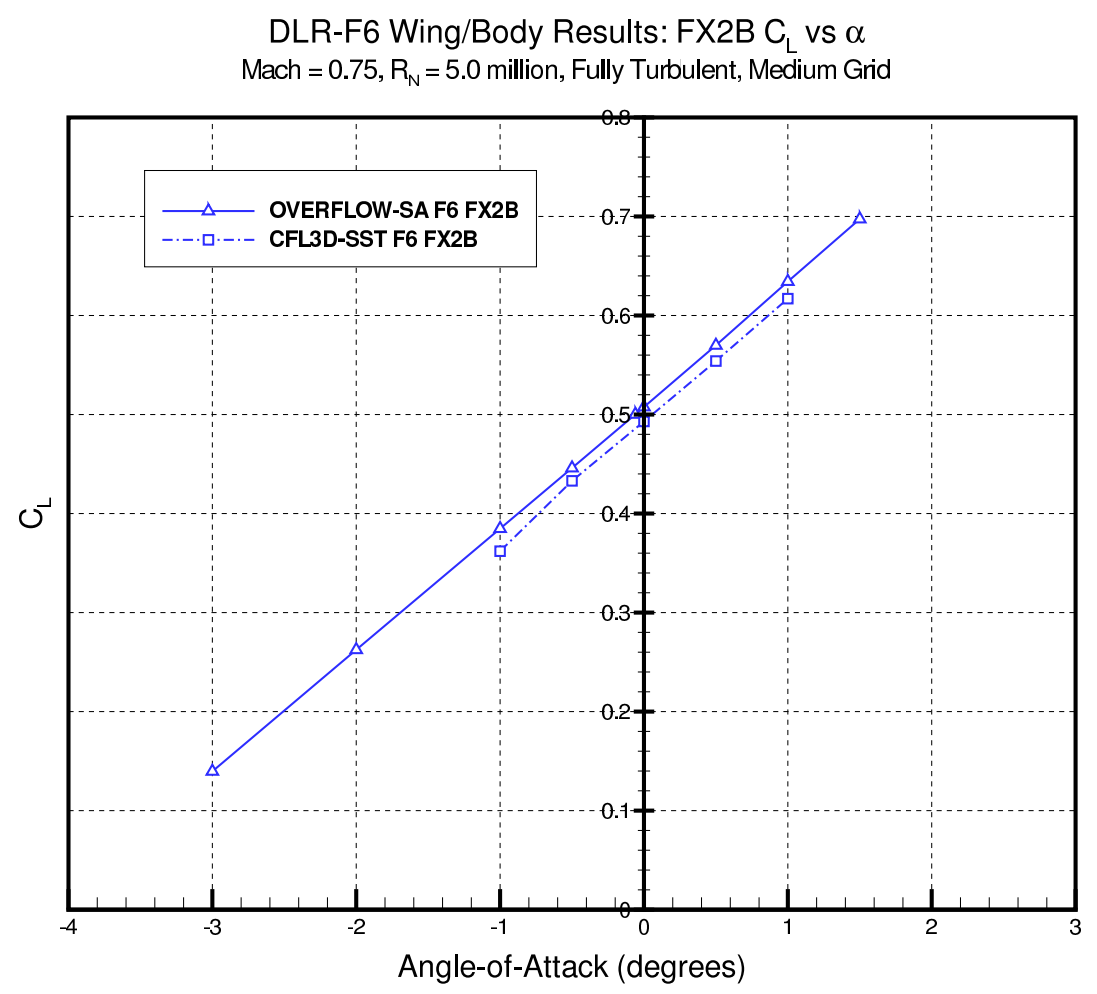

Figure 33. DLR-F6 Lift Curve: F6 FX2B.

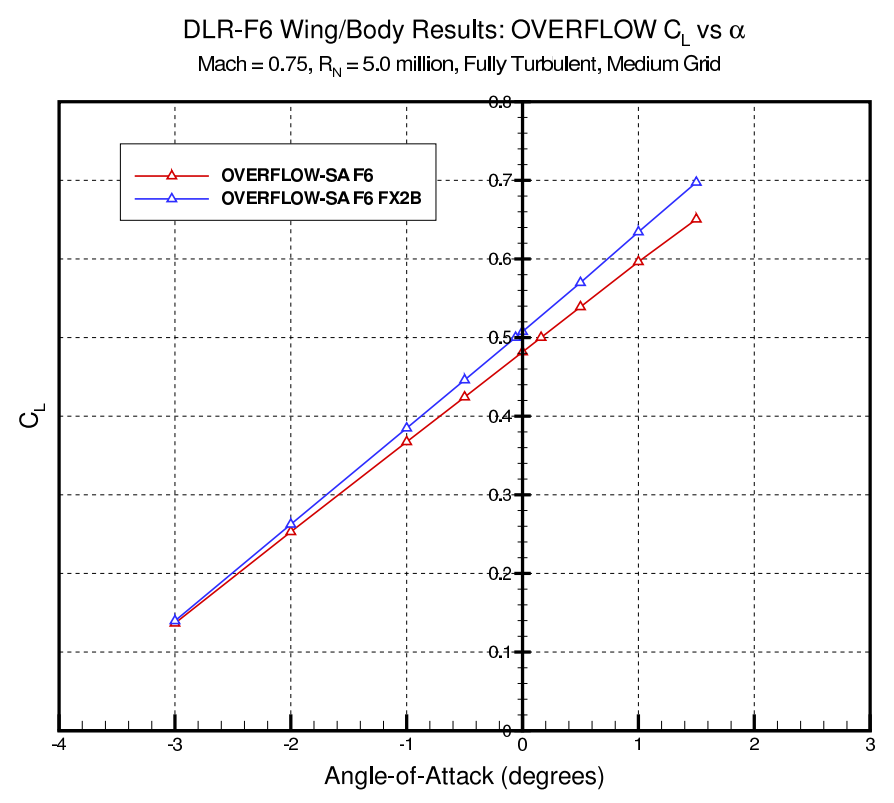

(a) OVERFLOW Results.

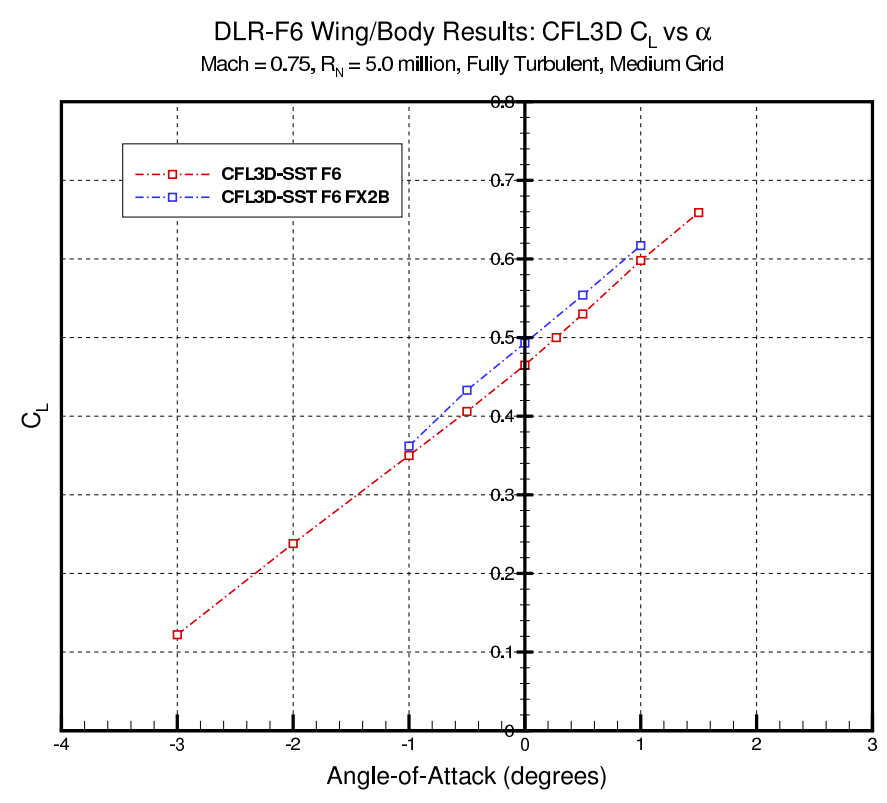

(b) CFL3D Results.

Figure 34. DLR-F6 Lift Curves. 
DLR-F6 Wing/Body Results: F6 $\mathrm{C}_{\mathrm{L}}$ vs $\mathrm{C}_{\mathrm{M}}$ Mach $=0.75, R_{N}=5.0$ million, Fully Turbulent, Medium Grid

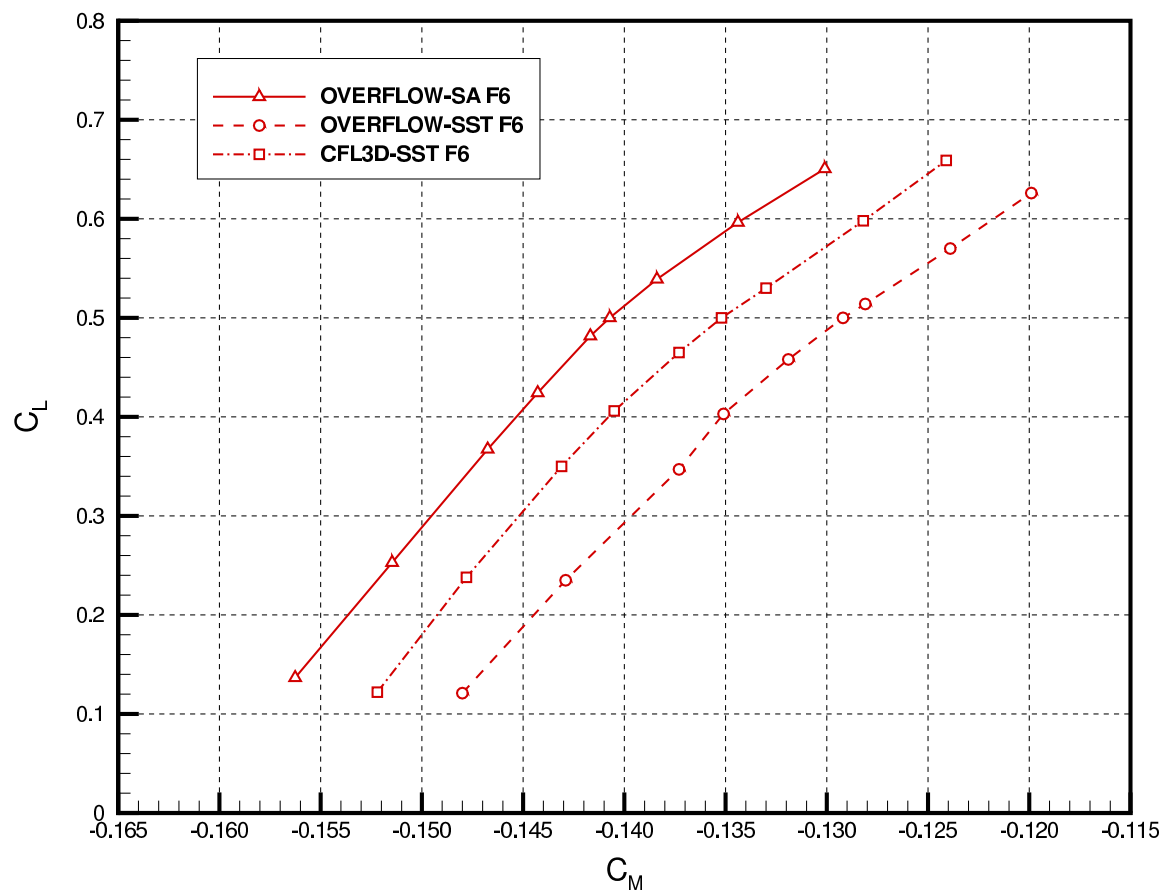

Figure 35. DLR-F6 Pitching Moment: Baseline F6.

DLR-F6 Wing/Body Results: FX2B $C_{L}$ vs $C_{M}$ Mach $=0.75, R_{N}=5.0$ million, Fully Turbulent, Medium Grid

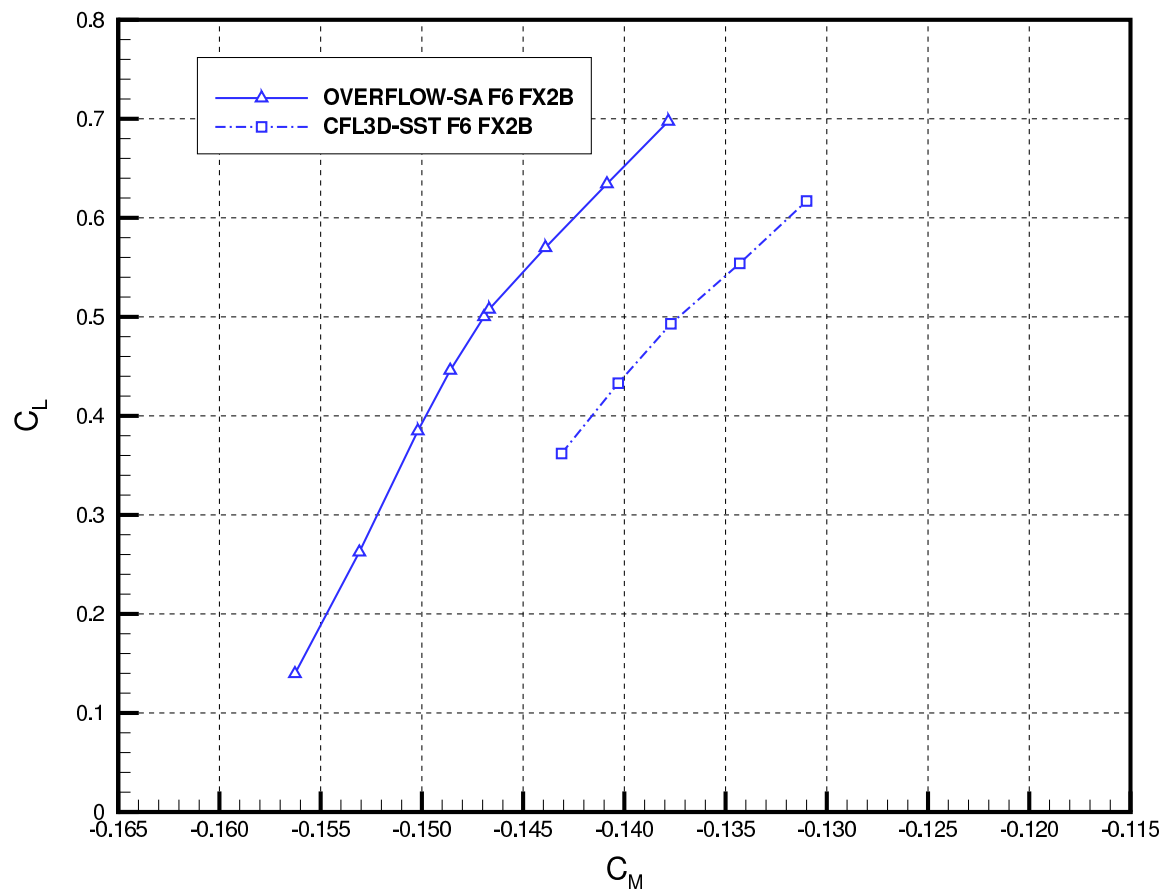

Figure 36. DLR-F6 Pitching Moment: F6 FX2B. 


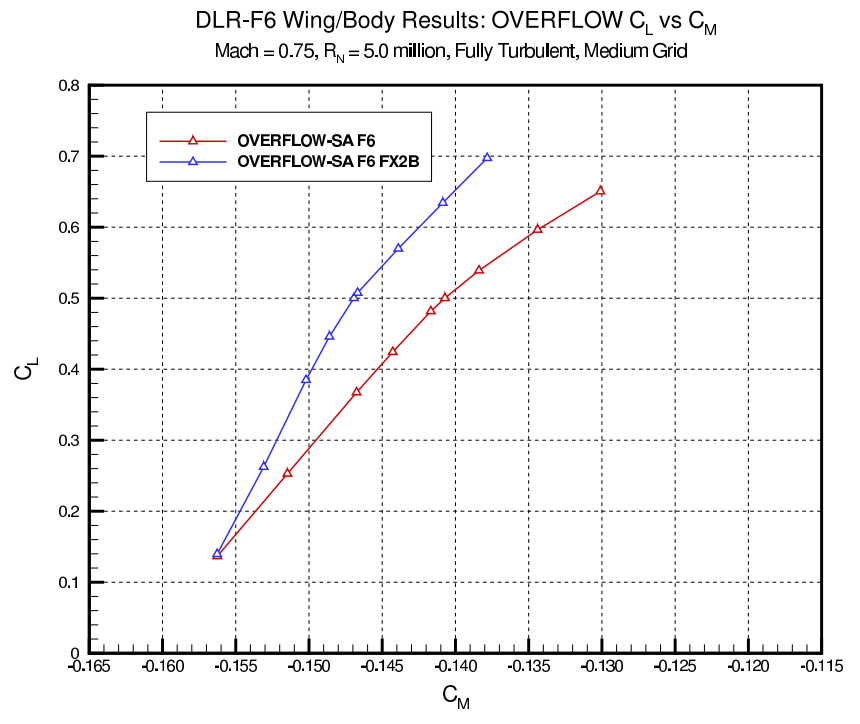

(a) OVERFLOW Results.

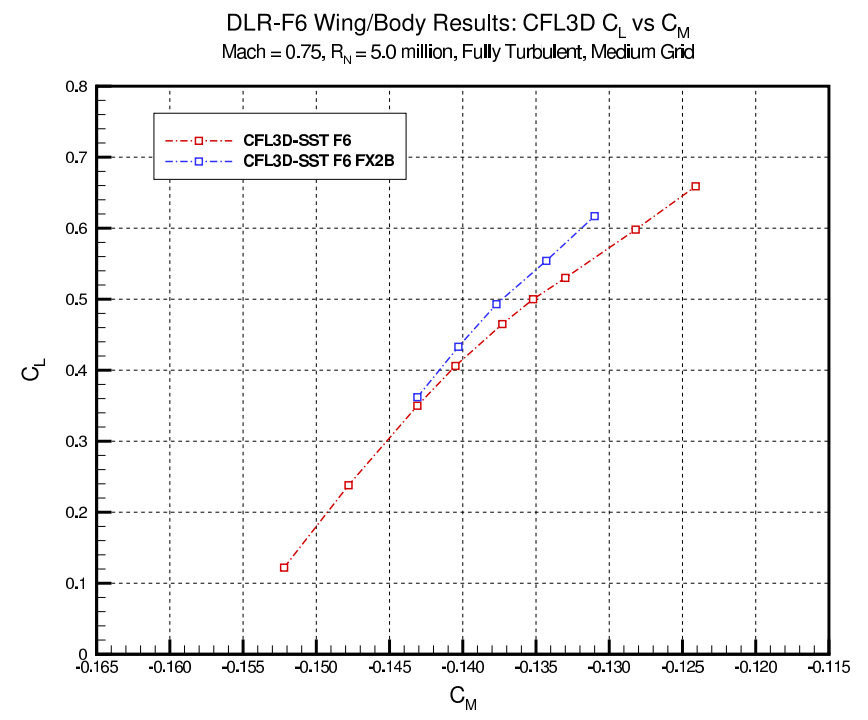

(b) CFL3D Results.

Figure 37. DLR-F6 Pitching Moment.

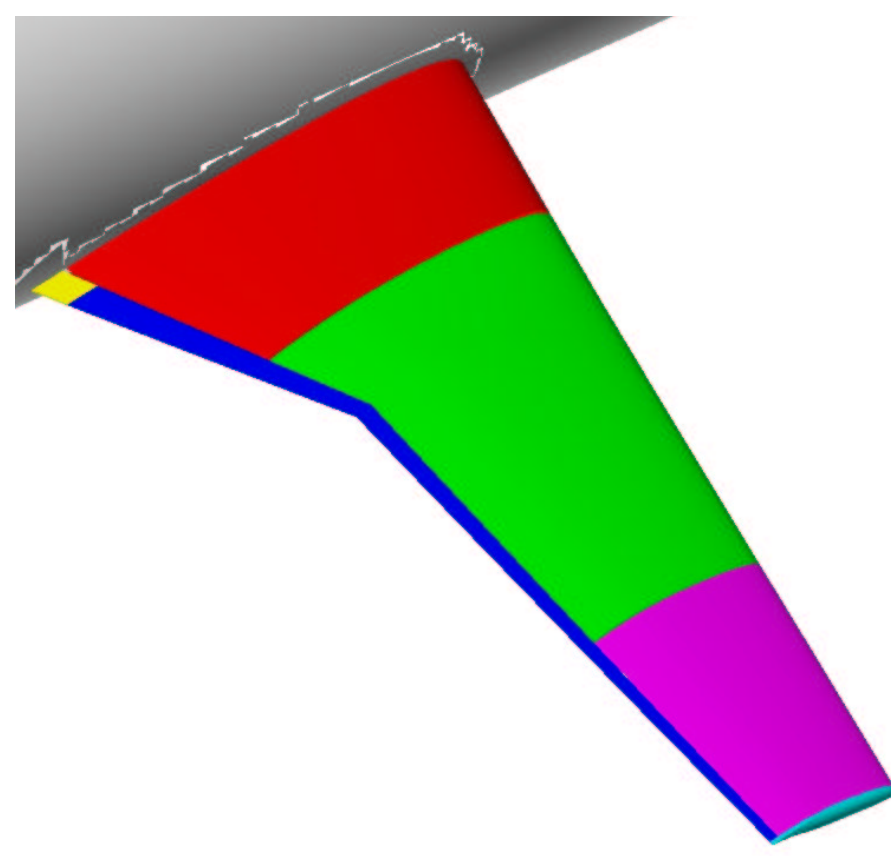

(a) Wing Isometric View.

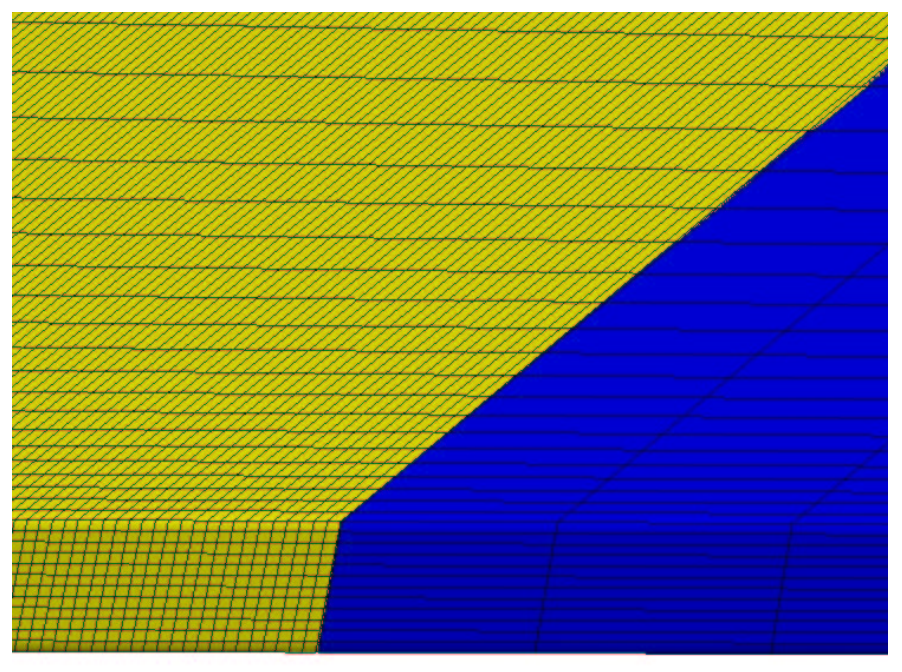

(b) Trailing-Edge Looking Forward.

Figure 38. DLR-F6 Dense Trailing-Edge Cap Grid Study. 


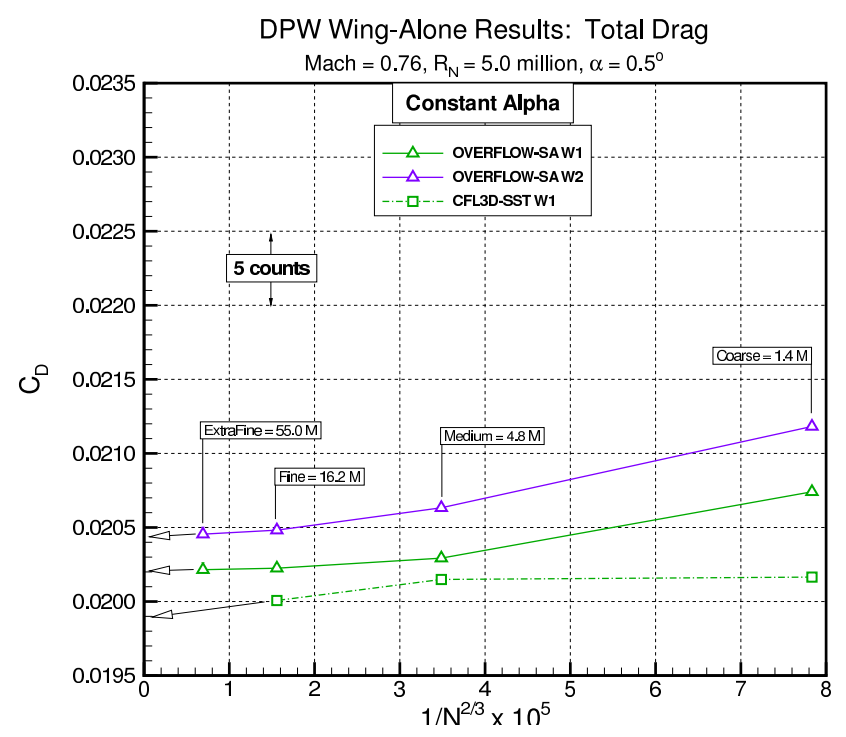

(a) Constant $\alpha$ Results.

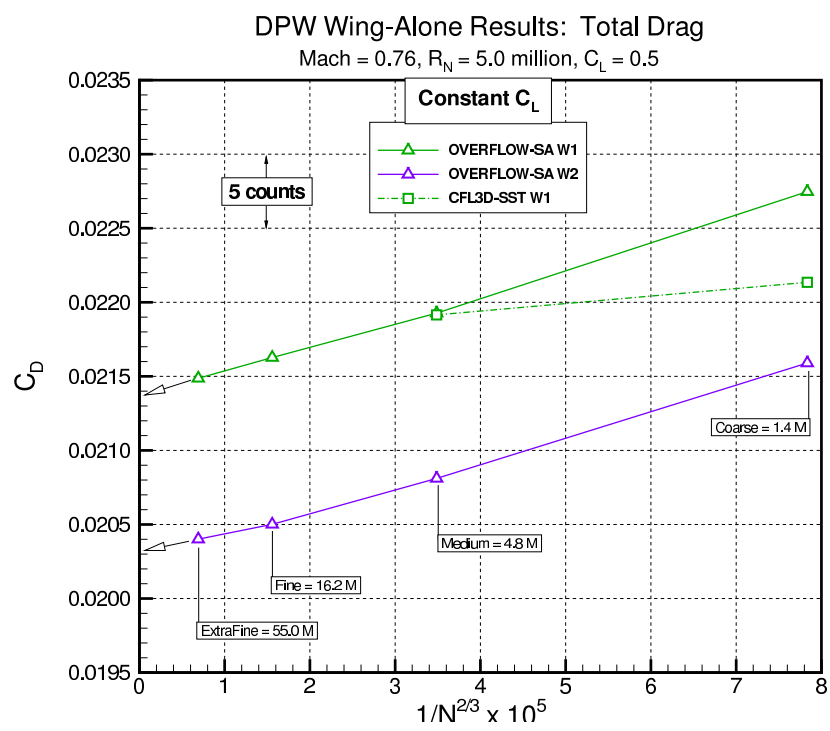

(b) Constant $C_{L}$ Results.

Figure 39. DPW-W1/W2 Grid Convergence Study: Total Drag.

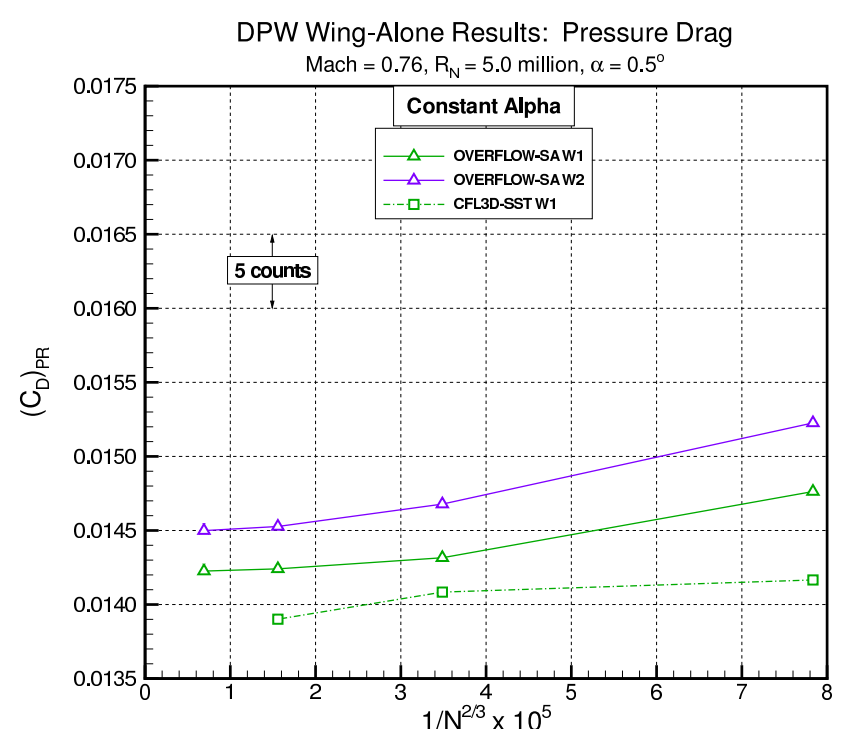

(a) Constant $\alpha$ Results.

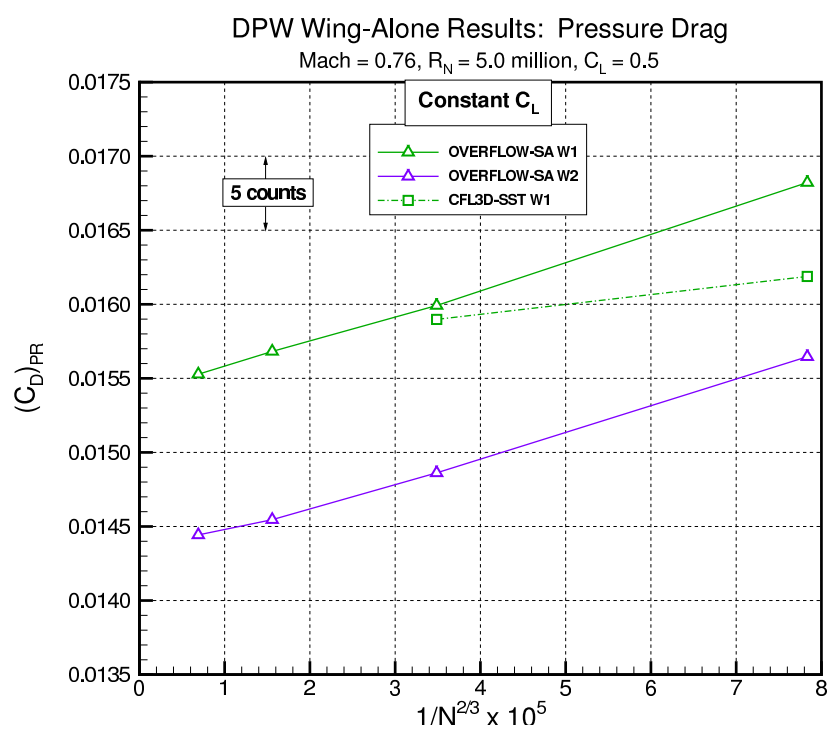

(b) Constant $C_{L}$ Results.

Figure 40. DPW-W1/W2 Grid Convergence Study: Pressure Drag. 


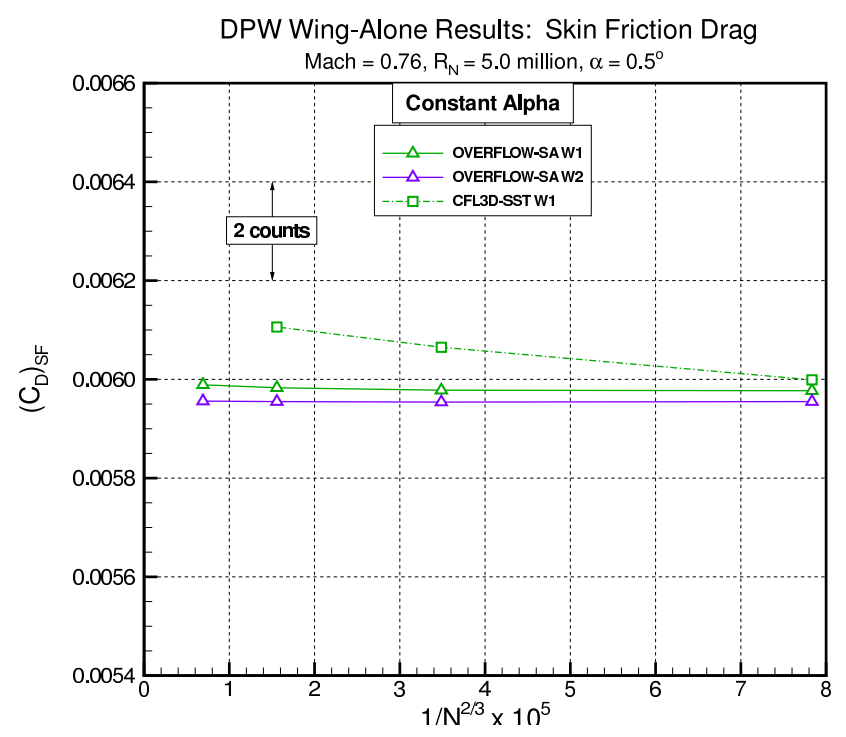

(a) Constant $\alpha$ Results.

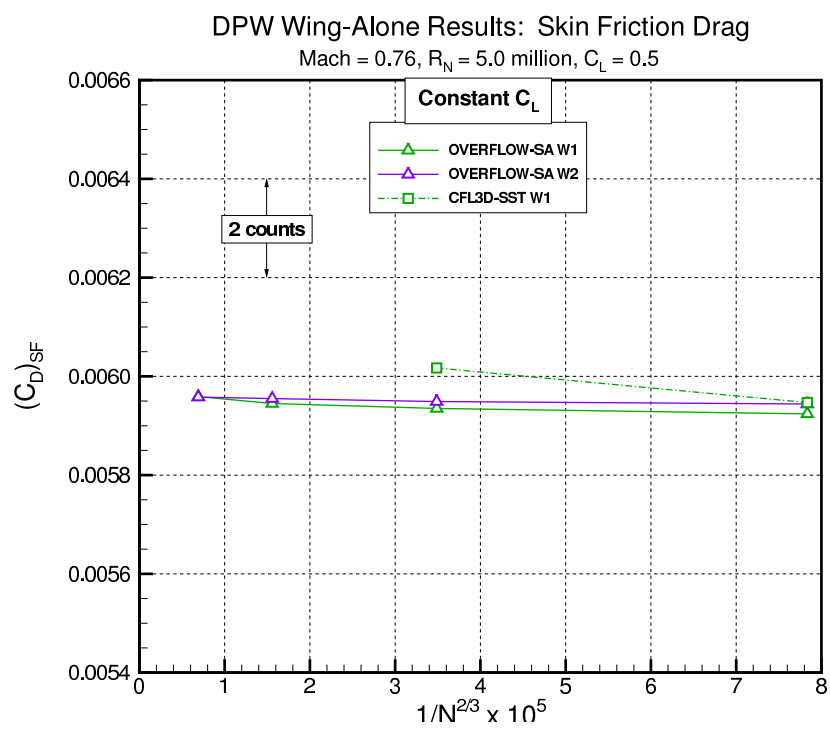

(b) Constant $C_{L}$ Results.

Figure 41. DPW-W1/W2 Grid Convergence Study: Skin Friction Drag.

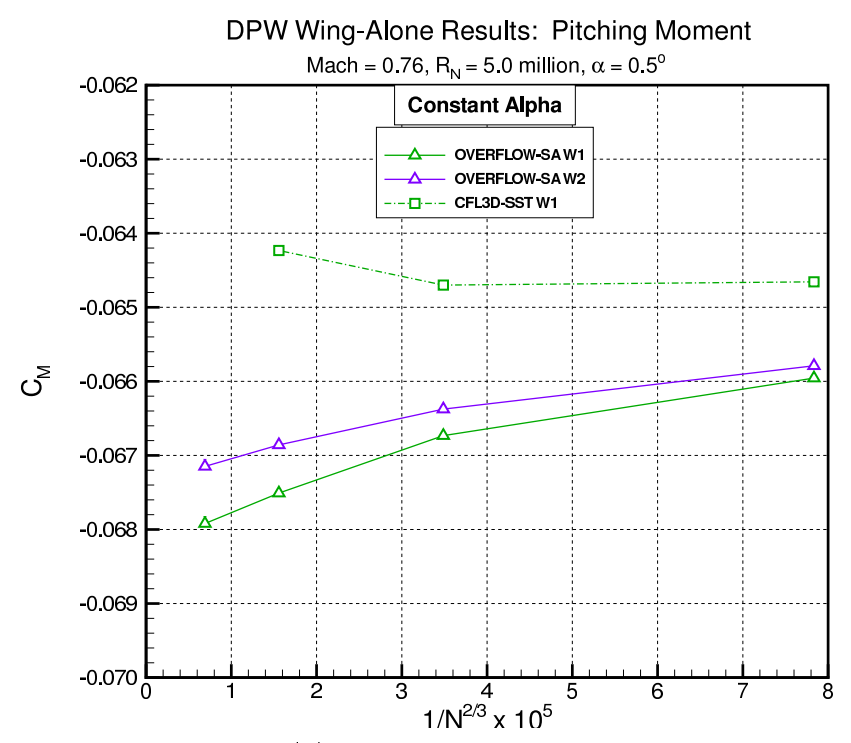

(a) Constant $\alpha$ Results.

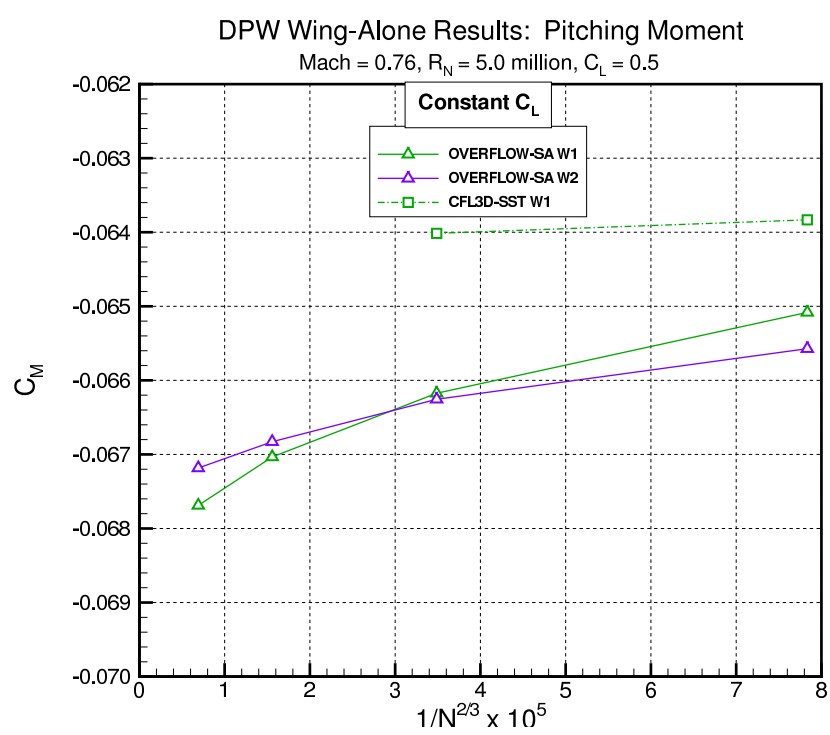

(b) Constant $C_{L}$ Results.

Figure 42. DPW-W1/W2 Grid Convergence Study: Pitching Moment. 


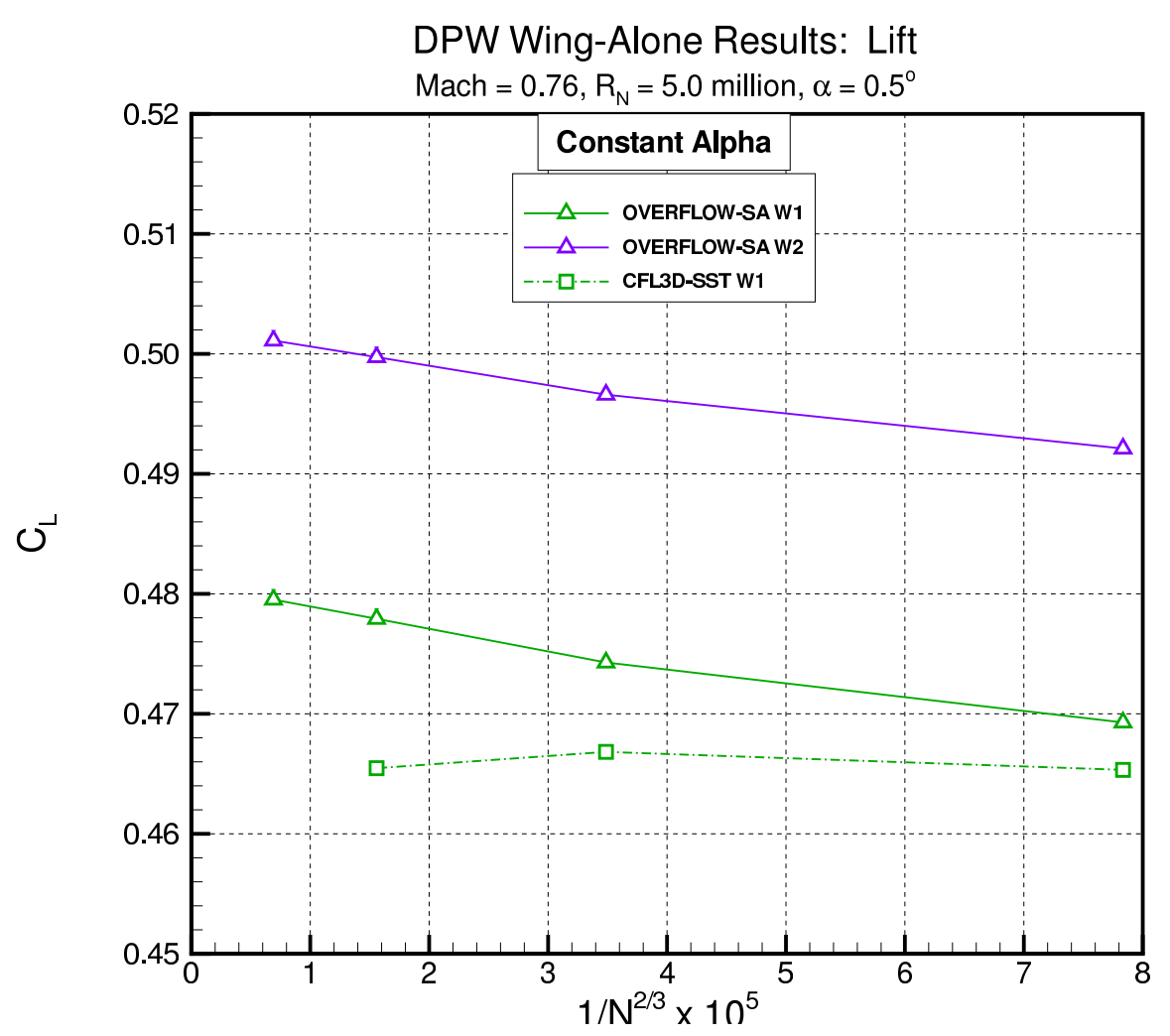

Figure 43. DPW-W1/W2 Grid Convergence Study: Lift at Constant Angle-of-Attack.

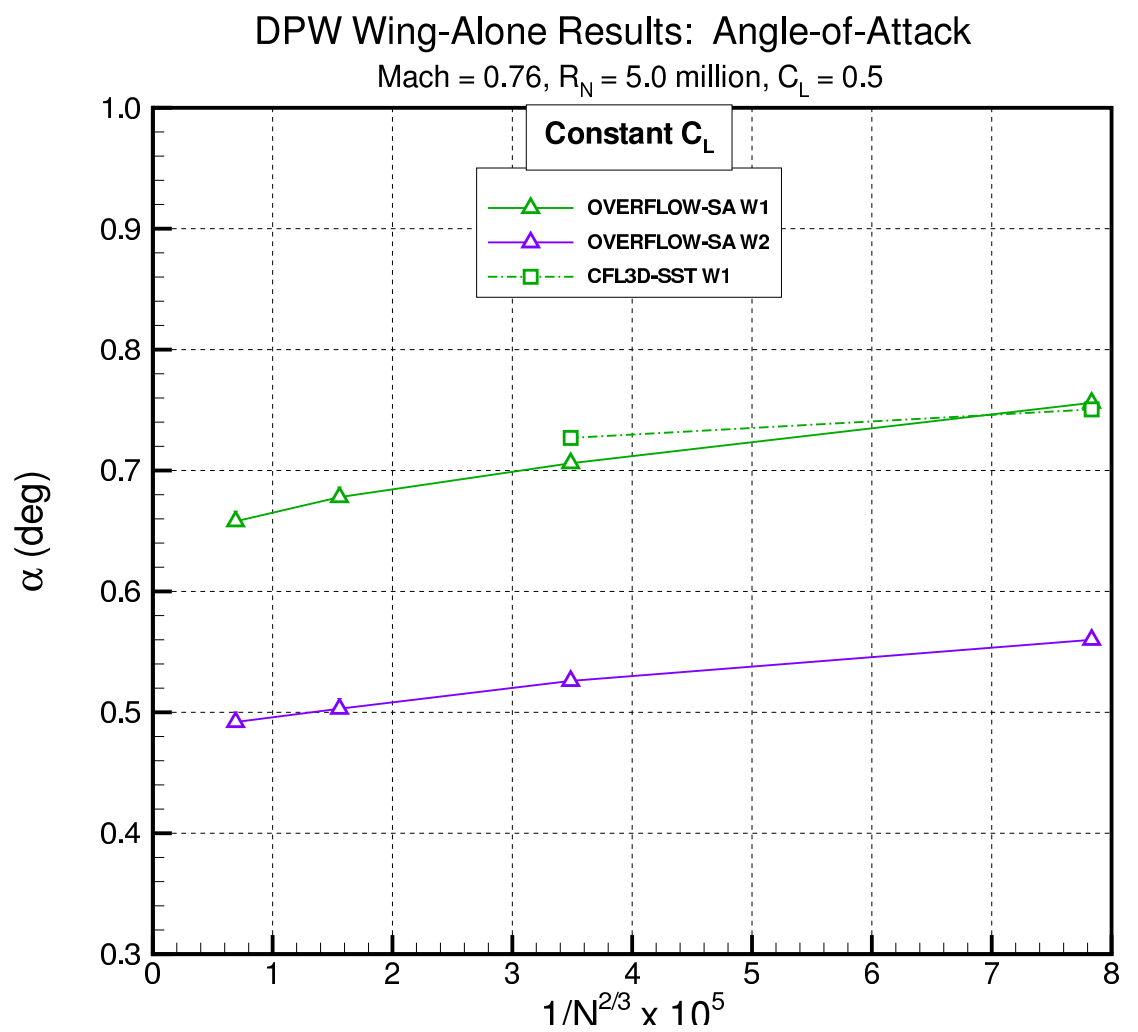

Figure 44. DPW-W1/W2 Grid Convergence Study: Angle-of-Attack at Constant Lift. 


\section{DPW-W1 Wing Pressures}

Mach $=0.76, \mathrm{RN}=5$ million, $\mathrm{C}_{\mathrm{L}}=0.5$, Fully Turbulent

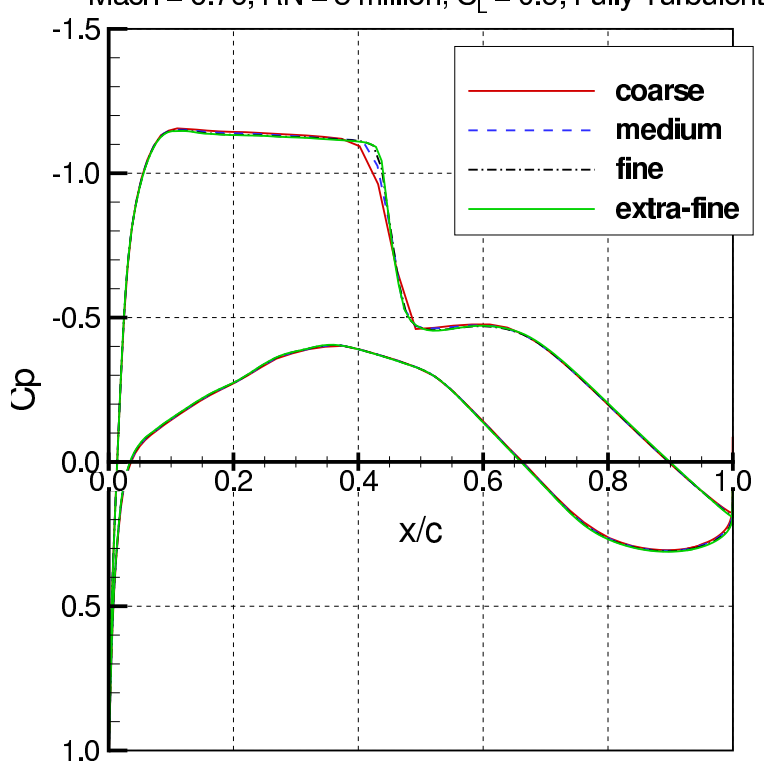

OVERFLOW-SA

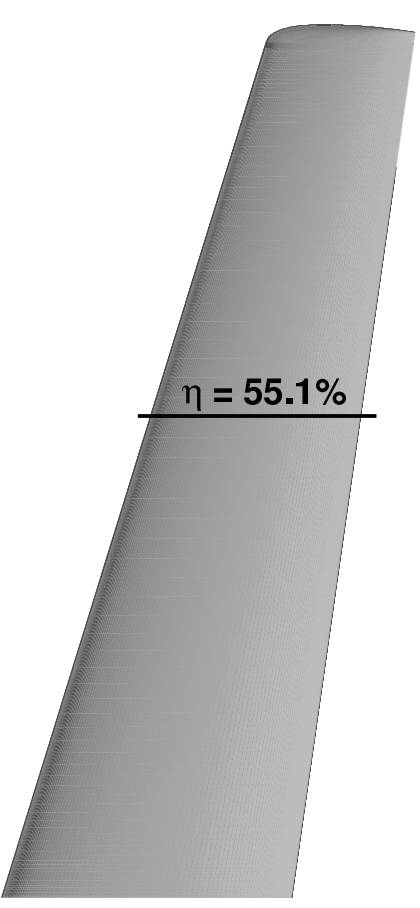

DPW-W1 Wing Pressures

Mach $=0.76, R N=5$ million, $C_{L}=0.5$, Fully Turbulent

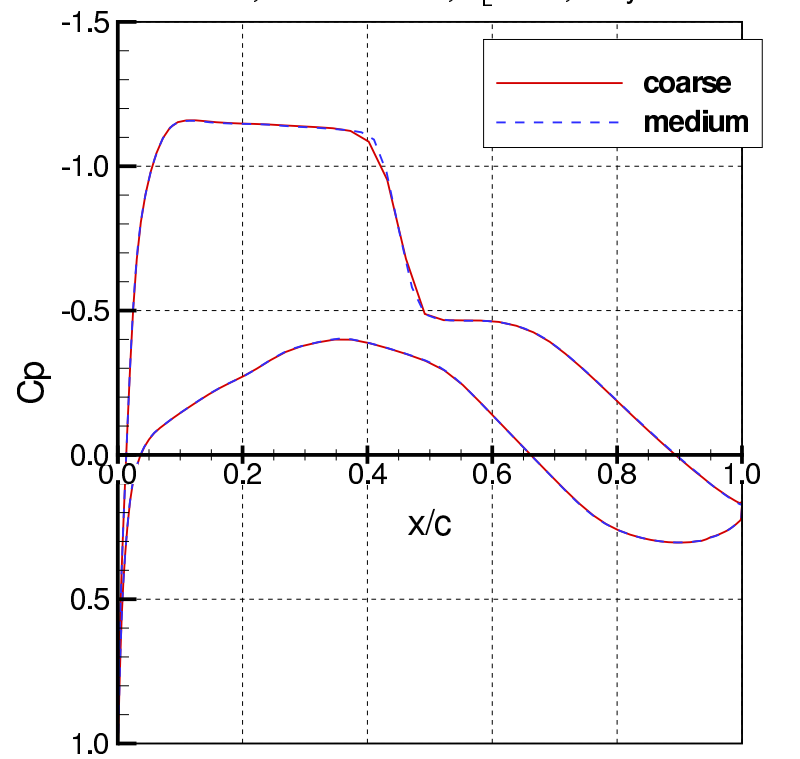

CFL3D-SST

Figure 45. DPW-W1 Wing Pressure Comparison at .551 Semispan.

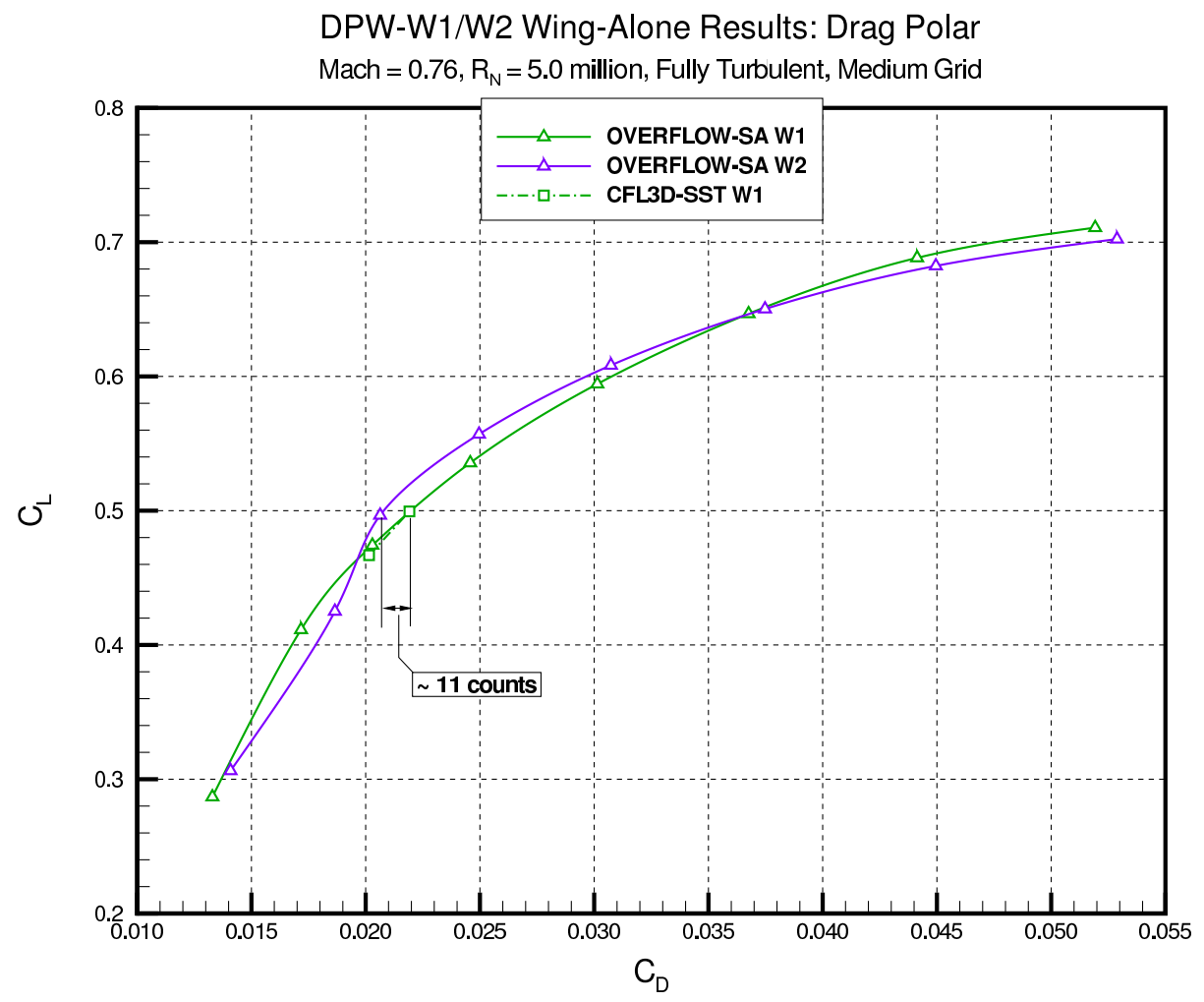

Figure 46. DPW-W1/W2 Drag Polars. 


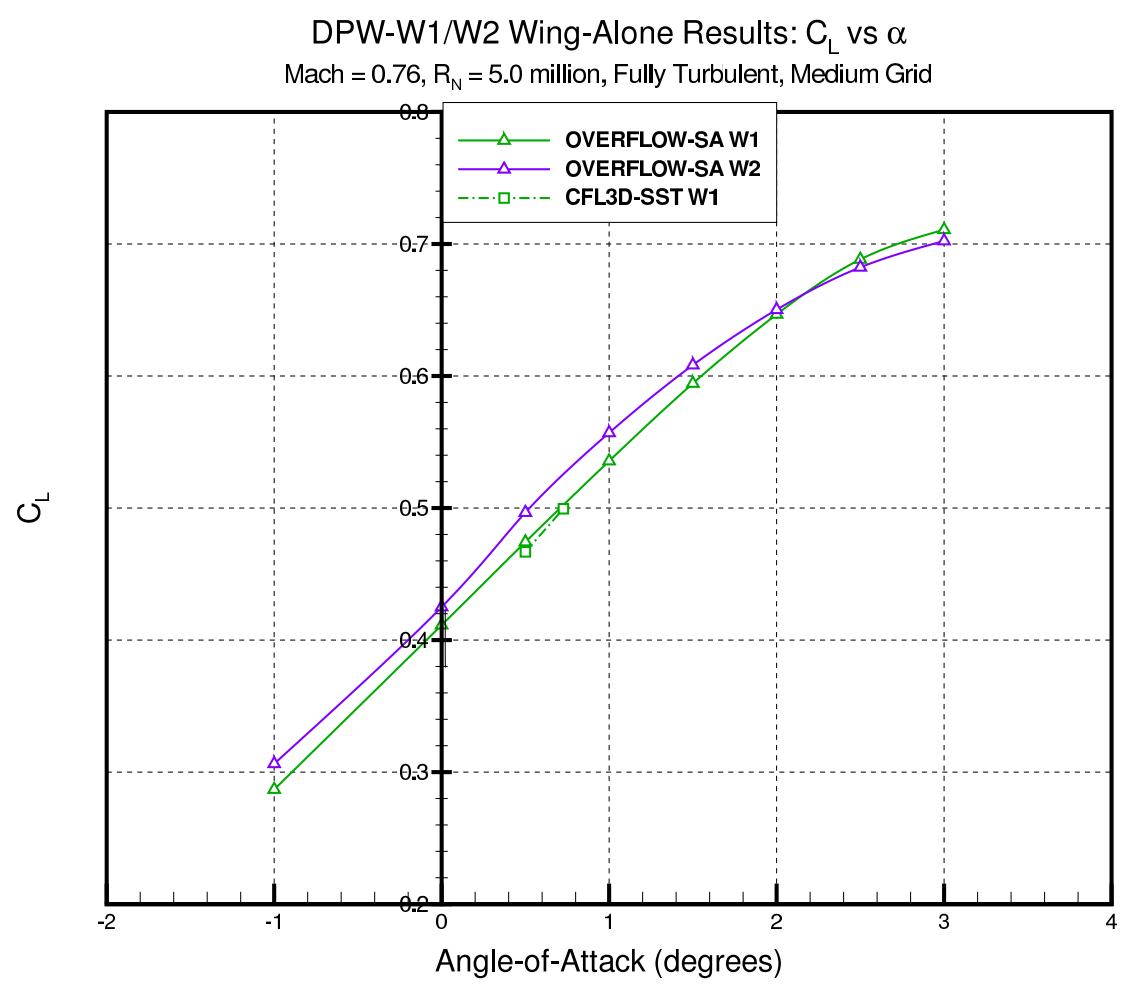

Figure 47. DPW-W1/W2 Lift Curves.

DPW-W1/W2 Wing-Alone Results: $C_{L}$ vs $C_{M}$ Mach $=0.76, R_{N}=5.0$ million, Fully Turbulent, Medium Grid

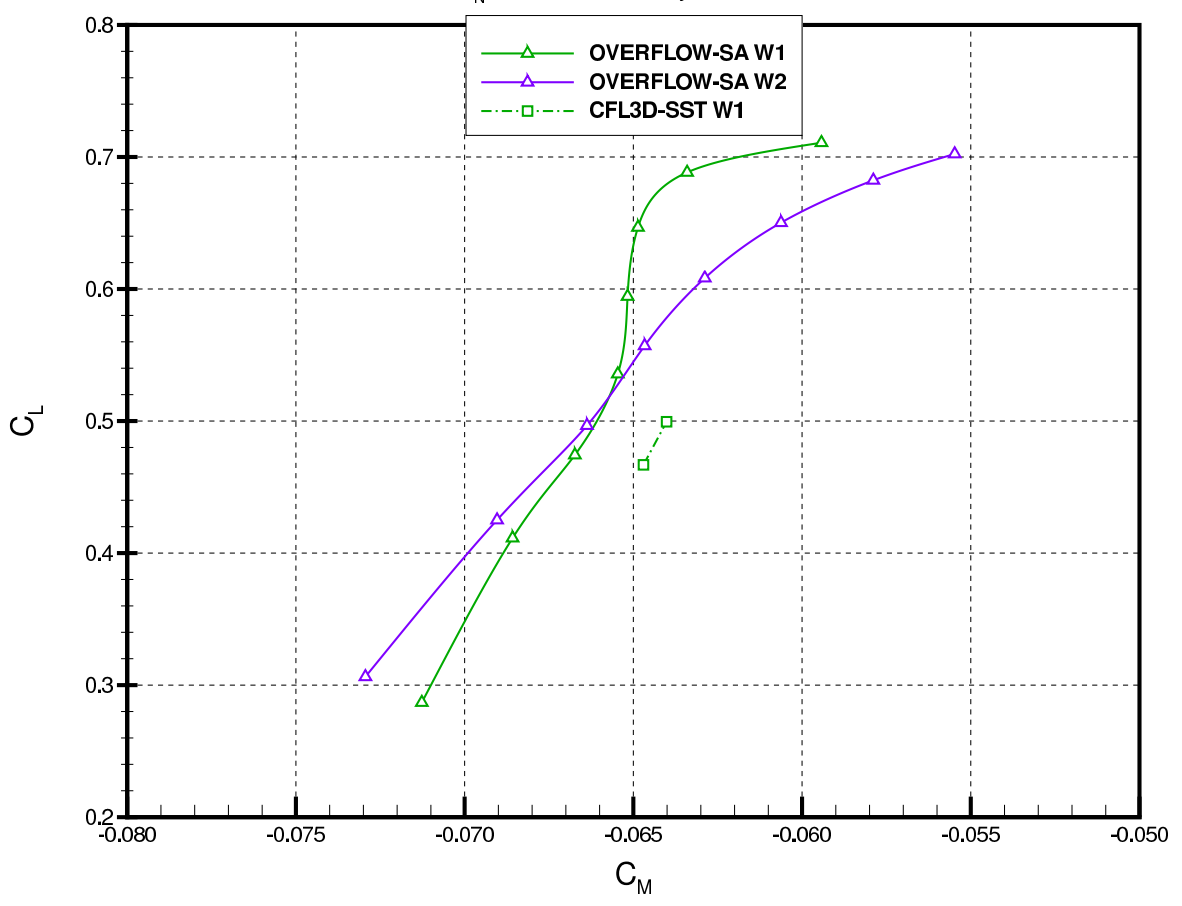

Figure 48. DPW-W1/W2 Pitching Moment. 

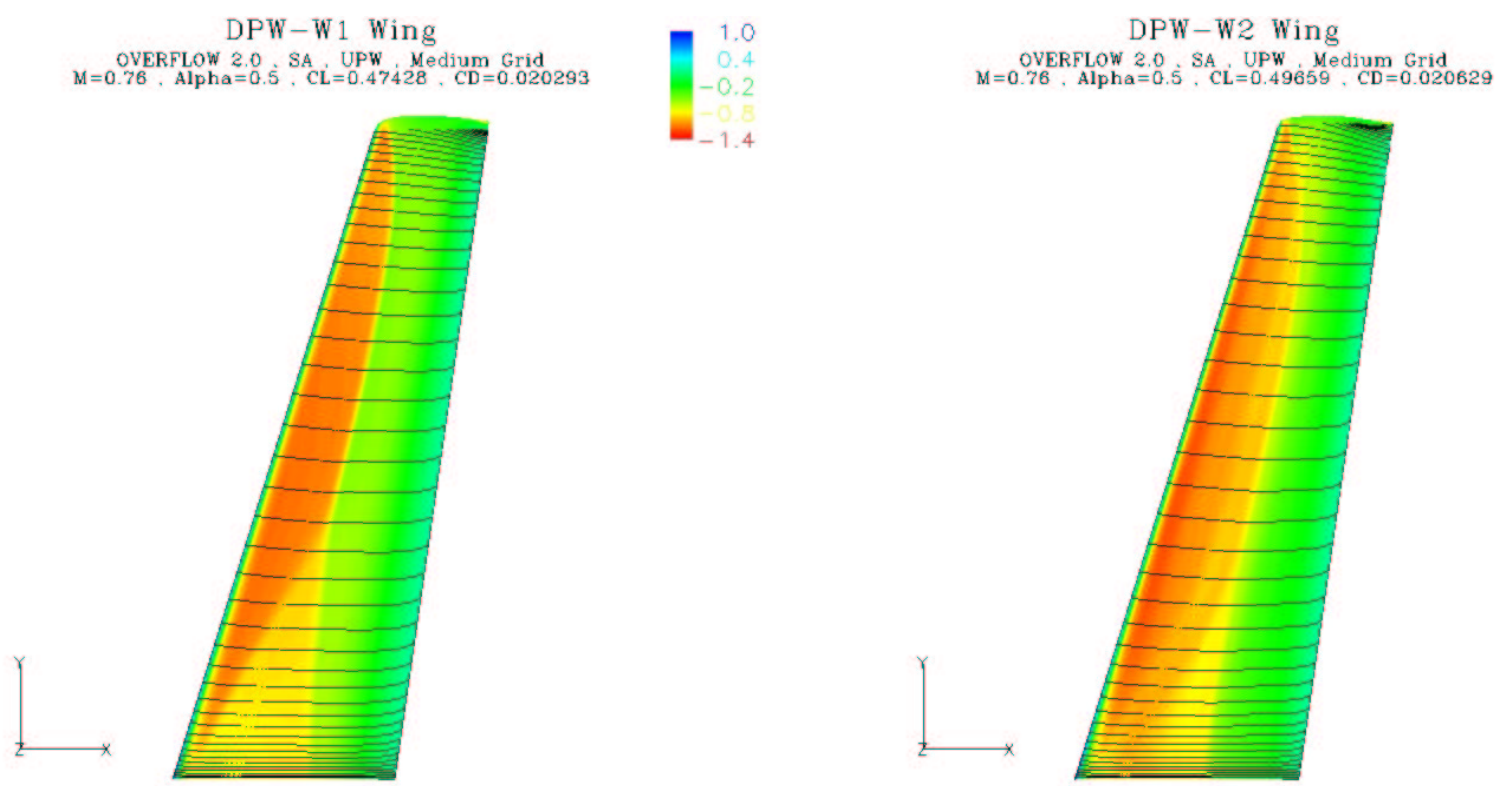

Figure 49. DPW-W1/W2 Surface Streamline Comparison at $\alpha=0.5^{\circ}$.
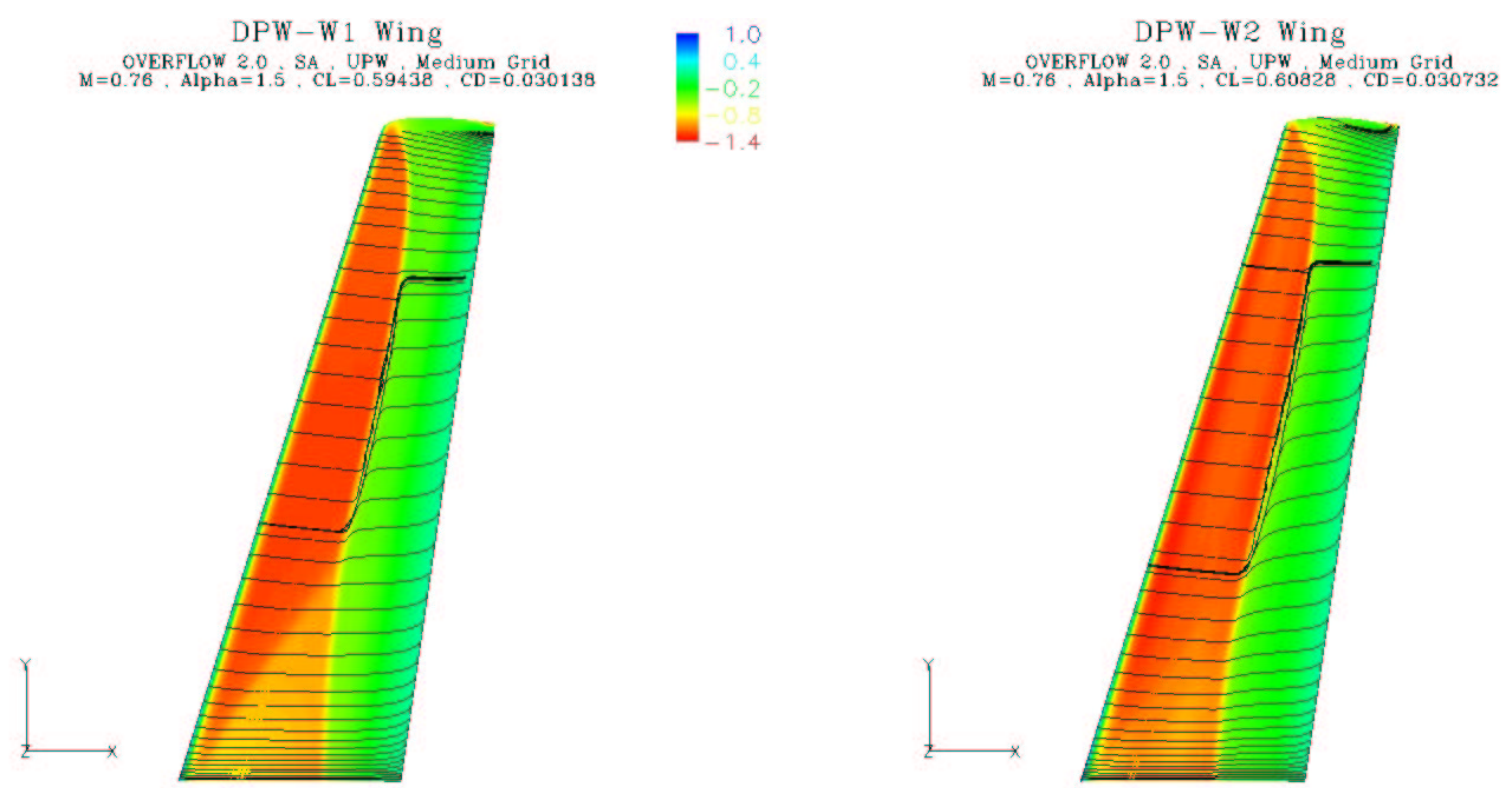

Figure 50. DPW-W1/W2 Surface Streamline Comparison at $\alpha=1.5^{\circ}$. 

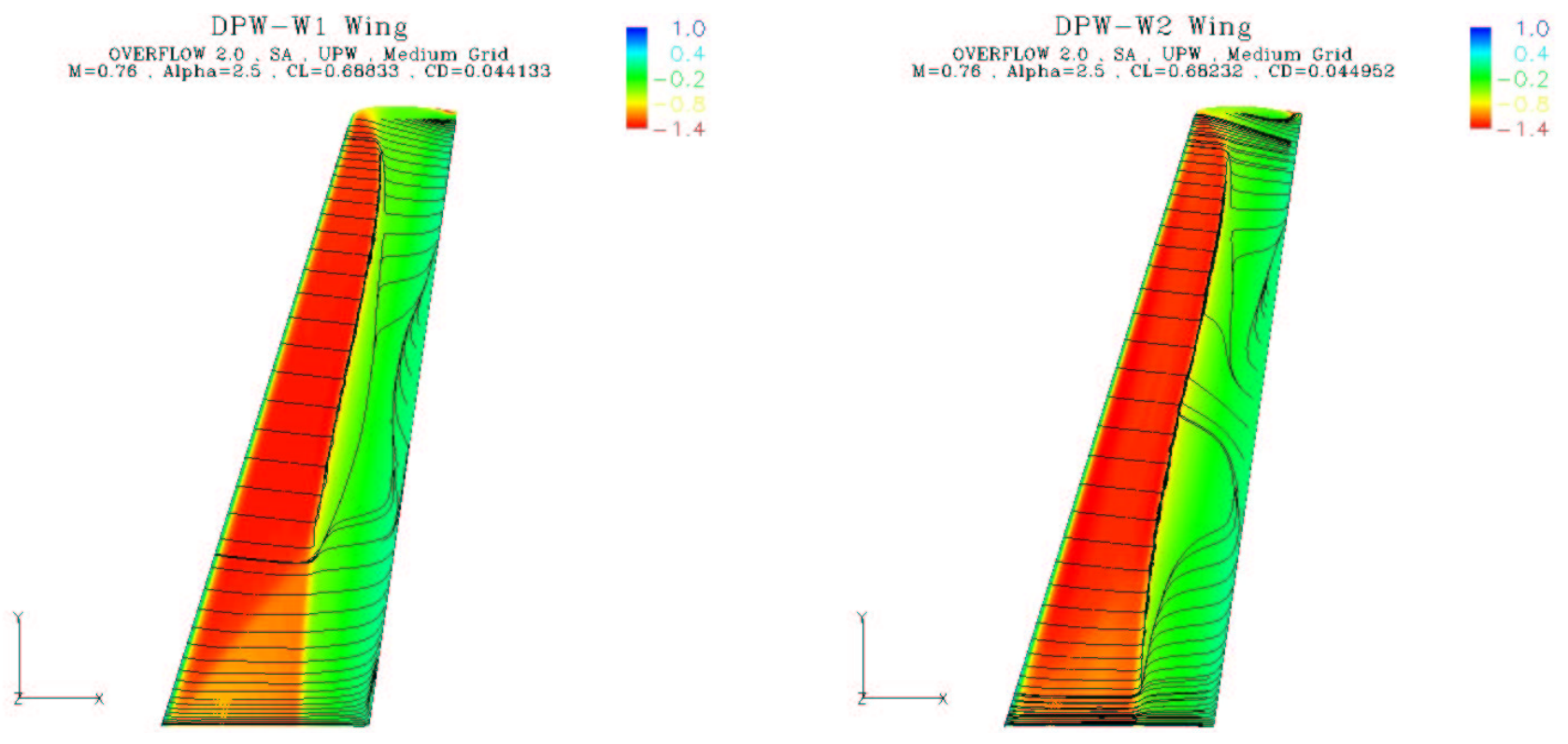

Figure 51. DPW-W1/W2 Surface Streamline Comparison at $\alpha=2.5^{\circ}$. 